Arch. Rational Mech. Anal. manuscript No.

(will be inserted by the editor)

\title{
The Positive Solutions of the Matukuma Equation and the Problem of Finite Radius and Finite Mass
}

JÜRGEN BATT \& YI LI

\begin{abstract}
This work is an extensive study of the 3 different types of positive solutions of the Matukuma equation $\frac{1}{r^{2}}\left(r^{2} \phi^{\prime}\right)^{\prime}=-\frac{r^{\lambda-2}}{\left(1+r^{2}\right)^{\lambda / 2}} \phi^{p}, p>1, \lambda>0$ : the $E$-solutions (regular at $r=0$ ), the $M$-solutions (singular at $r=0$ ) and the $F$-solutions (whose existence begins away from $r=0$ ). An essential tool is a transformation of the equation into a 2-dimensional asymptotically autonomous system, whose limit sets (by a theorem of H.R. Thieme) are the limit sets of Emden-Fowler systems, and serve as a characterization of the different solutions. The emphasis lies on the study of the $M$-solutions. The asymptotic expansions obtained make it possible to apply the results to the important question of stellar dynamics which solutions lead to galactic models (stationary solutions of the Vlasov-Poisson system) of finite radius and/or finite mass for different $p, \lambda$.
\end{abstract}

\section{Contents}

1. Introduction . . . . . . . . . . . . . . . 2

2. Preliminaries ........................ . . 6

2.1. The different types of solutions of the Matukuma equation . . . . . 6

2.2. Transformation to Lotka-Volterra systems . . . . . . . . . . . . . 8

2.3. Asymptotically autonomous systems . . . . . . . . . . . . . . . . . 9

2.4. The linearization of $\left(\mathrm{EFS}_{p, q}\right)$ at the stationary points $\ldots \ldots 10$

2.5. The linearization of $\left(\operatorname{EFS}_{p, 1}\right)$ at the stationary points . . . . . 11

2.6. The flow of $\left(\mathrm{MS}_{p, \lambda}\right) \ldots \ldots \ldots \ldots 11$

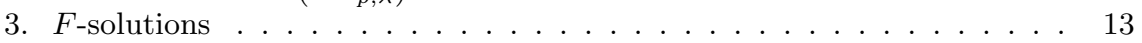

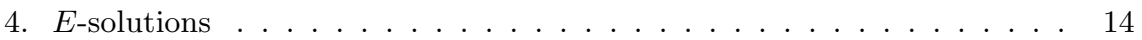

$5 . M$-solutions . . . . . . . . . . . . . . . . . . . . . . . . 19

5.1. The case $q>p \ldots \ldots \ldots \ldots \ldots$

5.1.1 The case $q>p+1 \ldots \ldots \ldots \ldots 21$

5.1 .2 The case $q=p+1 \ldots \ldots \ldots \ldots 24$ 
5.1.3 The case $p<q<p+1 \ldots \ldots \ldots \ldots \ldots$

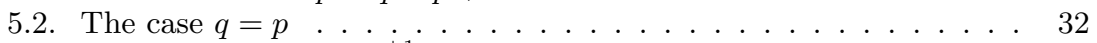

5.3. The case $q<p, q \neq \frac{p+1}{2} \ldots \ldots \ldots \ldots . \ldots \ldots$

5.4. The case $q=\frac{p+1}{2} \ldots \ldots \ldots \ldots$. . . . . . . . . . . . . . . . . . . . . . . . .

6. Solutions with $R<\infty \ldots \ldots \ldots$. . . . . . . . . . . . . . . 43

7. $P_{2}$-solutions (solutions of fast decay) . . . . . . . . . . . . . . . . 45

8. $P_{3}^{+}$-solutions (solutions of slow decay) . . . . . . . . . . 51

9. Global behaviour of the solutions and their radius and mass . . . . . . . 52

9.1. The case $q \leq \frac{p+1}{2} \ldots \ldots \ldots \ldots \ldots \ldots \ldots$

9.2. $M$-solutions for $q>\frac{p+1}{2} \ldots \ldots \ldots \ldots \ldots 4$

9.3. F-solutions . . . . . . . . . . . . . . . . . . 56

9.4. Known result on $E$-solutions . . . . . . . . . . . . . . . . 59

Appendix

\section{Introduction}

In the present paper we study the positive solutions $\phi=\phi(r)$ of the differential equation

$$
\frac{1}{r^{2}}\left(r^{2} \phi^{\prime}\right)^{\prime}=-\frac{r^{\lambda-2}}{\left(1+r^{2}\right)^{\lambda / 2}} \phi^{p}, \quad p>1, \lambda>0, \quad\left(\mathrm{M}_{p, \lambda}\right)
$$

on their maximal intervals $\left(R_{-}, R\right)$ of existence on the positive real line $\mathbb{R}^{+}$. For $\lambda=2$, these solutions are the radial solutions of the semilinear elliptic equation

$$
\Delta \phi=-\frac{1}{1+x^{2}} \phi^{p}
$$

on $\mathbb{R}^{3}$, which T. Matukuma proposed in 1930 for the description of certain stellar globular clusters in a steady state [22]. In this paper, we attach the name "Matukuma equation" to the more general form $\left(\mathrm{M}_{p, \lambda}\right)$. Earlier, with the appearance of R. Emden's book "Gaskugeln" in 1907, certain stellar dynamic models called polytropes had been introduced; their study has lead to the Emden-Fowler equation

$$
\frac{1}{r^{2}}\left(r^{2} \phi^{\prime}\right)^{\prime}=-r^{q-3} \phi^{p}, \quad p>1, q>1 . \quad\left(\mathrm{EF}_{p}, q\right)
$$

As was shown in [2], all these models are particular (classical) examples of a more general theory of time-independent spherically symmetric solutions of the Vlasov-Poisson system

$$
\left.\begin{array}{c}
\partial_{t} f+v \partial_{x} f-\partial_{x} U(t, x) \partial_{v} f=0 \\
\Delta U(t, x)=4 \pi \rho(t, x) \\
\rho(t, x):=\int f(t, x, v) d v
\end{array}\right\}
$$

$x, v \in \mathbb{R}^{3}$. Here $f=f(t, x, v) \geq 0$ is the distribution function of the considered system of gravitating mass in the space-velocity space $\mathbb{R}^{3} \times \mathbb{R}^{3}$, where 
$t \geq 0$ is the time, $U=U(t, x)$ the Newtonian potential and $\rho=\rho(t, x)$ the local density. Jeans' theorem says that the time-independent solutions $f$ in the case of spherical symmetry have the form $f=\Psi(E, F)$, where

$$
\begin{aligned}
& E:=\frac{v^{2}}{2}+U(r)=\frac{w^{2}}{2}+\frac{1}{2} \frac{F}{r^{2}}+U(r), \\
& F:=x^{2} v^{2}-(x v)^{2}
\end{aligned}
$$

are the local energy and the angular momentum in the coordinates

$$
r:=|x|, \quad w:=\frac{x \cdot v}{|x|}, \quad F:=x^{2} v^{2}-(x v)^{2} .
$$

Given $\Psi \geq 0$, a solution $U$ of Poisson's equation

$$
\frac{1}{r^{2}}\left(r^{2} U^{\prime}(r)\right)^{\prime}=4 \pi \rho(r),
$$

where

$$
4 \pi \rho(r)=\frac{8 \pi^{2}}{r^{2}} \iint_{\substack{w>0 \\ F>0}} \Psi\left(\frac{w^{2}}{2}+\frac{1}{2} \frac{F}{r^{2}}+U(r), F\right) d(w, F)=: h_{\Psi}(r, U(r)),
$$

together with $f:=\Psi\left(\frac{w^{2}}{2}+\frac{1}{2} \frac{F}{r^{2}}+U(r), F\right)$ and $\rho$ given by (1.2) leads to a solution of the (VPS) and hence to a spherically symmetric stationary stellar dynamic model $(U, f, \rho)$.

If we make the choice

$$
\Psi(E, F):=\left(E_{0}-E\right)_{+}^{n-3 / 2} F^{k}, \quad(\quad)_{+}=\text {positive part },
$$

where $E_{0}>0, n>1 / 2, k>-1$, such that $n+k>1$, (1.1) becomes

$$
\frac{1}{r^{2}}\left(r^{2} U^{\prime}(r)\right)^{\prime}=c r^{2 k}\left(E_{0}-u\right)_{+}^{n+k},
$$

where $c:=2^{k+7 / 2} \pi^{2} B\left(\frac{1}{2}, k+1\right) B\left(n-\frac{1}{2}, k+\frac{3}{2}\right)$ ( $B$ is the Beta function). The positive solutions $U<E_{0}$ of (1.3) correspond to the positive solutions $\phi$ of $\left(\mathrm{EF}_{p}, q\right)$ with $p:=n+k, q:=2 k+3$ via the substitution $\phi(r)=$ $E_{0}-U\left(c^{-\frac{1}{2 k+2}} r\right)$.

Camm's choice [6]

$$
\Psi(E, F):=\left(E_{0}-E-a F\right)_{+}^{n-3 / 2} F^{k},
$$

where $a>0$, leads to (1.1) in the form

$$
\frac{1}{r^{2}}\left(r^{2} U^{\prime}(r)\right)^{\prime}=\frac{c r^{2 k}}{\left(1+2 a r^{2}\right)^{k+1}}\left(E_{0}-U(r)\right)_{+}^{n+k} .
$$

The positive solutions $U<E_{0}$ correspond to the positive solutions $\phi$ of $\left(\mathrm{M}_{p, \lambda}\right)$ with $\lambda:=2 k+2$ via the substitution 


$$
\phi(r)=\left(\frac{c}{(2 a)^{k}+1}\right)^{\frac{1}{n+k-1}}\left(E_{0}-U\left(\frac{r}{\sqrt{2 a}}\right)\right)
$$

(see [2]).

Two physically most relevant problems are associated with the equations (1.1), (1.2), namely, to find those $\Psi$ and those initial conditions for (1.1), for which

A) the local density $\rho(r)$ has bounded support,

B) the total mass $M:=4 \pi \int \rho(r) r^{2} d r$ is finite.

These problems had been completely solved for all positive solutions of the Emden-Fowler equation $\left(\mathrm{EF}_{p}, q\right)$ with $p, q>1$ in [4] and investigated in the relativistic case in [1]; the results have been applied in the stability theory for the (VPS) in [3] and [10]. The problem in full generality seems to be extremely difficult, as pointed out in [2]. Further partial answers have been given by G. Rein and A.D. Rendall $[27-29,31,32]$ for the (VPS) and the Vlasov-Einstein system, where this problem also occurs, and by M. Heinzle, A.D. Rendall and C. Uggla [11]. In [32] it is proven that the induced $f$ has compact support and finite mass if $\Psi$ is of the form

$$
\Psi(E, F)=\Psi_{1}(E) \cdot F^{l},
$$

where $\Psi_{1}(E)=c \cdot\left(E_{0}-E\right)_{+}^{k}+O\left(\left(E_{0}-E\right)_{+}^{k+\delta}\right)\left(E \rightarrow E_{0}\right)$, with $k>-1$, $l>-1 / 2, k+l+1 / 2>0, k<l+3 / 2$ and $c, \delta>0$ (also the case of the Vlasov-Einstein equations is considered). The result is generalized in [11] to nonnegative functions $\Psi_{1} \in C^{1}(0, \infty)$ such that $\Psi_{1}^{\prime}$ is locally bounded in $(0, \infty)$ and $\Psi_{1}^{\prime}(E) \leq \mathrm{const} \cdot E^{k^{\prime}}$ for some $k^{\prime}>-2$ near $E=0$. The papers $[4,11]$ reduce the question to a phase space analysis for solutions of systems of ordinary differential equations.

As for the Matukuma equation and related equations, almost the entire work has been done for the $E$-solutions, beginning with the study of Matukuma's own conjectures about these solutions [24, p. 2]; among those, whose work is more closely related to the present paper, we mention $\mathrm{W}$. Kawano, M. Kwong, Y. Li, W.-M. Ni, Y. Santanilla, E. Yanagida, S. Yotsutani [12-20,23,24,35-38]. In Section 9.4, we refer to their work in more detail.

One aim of this paper is to develop a comprehensive theory of all positive solutions $\phi$ of the Matukuma equation. The three different types of solutions known from the Emden-Fowler equation also exist for the Matukuma equation, namely the $E$-solutions (which are regular at $r=0$ ), the $M$-solutions (singular at $r=0$ ) and the $F$-solutions (with $R_{-}>0$ ). Particularly the $M$-solutions have an extremely rich structure. They correspond to stellar dynamic models with a singular, but integrable local density at $r=0$. In this context we point out that the initial value problem for the (VPS) has 
been satisfactorily solved for bounded (smooth) initial data, but for singular data a proof for global existence is still open: the singular stationary solutions, which we now know, are not contained in the class of initial conditions of any theorem yielding global existence of classical solutions [30]. To fill this gap is certainly a promising new line of research for the (VPS). Let us also mention the following result pertaining to the Vlasov-Einstein system [33]: If a spherically symmetric solution develops a singularity at all then the first singularity must appear at the center of the symmetry.

For the purpose of the latter applications, we derive asymptotic expansions at $r=0$ and at $r=R=\infty$, even more accurate ones than those known for the Emden-Fowler equation [4,5]. A first and powerful tool is the method which was invented for a precise asymptotic study of (not necessarily radial) solutions of certain semilinear equations at infinity $[14,16]$. This method begins with rough estimates and improves the accuracy step by step to the desired extent. We show the applicability of this method at $r=0$ for the $E$ - and $M$-solutions. In the latter case, we found in Section 5.1 that for $q>p$ every $M$-solution $\phi$ admits a splitting of the form $\phi=S+\Theta$, where $S$ is a singular and $\Theta$ is a regular part, which is an " $E$-solution" of a certain (singular) second order equation. For $p<q<p+1, S$ has the form of a "Laurent expansion" with respect to $r^{q-p}$ with finitely many singular terms.

The aforementioned paper [4] was based on the substitution

$$
u(t)=r^{q-2} \frac{\phi^{p}(r)}{-\phi^{\prime}(r)}, \quad v(t)=r \frac{-\phi^{\prime}(r)}{\phi(r)}, \quad r=e^{t},
$$

which maps the solutions of $\left(\mathrm{EF}_{p}, q\right)$ onto the solutions $\varphi=(u, v)$ of the Lotka-Volterra system

$$
\begin{aligned}
& \dot{u}=u(q-u-p v), \\
& \dot{v}=v(-1+u+v) .
\end{aligned}
$$

The $F$-, $E$ - and $M$-solutions and those with $R<\infty$ could be characterized and analyzed by their limit sets $L^{+}(\varphi), L^{-}(\varphi)$ in the quadrant $\mathbb{R}^{+} \times \mathbb{R}^{+}$, which can be found by means of the Poincaré-Bendixson theorem. If one applies this substitution to solutions of the $\left(\mathrm{M}_{p, \lambda}\right)$, one gets

$$
\begin{aligned}
& \dot{u}=u(q(t)-u-p v), \\
& \dot{v}=v(-1+u+v) .
\end{aligned}
$$

The coefficient $q(t)$ is time-dependent, but the limits $\lim _{t \rightarrow-\infty} q(t)=1+$ $\lambda, \lim _{t \rightarrow+\infty} q(t)=1$ exist, and this fact makes this system an asymptotically autonomous one. We can make use of a theorem of H.R. Thieme [34], who has proved a structure theorem for the limit sets of asymptotically autonomous systems and we profit from the fact that the limiting systems in our case are just particular systems of Emden-Fowler type $\left(\mathrm{EFS}_{p}, q\right)$, which had been completely studied in [4]. 
We have given an outlook of what the reader can expect in Sections 3, 4 and 5 which are devoted to the $F$-, $E$ - and $M$-solutions, after the necessary preparations in Section 2. Sections 6,7 and 8 are devoted to the solutions with $R<\infty$, and with rapid and slow decay at $R=\infty$. To derive the asymptotic expansions at $R=\infty$, we present another method, which makes use of the visualization of the solution by the phase space analysis, the representation of the $\left(\mathrm{MS}_{p}, \lambda\right)$ as a time-dependent perturbation of the $\left(\mathrm{EF}_{p}, q\right)$ and elements of the proof the Hartman-Grobman theorem. Section 9 addresses the questions $\mathrm{A}$ ) and $\mathrm{B}$ ) of finite $R$ (i.e., the existence of a zero of $\phi$ ) and finite $M$. The formula

$$
M=\bar{c} \lim _{r \downarrow R_{-}} r^{2} \phi^{\prime}(r)-\bar{c} \lim _{r \uparrow R} r^{2} \phi^{\prime}(r),
$$

where $\bar{c}:=\left[\frac{(2 a) \frac{q-p}{2}}{c}\right]^{\frac{1}{p-1}}$ shows that B) is a question of the asymptotic expansions at $r=0$ and $R=\infty$. Though there are solutions whose graph intersects itself, we have numerous sets of initial conditions for which the $F$-, $E$ - and $M$-solutions have finite or infinite $R$ and $M$ (see also the appendix with graphs which illustrate our results). Finally, we remark that it follows from Section 2.2 that the $\left(\mathrm{EFS}_{p}, q\right)$ plays the key role of being the limit system for many other equations. But we refrain from extending our results to more general settings.

\section{Preliminaries}

\subsection{The different types of solutions of the Matukuma equation}

Let $K$ be a positive function in $C^{1}\left(\mathbb{R}^{+}\right)$with $r^{2} K(r)$ bounded away from zero for $r \rightarrow \infty$, and $p>1$. Let $\phi:\left(R_{-}, R\right) \rightarrow(0, \infty)$ be a maximal solution of the more general equation

$$
\frac{1}{r^{2}}\left(r^{2} \phi^{\prime}\right)^{\prime}=-K(r) \phi^{p} \quad \text { on } \mathbb{R}^{+}:=(0, \infty) .
$$

Then $0 \leq R_{-}<R \leq \infty$. Let $r_{0} \in\left(R_{-}, R\right)$ and

$$
H(r):=\phi^{\prime}\left(r_{0}\right) r_{0}^{2}-\int_{r_{0}}^{r} s^{2} K(s) \phi(s)^{p} d s \quad \text { on }\left(R_{-}, R\right) .
$$

Then $\phi^{\prime}(r)=\frac{H(r)}{r^{2}}$ and $H^{\prime}(r)=-r^{2} K(r) \phi(r)^{p}<0$ on $\left(R_{-}, R\right)$. Hence the limit

$$
H_{0}:=\lim _{r \downarrow R_{-}} H(r) \in(-\infty,+\infty]
$$

exists. If $H_{0}>0$, then $R_{-}>0$. In fact, if $R_{-}=0$, then there exists $r_{1} \in\left(0, r_{0}\right)$ with $H\left(r_{1}\right)>0$ and $H(r)>H\left(r_{1}\right)$ on $\left(0, r_{1}\right]$, hence

$$
\begin{aligned}
\phi(r) & =\phi\left(r_{0}\right)-\int_{r}^{r_{0}} \phi^{\prime}(s) d s=\phi\left(r_{0}\right)-\int_{r}^{r_{1}} \frac{H(s)}{s^{2}} d s-\int_{r_{1}}^{r_{0}} \phi^{\prime}(s) d s \\
& \leq \phi\left(r_{0}\right)-H\left(r_{1}\right) \int_{r}^{r_{1}} \frac{d s}{s^{2}}-\int_{r_{1}}^{r_{0}} \phi^{\prime}(s) d s \longrightarrow-\infty \quad(r \rightarrow 0),
\end{aligned}
$$


which is a contradiction. Hence $R_{-}>0$. In this case there exists an $R_{0} \in$ $\left(R_{-}, R\right)$ with $H\left(R_{0}\right)=0=\phi^{\prime}\left(R_{0}\right)$ : Otherwise we would have $H>0$ on $\left(R_{-}, R\right), H$ and $\phi^{\prime}$ are bounded and $\phi^{\prime}>0$. This implies $R=\infty$ and $\phi$ is increasing, so that $H(r) \rightarrow-\infty$ by the assumption on $K$. This is a contradiction, and $\phi^{\prime}<0$ on $\left(R_{0}, R\right)$ and $H>0$ and $\phi^{\prime}>0$ on $\left(R_{-}, R_{0}\right)$ by letting $r_{0}=R_{0}$ in (2.1.2). Then $\lim _{r \rightarrow R_{-}} \phi^{\prime}(r)$ is finite and $\lim _{r \rightarrow R_{-}} \phi(r)=$ 0 . If $H_{0} \leq 0$, then $R_{-}=0$. Assume $R_{-}>0$. Then since $H^{\prime}<0$ on $\left(R_{-}, R_{0}\right)$ there exists $r_{2} \in\left(R_{-}, r_{1}\right)$ so that $H\left(r_{2}\right)<0, \phi^{\prime}\left(r_{2}\right)<0, H$ and $\phi^{\prime}$ are bounded on $\left(R_{-}, r_{2}\right)$ and $\phi$ could be extended beyond $R_{-}$. Hence $R_{-}=0$, $\phi^{\prime}<0$ on $(0, R)$ and the limit

$$
\lim _{r \downarrow 0} \phi(r) \in(0, \infty]
$$

exists. In this case, we define $R_{0}:=0$ and have

$$
R_{0}=\inf \left\{r \in\left(R_{-}, R\right): \phi^{\prime}(r)<0\right\}
$$

for all solutions.

Now we classify the solutions as follows. We have shown: $H_{0}>0 \Longleftrightarrow$ $R_{-}>0 \Longleftrightarrow R_{0}>R_{-}$. In this case we call $\phi$ an $F$-solution. Furthermore: $H_{0} \leq 0 \Longleftrightarrow R_{-}=0 \Longleftrightarrow R_{0}=0$. In this case, if $\lim _{r \downarrow 0} \phi(r)<\infty$, we call $\phi$ an $E$-solution, if $\lim _{r \downarrow 0} \phi(r)=\infty$, an $M$-solution.

Particular examples of (2.1.1) are the Emden-Fowler equation

$$
\frac{1}{r^{2}}\left(r^{2} \phi^{\prime}\right)^{\prime}=-r^{q-3} \phi^{p}, \quad p>1, q>1, \quad\left(\mathrm{EF}_{p}, q\right)
$$

and the Matukuma equation

$$
\frac{1}{r^{2}}\left(r^{2} \phi^{\prime}\right)^{\prime}=-\frac{r^{\lambda-2}}{\left(1+r^{2}\right)^{\lambda / 2}} \phi^{p}, \quad p>1, \lambda>0 . \quad\left(\mathrm{M}_{p}, \lambda\right)
$$

We will show that the three types of solutions also exist for this equation. As for the $E$-solutions, we will frequently make use of the following lemma which is part of the more general Theorem 5.6 in [2, p. 172], see also [24].

Lemma 2.1. Let $\alpha \in \mathbb{R}$ and let $h:(0, \infty) \times \mathbb{R} \rightarrow \mathbb{R}$ satisfy the following conditions:

(i) $h \in C^{1}((0, \infty) \times \mathbb{R})$,

(ii) $\operatorname{sh}(\cdot, \alpha) \in L_{\text {loc }}^{1}[0, \infty)$,

(iii) there exists a number $\delta>0$ and a function

$$
L_{0 \alpha}:(0, \delta) \rightarrow[0, \infty] \text { with } r L_{0 \alpha} \in L^{1}[0, \delta],
$$

such that for all $r \in(0, \delta)$ and $u_{1}, u_{2} \in[\alpha-\delta, \alpha+\delta]$ we have

$$
\left|h\left(r, u_{1}\right)-h\left(r, u_{2}\right)\right| \leq L_{0 \alpha}(r)\left|u_{1}-u_{2}\right| .
$$

Then the initial value problem

$$
\frac{1}{r^{2}}\left(r^{2} \phi^{\prime}\right)^{\prime}=h(r, \phi), \phi(0)=\alpha
$$


has a unique (maximal) solution $\phi$ on $(0, R)$ with $\phi(0):=\lim _{r \rightarrow 0} \phi(r)=$ $\alpha$.

If, for some $\gamma \geq 0$, we have

$$
\frac{1}{r^{\gamma+1}} \int_{0}^{r} s^{2} L_{0 \alpha}(s) d s=O(1)(r \rightarrow 0),
$$

then

$$
r^{1-\gamma} \phi^{\prime}(r)-\frac{1}{r^{\gamma+1}} \int_{0}^{r} s^{2} h(s, \alpha) d s \rightarrow 0(r \rightarrow 0) .
$$

\subsection{Transformation to Lotka-Volterra systems}

In this section we consider solutions $\phi$ of (2.1.1) on their intervals $J_{\phi}:=$ $\left(R_{0}, R\right)$. We define

$$
u(t):=r K(r) \frac{\phi^{p}(r)}{-\phi^{\prime}(r)}, \quad v(t):=r \frac{-\phi^{\prime}(r)}{\phi(r)}, \quad r:=e^{t} .
$$

Then $\varphi:=(u, v): I_{\varphi} \rightarrow \mathbb{R}^{+} \times \mathbb{R}^{+}, I_{\varphi}:=\ln J_{\phi}$ is a maximal solution of the system

$$
\begin{aligned}
& \dot{u}=u(q(t)-u-p v), \\
& \dot{v}=v(-1+u+v), \text { with } q(t):=3+\frac{r K^{\prime}(r)}{K(r)} .
\end{aligned}
$$

$\mathbb{R}^{+} \times \mathbb{R}^{+}$is an invariant set of this system (the positive $u$ - and $v$-axes are invariant). The inverse is

$$
\phi(r)=\left(\frac{u(\ln r) v(\ln r)}{r^{2} K(r)}\right)^{\frac{1}{p-1}} .
$$

For $\left(\mathrm{EF}_{p}, q\right)$ we have $K(r)=r^{q-3}$ and thus obtain

$$
\begin{aligned}
& \dot{u}=u(q-u-p v), \\
& \dot{v}=v(-1+u+v) .
\end{aligned}
$$

For $\left(\mathrm{M}_{p}, \lambda\right)$ we have

$$
K(r)=\frac{r^{\lambda-2}}{\left(1+r^{2}\right)^{\lambda / 2}}
$$

and

$$
u(t)=\frac{r^{\lambda-1}}{\left(1+r^{2}\right)^{\lambda / 2}} \frac{\phi^{p}(r)}{-\phi^{\prime}(r)}, \quad v(t)=r \frac{-\phi^{\prime}(r)}{\phi(r)}
$$

satisfy

$$
\begin{aligned}
& \dot{u}=u(q(t)-u-p v), \\
& \dot{v}=v(-1+u+v),
\end{aligned}
$$

with

$$
q(t)=3+\frac{\lambda-2-2 r^{2}}{1+r^{2}}=1+\frac{\lambda}{1+r^{2}} .
$$


The inverse is

$$
\begin{aligned}
& \phi(r)=\left(\left(1+r^{2}\right)^{\lambda / 2} r^{-\lambda} u(\ln r) v(\ln r)\right)^{\frac{1}{p-1}} \\
& \phi^{\prime}(r)=-\frac{1}{r} \phi(r) \cdot v(\ln r)
\end{aligned}
$$

and the limits

$$
\lim _{t \rightarrow-\infty} q(t)=1+\lambda, \quad \lim _{t \rightarrow+\infty} q(t)=1
$$

exist. We define $q:=1+\lambda$. Then $\left(\mathrm{MS}_{p, \lambda}\right)$ is asymptotically autonomous with respect to $\left(\mathrm{EFS}_{p, q}\right)$ for $t \rightarrow-\infty$ and to $\left(\mathrm{EFS}_{p, 1}\right)$ for $t \rightarrow+\infty$ in the sense of the next section. The following decompositions:

$$
\begin{aligned}
q(t) & =q-(q-1) \frac{e^{2 t}}{1+e^{2 t}}=q-(q-1) e^{2 t}+(q-1) \frac{e^{4 t}}{1+e^{2 t}} \\
& =1+(q-1) \frac{e^{-2 t}}{1+e^{-2 t}}=1+(q-1) e^{-2 t}-(q-1) \frac{e^{-4 t}}{1+e^{-2 t}}
\end{aligned}
$$

are useful for $t \rightarrow-\infty$ and $t \rightarrow+\infty$ respectively.

In the sequel unless otherwise stated, $\phi$ denotes a solution of $\left(\mathrm{M}_{p, \lambda}\right)$ on $\left(R_{0}, R\right)$ with $0 \leq R_{0}<R \leq \infty$ and $\varphi=(u, v)$ is the associated solution of $\left(\mathrm{MS}_{p, \lambda}\right)$ on $\left(T_{0}, T\right)$ with $-\infty \leq T_{0}=\ln R_{0}<T=\ln R \leq \infty$. And it is always assumed that $p, q>1, \lambda=q-1>0$.

\subsection{Asymptotically autonomous systems}

A system of ordinary differential equations in the plane $\mathbb{R}^{2}$

$$
\dot{x}=f(t, x)
$$

is called asymptotically autonomous with respect to the autonomous system

$$
\dot{x}=g(x)
$$

for $t \rightarrow+\infty$, if $f(t, x) \longrightarrow g(x)$ as $t \rightarrow+\infty$ uniformly on compact subsets of $\mathbb{R}^{2}$. As usual we use $L^{+}(\varphi), L^{-}(\varphi)$ for the positive, negative limit set and $C^{+}(\varphi), C^{-}(\varphi)$ for the positive, negative half trajectory of an orbit $\varphi$.

For asymptotically autonomous systems H.R. Thieme [34] has proved the following theorem.

Theorem 2.2. (H.R. Thieme) Let $\varphi$ on $(\tau, \infty)$ be a solution of (NA) and assume $L^{+}(\varphi) \subset K$ for a compact subset $K$ of $\mathbb{R}^{2}$. If $K$ only contains finitely many stationary points of $(\mathrm{A})$, then exactly one of the following (distinct) 3 cases occurs:

(A) $L^{+}(\varphi)$ consists of a stationary point of $(\mathrm{A})$,

(B) $L^{+}(\varphi)$ is the union of periodic orbits of (A) and possibly center points of (A), which are encircled by periodic orbits of (A) lying in $L^{+}(\varphi)$, 
(C) $L^{+}(\varphi)$ contains stationary points of (A) which are cyclically chained by orbits of (A) lying in $L^{+}(\varphi)$.

A similar statement holds for $t \rightarrow-\infty$.

As is clear from the last paragraph, we can apply Thieme's theorem to an analysis of $\left(\mathrm{MS}_{p, \lambda}\right)$ with its autonomous limit systems $\left(\operatorname{EFS}_{p, q}\right)$ and $\left(\mathrm{EFS}_{p, 1}\right)$ being in the good position that the limit sets of $\left(\operatorname{EFS}_{p, q}\right)$ are completely known from [4].

\subsection{The linearization of $\left(\operatorname{EFS}_{p, q}\right)$ at the stationary points}

The system $\left(\operatorname{EFS}_{p, q}\right), p, q>1$, has the following stationary points: $P_{1}=(0,0), P_{2}=(0,1), P_{3}^{-}=(q, 0) ;$ for $q<p: P_{4}=\left(\frac{p-q}{p-1}, \frac{q-1}{p-1}\right)$. If the stationary point is $P=(a, b)$, and if we introduce the coordinates $\bar{u}:=u-a, \bar{v}:=v-b$, then we can write $\left(\mathrm{MS}_{p, \lambda}\right)$ as a time-dependent perturbation of $\left(\operatorname{EFS}_{p, q}\right)$ :

$$
\left(\begin{array}{c}
\bar{u} \\
\bar{v}
\end{array}\right)=A\left(\begin{array}{c}
\bar{u} \\
\bar{v}
\end{array}\right)+\left(\begin{array}{c}
-\bar{u}^{2}-p \bar{u} \bar{v} \\
\bar{u} \bar{v}+\bar{v}^{2}
\end{array}\right)+\left(\begin{array}{c}
-(q-1)(\bar{u}+a) \frac{e^{2 t}}{1+e^{2 t}} \\
0
\end{array}\right),
$$

with

$$
A:=\left(\begin{array}{cc}
q-2 a-p b & -p a \\
b & a+2 b-1
\end{array}\right) .
$$

For the following, we consider the linear part of (2.4.1).

For $P_{1}$ we have $A=\left(\begin{array}{cc}q & 0 \\ 0 & -1\end{array}\right)$ with eigenvalues $\lambda_{1}=q, \lambda_{2}=-1$ and eigenvectors $\xi_{1}=(0,1), \xi_{2}=(1,0)$, and $P_{1}$ is a saddle.

For $P_{2}$ we have $A=\left(\begin{array}{cr}q-p & 0 \\ 1 & 1\end{array}\right)$ with $\lambda_{1}=q-p, \lambda_{2}=1$ and $\xi_{1}=$ $(q-p-1,1), \xi_{2}=(0,1)$. That is, for $q>p P_{2}$ is an unstable improper node. In particular, for $q>p+1: \quad 0<\lambda_{2}<\lambda_{1}$ and $P_{2}$ is a 2-tangential improper node with main direction $\xi_{2}$; for $q=p+1$ : $\quad 0<\lambda_{2}=\lambda_{1}=1$ and $P_{2}$ is a 1-tangential node with direction $(0,1)$; for $p<q<p+1$ : $\quad 0<\lambda_{1}<\lambda_{2}$ and $P_{2}$ is a 2 -tangential node with main direction $-\xi_{1}$; for $q=p: \lambda_{1}=0$ and $P_{2}$ is an unstable 2-tangential node with main direction $(1,-1)$; for $q<p: P_{2}$ is a saddle.

For $P_{3}^{-}$we have $A=\left(\begin{array}{cc}-q & -p q \\ 0 & q-1\end{array}\right)$ with $\lambda_{1}=-q, \lambda_{2}=q-1$ and $\xi_{1}=(1,0), \xi_{2}=\left(-1,\left(2-\frac{1}{q}\right) \frac{1}{p}\right)$, and $P_{3}^{-}$is a saddle.

For

$$
\begin{aligned}
& q<p: P_{4}=\left(\frac{p-q}{p-1}, \frac{q-1}{p-1}\right)=:\left(u_{4}, v_{4}\right)\left(u_{4}+v_{4}=1\right), \\
& A=\left(\begin{array}{cc}
v_{4}-1 & p\left(v_{4}-1\right) \\
v_{4} & v_{4}
\end{array}\right), \lambda_{1,2}=v_{4}-\frac{1}{2} \pm \frac{1}{2} \sqrt{\Theta\left(v_{4}\right)}
\end{aligned}
$$


where $\Theta(s)=1-4 p s(1-s)$ with two distinct roots $\eta_{1,2}=\frac{1}{2}\left(1 \pm \sqrt{1-\frac{1}{p}}\right)$. For $v_{4}>\frac{1}{2} \Leftrightarrow q>\frac{p+1}{2}$ we have $\operatorname{Re} \lambda_{i}>0, i=1,2$ and $P_{4}$ is unstable. In particular, for $\eta_{1} \leq v_{4}<1, P_{4}$ is an improper node and for $\frac{1}{2}<v_{4}<\eta_{1}$ a spiral point. For $v_{4}=\frac{1}{2} \Leftrightarrow q=\frac{p+1}{2}$ we have $\lambda_{1,2}= \pm \frac{1}{2} \sqrt{p-1} i$ and $P_{4}$ is a center. For $0<v_{4}<\frac{1}{2} \Leftrightarrow q<\frac{p+1}{2}$ we have $\operatorname{Re} \lambda_{i}<0, i=1,2$ and $P_{4}$ is stable. In particular, for $\eta_{2}<v_{4}<\frac{1}{2} P_{4}$ is a spiral point and for $0<v_{4} \leq \eta_{2}$ $P_{4}$ is an improper node.

\subsection{The linearization of $\left(\mathrm{EFS}_{p, 1}\right)$ at the stationary points}

The system $\left(\mathrm{EFS}_{p, 1}\right), p>1$, has the following stationary points: $P_{1}=$ $(0,0), P_{2}=(0,1), P_{3}^{+}=(1,0)$. With the notation of Section 2.4 (here we have $q=1$ )

$\left(\begin{array}{c}\bar{u} \\ \bar{v}\end{array}\right)=A\left(\begin{array}{c}\bar{u} \\ \bar{v}\end{array}\right)+\left(\begin{array}{cc}-\bar{u}^{2}-p \bar{u} \bar{v} \\ \bar{u} \bar{v}+\bar{v}^{2}\end{array}\right)$, where $A:=\left(\begin{array}{cc}-1-2 a-p b & -p a \\ b & -1+a+2 b\end{array}\right)$.

For $P_{1}$ we have $A=\left(\begin{array}{cc}1 & 0 \\ 0 & -1\end{array}\right)$ and $P_{1}$ is a saddle.

For $P_{2}$ we have $A=\left(\begin{array}{cc}1-p & 0 \\ 1 & 1\end{array}\right)$ with eigenvalues $\lambda_{1}=1-p<0, \lambda_{2}=1$ and eigenvectors $\xi_{1}=(p,-1), \xi_{2}=(0,1)$ and $P_{2}$ is a saddle.

For $P_{3}^{+}$we have $A=\left(\begin{array}{cc}-1 & -p \\ 0 & 0\end{array}\right)$ with $\lambda_{1}=-1, \lambda_{2}=0$ and $\xi_{1}=(1,0)$, $\xi_{2}=(-p, 1)$ and and $P_{3}^{+}$is a stable 2 -tangential improper node with main direction $\xi_{2}$.

\subsection{The flow of $\left(\mathrm{MS}_{p, \lambda}\right)$}

From (2.2.6), (2.2.7) we have $\dot{q}(t)<0$, so that $q(\cdot)$ strictly decreases from $q(-\infty)=q$ to $q(+\infty)=1$ on $\overline{\mathbb{R}}:=\{-\infty \leq t \leq+\infty\}$. We define

$$
\begin{aligned}
S(t, u, v) & :=q(t)-u-p v, \quad t \in \overline{\mathbb{R}}, \\
W(u, v) & :=-1+u+v,
\end{aligned}
$$

and have $\left(\mathrm{MS}_{p, \lambda}\right)$ in the form

$$
\begin{aligned}
& \dot{u}=u S(t, u, v), \\
& \dot{v}=v W(u, v) .
\end{aligned}
$$

For functions $F: \mathbb{R}^{+} \times \mathbb{R}^{+} \rightarrow \mathbb{R}$, we let

$$
F_{0}:=F^{-1}\{0\}, \quad F_{+}:=F^{-1}\left\{\mathbb{R}^{+}\right\}, \quad F_{-}:=F^{-1}\left\{\mathbb{R}^{-}\right\} .
$$

$S_{0}(t), t \in \overline{\mathbb{R}}$, is the straight line $v=-\frac{1}{p} u+\frac{q(t)}{p}$ with intercepts $(q(t), 0)$ and $\left(0, \frac{q(t)}{p}\right)$. It moves downward in the closed strip $Z$ between $S_{0}(-\infty)$ 
and $S_{0}(+\infty)$ when $t$ runs from $-\infty$ to $+\infty$. $W_{0}$ is the fixed straight line $v=1-u$. Furthermore, we define

$$
G(u, v):=u+\frac{p+1}{2}(v-1) .
$$

$G_{0}$ is the straight line $v=-\frac{2}{p+1} u+1$ with intercepts $\left(\frac{p+1}{2}, 0\right)$ and $(0,1)$. If $q>p, P_{2} \in Z$. If $q>\frac{p+1}{2}$, we define $T^{*} \in \mathbb{R}$ by $q\left(T^{*}\right):=\frac{p+1}{2}$ and $T^{*}=:-\infty$ for $q \leq \frac{p+1}{2}$. Then $S_{0}(t) \subset G_{-}$for $t \geq T^{*}$.

Lemma 2.3. Let $\varphi$ be a solution of $\left(\mathrm{MS}_{p, \lambda}\right)$ on $\left(T_{0}, T\right)$. Let $q>\frac{p+1}{2}$.

$$
\begin{aligned}
& \text { If } \varphi\left(t_{0}\right) \in G_{+} \text {for } t_{0}<T^{*}, \quad \text { then } \varphi(t) \in G_{+} \text {for } t_{0} \leq t \leq T^{*} . \\
& \text { If } \varphi\left(t_{0}\right) \in G_{-} \cup G_{0} \text { for } t_{0} \leq T^{*}, \quad \text { then } \varphi(t) \in G_{-} \text {for } t<t_{0} . \\
& \text { If } \varphi\left(t_{0}\right) \in G_{-} \cup G_{0} \text { for } t_{0} \geq T^{*}, \quad \text { then } \varphi(t) \in G_{-} \text {for } t>t_{0} . \\
& \text { If } \varphi\left(t_{0}\right) \in G_{+} \cup G_{0} \text { for } t_{0}>T^{*}, \quad \text { then } \varphi(t) \in G_{+} \text {for } T^{*} \leq t<t_{0} . \\
& \text { For } q \leq \frac{p+1}{2} \text { the last two statements are true for all } t_{0} .
\end{aligned}
$$

Proof. We have

$$
\begin{aligned}
\frac{d}{d t} G(\varphi(t)) & =\dot{u}+\frac{p+1}{2} \dot{v}=u(q(t)-u-p v)+\frac{p+1}{2} v(-1+u+v) \\
& =u\left(q(t)-u+\frac{-p+1}{2} v\right)+\frac{p+1}{2} v(-1+v) .
\end{aligned}
$$

If $\varphi(t) \in G_{0}$, then $-u=\frac{p+1}{2}(v-1)$, hence

$$
\begin{aligned}
\left.\frac{d}{d t} G(\varphi(t))\right|_{\varphi(t) \in G_{0}} & u\left(q(t)+\frac{p+1}{2}(v-1)+\frac{-(p+1)}{2} v-v\right) \\
= & u\left(q(t)-\frac{p+1}{2}\right) \\
& =\left\{\begin{array}{cr}
u\left(q(t)-q\left(T^{*}\right)\right)>0 \text { for } q>\frac{p+1}{2} \text { and } t<T^{*} \\
u\left(q(t)-q\left(T^{*}\right)\right)<0 \text { for } q>\frac{p+1}{2} \text { and } t>T^{*} \\
<0 & \text { for } q \leq \frac{p+1}{2} \text { and all } t .
\end{array}\right.
\end{aligned}
$$

This proves the assertions for $t_{0} \neq T^{*}$. If $q>\frac{p+1}{2}$, then from the above and an easy calculation we have

$$
\left.\begin{array}{l}
\left.\frac{d}{d t} G(\varphi(t))\right|_{\left\{\varphi\left(T^{*}\right) \in G_{0}\right\}}=0, \\
\left.\frac{d^{2}}{d t^{2}} G(\varphi(t))\right|_{\left\{\varphi\left(T^{*}\right) \in G_{0}\right\}}=\frac{\left(q-\frac{p+1}{2}\right)(p-1)(p+1)}{q-1}\left(v\left(T^{*}\right)-1\right)<0,
\end{array}\right\}
$$

which shows the claim also for $t_{0}=T^{*}>-\infty$.

Remark 1. Essentially, the foregoing proof consists of the calculation

$$
u S(t, u, v)+\left.\frac{p+1}{2} v W(u, v)\right|_{(u, v) \in G_{0}}=u\left(q(t)-\frac{p+1}{2}\right) .
$$


The left-hand side is equal to $\left.\langle n,(\dot{u}, \dot{v})\rangle\right|_{(u, v) \in G_{0}}$, where $n:=\left(1, \frac{p+1}{2}\right)$ is a vector normal to $G_{0}$. The geometric interpretation of the result is then clear: the flow is directed outward of $G_{-}$for $q>\frac{p+1}{2}, t<T^{*}$, and inward for $t>T^{*}$, and for $q \leq \frac{p+1}{2}$ for all $t$.

Lemma 2.4. No solution $\varphi$ of $\left(\mathrm{MS}_{p, \lambda}\right)$ can converge to $P_{1}$ for $t \rightarrow-\infty$ nor for $t \rightarrow+\infty$.

Proof. We have $P_{1} \subset S_{+}(t) \cap W_{-}$, where $\dot{u}(t)>0$ and $\dot{v}(t)<0$ for all $t$.

\section{F-solutions}

For $p, q>1$ we consider solutions $\phi$ of $\left(\mathrm{M}_{p, \lambda}\right)$ on $\left(R_{0}, R\right), 0 \leq R_{0}<R \leq$ $\infty$, and their corresponding solutions $\varphi$ of $\left(\mathrm{MS}_{p, \lambda}\right)$ on $\left(T_{0}, T\right)$. The following characterization of $F$-solutions implies their existence.

Theorem 3.1. The following conditions are equivalent:

a) $\phi$ is an F-solution, that is, $R_{0}>0$.

b) $T_{0}>-\infty$.

c) $C^{-}(\varphi)$ is unbounded as $t \downarrow T_{0}$.

d) There exists $t_{0} \in\left(T_{0}, T\right)$ such that $u\left(t_{0}\right) \geq q$.

e) $u(t) \uparrow \infty$ as $t \downarrow T_{0}$.

In this case, $v(t) \downarrow 0$ as $t \downarrow T_{0}$.

Proof. a) $\leftrightarrow$ b) follows from the definitions for $T_{0}$ and $R_{0}$.

b) $\rightarrow$ c): If $C^{-}(\varphi)$ were bounded, the solution $\varphi$ could be extended to a solution $\bar{\varphi}:\left[T_{0}, T\right) \rightarrow \mathbb{R}_{0}^{+} \times \mathbb{R}_{0}^{+}$. But $\bar{\varphi}\left(T_{0}\right)$ is not a point on the axes, hence $\bar{\varphi}\left(T_{0}\right) \in \mathbb{R}^{+} \times \mathbb{R}^{+}$and $\varphi$ could again be extended beyond $T_{0}$. This is a contradiction.

c) $\rightarrow \mathrm{d}$ ): If d) were not true, $u$ would be bounded and $v$ must be unbounded as $t \downarrow T_{0}$. Hence there exists $t_{0} \in\left(T_{0}, T\right)$ with $v_{0}:=v\left(t_{0}\right)>1$. We have $\dot{v}>v(-1+v)$. The solution of the initial-value problem $\dot{z}=$ $z(-1+z), z\left(t_{0}\right)=v_{0}$ is

$$
z(t)=\frac{1}{1-c e^{t}} \quad \text { with } \quad c:=\frac{v_{0}-1}{v_{0}} e^{-t_{0}} \quad \text { on }\left(-\infty, \ln \frac{1}{c}\right)
$$

and we have $v(t) \leq z(t), T_{0}<t \leq t_{0}$. Because $z(t) \rightarrow 1(t \rightarrow-\infty) v$ is bounded on $\left(T_{0}, t_{0}\right]$. This is a contradiction.

$\mathrm{d}) \rightarrow \mathrm{b})$, e): Let $u_{0}:=u\left(t_{0}\right)$. We have $\dot{u}<u(q(t)-u)<u(q-u)$. The solution of the initial-value problem $\dot{z}=z(q-z), z\left(t_{0}\right)=u_{0}$ is

$$
z(t)=\frac{q}{1+c e^{-q t}} \quad \text { with } \quad c:=\frac{q-u_{0}}{u_{0}} e^{q t_{0}}<0 \quad \text { on }\left(-\frac{1}{q} \ln \left(-\frac{1}{c}\right), t_{0}\right]
$$

and we have $u(t) \geq z(t)$ as long as $u$ and $z$ exist. This implies b) and e). 
e) $\rightarrow$ c) is trivial.

Now we prove that $v(t) \downarrow 0$ for $t \downarrow T_{0}$. With $t_{0}$ as in $\mathrm{d}$ ) it is clear that $\varphi(t) \in W_{+} \cap S_{-}(t)$ (see Section 2.6) and hence $\dot{v}>0$ on $\left(T_{0}, t_{0}\right]$. Assume $\lim _{t \rightarrow T_{0}} v(t)=\bar{v}>0$. Then

$$
\dot{v}(t)=v(t)(-1+u(t)+v(t))>v(t)(q-1+v(t))>\bar{v}(q-1+\bar{v})>0
$$

for $\left(T_{0}, t_{0}\right]$ and this contradicts $\bar{v}>0$.

\section{E-solutions}

For $p, q>1$ we consider solutions $\phi$ of $\left(\mathrm{M}_{p, \lambda}\right)$ on $(0, R), 0<R \leq \infty$, and their corresponding solutions $\varphi$ of $\left(\mathrm{MS}_{p, \lambda}\right)$ on $(-\infty, T), T:=\ln R$. Let $K$ be defined by (2.2.3). Also, let $\hat{v}(u):=v(t(u))$, where $t=t(u)$ is the inverse function of $u=u(t)$ in a neighborhood of $P_{3}$ (where $\dot{u}<0$ ). In the following theorem we characterize the $E$-solutions, from which also their existence follows.

Theorem 4.1. Let $p, q>1$. The following conditions are equivalent:

a) $\phi$ is an E-solution.

b) There exists some $\alpha>0$ such that

$$
\left.\begin{array}{l}
\phi(r)=\alpha-\frac{\alpha^{p}}{\lambda(\lambda+1)} r^{\lambda}[1+o(1)], \\
\phi^{\prime}(r)=-\frac{\alpha^{p}}{\lambda+1} r^{\lambda-1}[1+o(1)](r \rightarrow 0) .
\end{array}\right\}
$$

c) $r^{\lambda-1} \phi^{p}$ is integrable at 0 , which implies that there exists $\alpha>0$ such that

$$
\left.\begin{array}{l}
\phi(r)=\alpha-\int_{0}^{r}\left(1-\frac{s}{r}\right) s K(s) \phi^{p}(s) d s, r>0, \\
\phi^{\prime}(r)=-\frac{1}{r^{2}} \int_{0}^{r} s^{2} K(s) \phi^{p}(s) d s, r>0 .
\end{array}\right\}
$$

d) There exists $\alpha>0$ such that for $r \rightarrow 0$

$$
\begin{aligned}
\phi(r) & =\alpha-\frac{\alpha^{p}}{\lambda(\lambda+1)} r^{\lambda}+\frac{p \alpha^{2 p-1}}{2 \lambda^{2}(\lambda+1)(2 \lambda+1)} r^{2 \lambda} \\
& +\frac{\lambda \alpha^{p}}{2(\lambda+2)(\lambda+3)} r^{\lambda+2}+O\left(r^{\lambda+\min \{4,2 \lambda\}}\right), \\
\phi^{\prime}(r) & =-\frac{\alpha^{p}}{(\lambda+1)} r^{\lambda-1}+\frac{p \alpha^{2 p-1}}{\lambda(\lambda+1)(2 \lambda+1)} r^{2 \lambda-1} \\
& +\frac{\lambda \alpha^{p}}{2(\lambda+3)} r^{\lambda+1}+O\left(r^{\lambda-1+\min \{4,2 \lambda\}}\right) .
\end{aligned}
$$

e) There exists $\alpha>0$ such that

$$
\begin{aligned}
u(t)= & \lambda+1-\frac{p \alpha^{p-1}}{2 \lambda+1} e^{\lambda t}-\frac{\lambda(\lambda+1)}{(\lambda+3)} e^{2 t}+O\left(e^{\min \{4,2 \lambda\} t}\right), \\
v(t)= & \frac{\alpha^{p-1}}{(\lambda+1)} e^{\lambda t}\left[1-\frac{[p(\lambda+1)-(2 \lambda+1)] \alpha^{p-1}}{\lambda(\lambda+1)(2 \lambda+1)} e^{\lambda t}\right. \\
& \left.-\frac{\lambda(\lambda+1)}{2(\lambda+3)} e^{2 t}+O\left(e^{\min \{4,2 \lambda\} t}\right)\right]
\end{aligned}
$$


f) $\varphi(t) \rightarrow P_{3}^{-}(t \rightarrow-\infty)$.

In this case

$$
\phi(0):=\lim _{r \rightarrow 0} \phi(r)=\alpha,
$$

and

$$
\lim _{u \rightarrow q} \hat{v}^{\prime}(u)=\hat{v}^{\prime}(q)=\left\{\begin{array}{c}
-\frac{2 \lambda+1}{p(\lambda+1)}, \text { if } \lambda<2, \\
-\frac{5 \alpha^{p-1}}{3\left(p \alpha^{p-1}+6\right)}, \text { if } \lambda=2, \\
0, \text { if } \lambda>2 .
\end{array}\right.
$$

Proof. The existence of $E$-solutions follows from the possibility to solve the initial-value problem locally with $\phi(0)=\alpha$; this follows from Lemma 2.1. If we let

$$
L_{o \alpha}(r)=p K(r)|\alpha+\delta|^{p-1}
$$

then

$$
\frac{1}{r^{\lambda+1}} \int_{0}^{r} s^{2} L_{0 \alpha}(s) d s
$$

is bounded near zero and the same lemma implies

$$
r^{1-\lambda} \phi^{\prime}(r)-\frac{1}{r^{\lambda+1}} \int_{0}^{r} s^{2} K(s) \alpha^{p} d s \rightarrow 0 \quad(r \rightarrow 0) .
$$

L'Hospital's rule gives

$$
\lim _{r \rightarrow 0} \frac{1}{r^{\lambda+1}} \int_{0}^{r} s^{2} K(s) \alpha^{p} d s=\frac{\alpha^{p}}{\lambda+1} .
$$

Hence a) implies the second equation of b), and the first follows by integration.

a) $\Leftrightarrow \mathrm{c}) \Leftrightarrow \mathrm{b}$ ) are straightforward (see also $[24$, p. 8$]$ ).

b) $\Rightarrow$ d): We will stepwise improve the asymptotics. We begin with (4.1) and have

$$
\phi(r)=\alpha+O\left(r^{\lambda}\right)(r \rightarrow 0) .
$$

Using

$$
\begin{gathered}
(\alpha+x)^{p}=\alpha^{p}+p \alpha^{p-1} x+\frac{p(p-1) \alpha^{p-2}}{2} x^{2}+O\left(x^{3}\right),(x \rightarrow 0) \\
\frac{1}{\left(1+r^{2}\right)^{\lambda / 2}}=1-\frac{\lambda}{2} r^{2}+O\left(r^{4}\right),(r \rightarrow 0)
\end{gathered}
$$

and (4.2), we get

$$
\begin{aligned}
r^{2} \phi^{\prime}(r) & =-\int_{0}^{r} s^{2} K(s) \phi^{p}(s) d s \\
& =-\int_{0}^{r} s^{\lambda}\left[1+O\left(s^{2}\right)\right]\left[\alpha^{p}+O\left(s^{\lambda}\right] d s\right. \\
& =-\int_{0}^{r} s^{\lambda}\left[\alpha^{p}+O\left(s^{\min \{2, \lambda\}}\right)\right] d s \\
& =-\frac{\alpha^{p}}{(\lambda+1)} r^{\lambda+1}+O\left(r^{\min \{\lambda+3,2 \lambda+1\}}\right),
\end{aligned}
$$




$$
\left.\begin{array}{l}
\phi^{\prime}(r)=-\frac{\alpha^{p}}{(\lambda+1)} r^{\lambda-1}+O\left(r^{\min \{\lambda+1,2 \lambda-1\}}\right), \\
\phi(r)=\alpha-\frac{\alpha^{p}}{\lambda(\lambda+1)} r^{\lambda}+O\left(r^{\min \{\lambda+2,2 \lambda\}}\right) .
\end{array}\right\}
$$

Inserting (4.3) again into (4.2) we obtain

$$
\begin{aligned}
& r^{2} \phi^{\prime}(r) \\
& =-\int_{0}^{r} s^{2} K(s) \phi^{p}(s) d s \\
& =-\int_{0}^{r} s^{\lambda}\left[1-\frac{\lambda}{2} s^{2}+O\left(s^{4}\right)\right]\left[\alpha^{p}+p \alpha^{p-1}\left(-\frac{\alpha^{p}}{\lambda(\lambda+1)} s^{\lambda}+O\left(s^{\min \{\lambda+2,2 \lambda\}}\right)\right)+O\left(s^{2 \lambda}\right)\right] d s \\
& =-\int_{0}^{r} s^{\lambda}\left[\alpha^{p}-\frac{p \alpha^{2 p-1}}{\lambda(\lambda+1)} s^{\lambda}-\frac{\lambda \alpha^{p}}{2} s^{2}+O\left(s^{\min \{4,2 \lambda\}}\right)\right] d s \\
& =-\frac{\alpha^{p}}{(\lambda+1)} r^{\lambda+1}+\frac{p \alpha^{2 p-1}}{\lambda(\lambda+1)(2 \lambda+1)} r^{2 \lambda+1}+\frac{\lambda \alpha^{p}}{2(\lambda+3)} r^{\lambda+3}+O\left(r^{\min \{\lambda+5,3 \lambda+1\}}\right),
\end{aligned}
$$

and the equation for $\phi$ follows by integration (note that $\min \{4, \lambda+$ $2,2 \lambda\}=\min \{4,2 \lambda\})$.

d) $\Rightarrow$ e), f) : we have

$$
\begin{aligned}
& u(t)= \frac{r^{\lambda-1}}{\left(1+r^{2}\right)^{\lambda / 2}} \frac{\phi(r)^{p}}{-\phi^{\prime}(r)} \\
&= \frac{r^{\lambda-1} \alpha^{p}\left[1-\frac{\alpha^{p-1}}{\lambda(\lambda+1)} r^{\lambda}+O\left(r^{\min \{\lambda+2,2 \lambda\}}\right)\right]^{p}}{\left(1+r^{2}\right)^{\lambda / 2} \frac{\alpha^{p}}{(\lambda+1)} r^{\lambda-1}\left[1-\frac{p \alpha^{p-1}}{\lambda(2 \lambda+1)} r^{\lambda}-\frac{\lambda(\lambda+1)}{2(\lambda+3)} r^{2}+O\left(r^{\min \{4,2 \lambda\}}\right)\right]} \\
&= {\left[\lambda+1-\frac{p \alpha^{p-1}}{\lambda} r^{\lambda}+O\left(r^{\min \{\lambda+2,2 \lambda\}}\right)\right]\left[1-\frac{\lambda}{2} r^{2}+O\left(r^{4}\right)\right] . } \\
& \times\left[1+\frac{p \alpha^{p-1}}{\lambda(2 \lambda+1)} r^{\lambda}+\frac{\lambda(\lambda+1)}{2(\lambda+3)} r^{2}+O\left(r^{\min \{4,2 \lambda\}}\right)\right] \\
&=\lambda+1-\frac{p \alpha^{p-1}}{2 \lambda+1} r^{\lambda}-\frac{\lambda(\lambda+1)}{(\lambda+3)} r^{2}+O\left(r^{\min \{4,2 \lambda\}}\right), r=e^{t}
\end{aligned}
$$

while we also have

$v(t)$

$$
\begin{aligned}
= & r \frac{-\phi^{\prime}(r)}{\phi(r)}=r \frac{\frac{\alpha^{p}}{(\lambda+1)} r^{\lambda-1}\left[1-\frac{p \alpha^{p-1}}{\lambda(2 \lambda+1)} r^{\lambda}-\frac{\lambda(\lambda+1)}{2(\lambda+3)} r^{2}+O\left(r^{\min \{4,2 \lambda\}}\right)\right]}{\alpha-\frac{\alpha^{p}}{\lambda(\lambda+1)} r^{\lambda}+O\left(r^{\min \{\lambda+2,2 \lambda\}}\right)} \\
= & \frac{\alpha^{p-1}}{(\lambda+1)} r^{\lambda}\left[1-\frac{p \alpha^{p-1}}{\lambda(2 \lambda+1)} r^{\lambda}-\frac{\lambda(\lambda+1)}{2(\lambda+3)} r^{2}+O\left(r^{\min \{4,2 \lambda\}}\right)\right] . \\
& \times\left[1+\frac{\alpha^{p-1}}{\lambda(\lambda+1)} r^{\lambda}+O\left(r^{\min \{\lambda+2,2 \lambda\}}\right)\right] \\
= & \frac{\alpha^{p-1}}{(\lambda+1)} r^{\lambda}\left[1-\frac{[p(\lambda+1)-(2 \lambda+1)] \alpha^{p-1}}{\lambda(\lambda+1)(2 \lambda+1)} r^{\lambda}-\frac{\lambda(\lambda+1)}{2(\lambda+3)} r^{2}+O\left(r^{\min \{4,2 \lambda\}}\right)\right],
\end{aligned}
$$


$r=e^{t}$ which is e), and f) follows.

f) $\Rightarrow$ a): We specialize (2.4.1) to $\bar{u}:=u-q, \bar{v}=v$ and have

$$
\left.\begin{array}{l}
\dot{\bar{u}}=-q \bar{u}-p q v-\bar{u}^{2}-p \bar{u} v-\lambda(\bar{u}+q) g(t), \quad g(t):=\frac{e^{2 t}}{1+e^{2 t}} \\
\dot{v}=v(\lambda+\bar{u}+v\}) .
\end{array}\right\}
$$

Let $\varepsilon(t):=\bar{u}(t)+v(t) \rightarrow 0$. Then, for some $t_{0}$

$$
v(t)=v\left(t_{0}\right) e^{-\lambda t_{0}} \cdot e^{\lambda t+\int_{t_{0}}^{t} \varepsilon(s) d s} .
$$

If $|\varepsilon(t)| \leq \delta<\lambda$ for $t \leq t_{0}$, then

$$
v(t) \leq A_{0} e^{(\lambda-\delta) t}, A_{0}:=v\left(t_{0}\right) e^{-(\lambda-\delta) t_{0}} .
$$

If $\varepsilon$ is integrable at $-\infty$, then for some $A>0$,

$$
v(t)=A e^{\lambda t} \cdot e^{\int_{-\infty}^{t} \varepsilon(s) d s}, t \leq t_{0} .
$$

We may assume $\frac{q}{2}-\bar{u} \geq 0$. Then

$$
\begin{aligned}
\frac{1}{2}\left(\bar{u}^{2}\right) & =-(q+\bar{u}) \bar{u}^{2}-p \bar{u}^{2} v-\lambda \bar{u}^{2} g(t)-q(p v+\lambda g(t)) \bar{u} \\
& \leq-(q+\bar{u}) \bar{u}^{2}+\frac{q}{2}\left((p v+\lambda g(t))^{2}+\bar{u}^{2}\right) \\
& \leq q\left(p^{2} v^{2}+\lambda^{2} g^{2}(t)\right) \\
\bar{u}^{2}(t) & \leq 2 q\left(p^{2} \int_{-\infty}^{t} v^{2}(s) d s+\lambda^{2} \int_{-\infty}^{t} g^{2}(s) d s\right) \\
& \leq 2 q\left(p^{2} A_{0}^{2} e^{2(\lambda-\delta) t}+\lambda^{2} e^{4 t}\right) \\
\bar{u}(t) & =O\left(e^{\min (2, \lambda-\delta) t}\right) .
\end{aligned}
$$

We have shown that $\varepsilon$ is integrable at $-\infty$. Hence with (4.5)

$$
\begin{aligned}
v(t) & =A e^{\lambda t}\left[1+O\left(\int_{-\infty}^{t}(\bar{u}(s)+v(s)) d s\right)\right] \\
& =A e^{\lambda t}\left[1+O\left(e^{\min (2, \lambda) t}\right)\right] .
\end{aligned}
$$

Together with (4.6) we get $\bar{u}(t)=O\left(e^{\min (2, \lambda) t}\right)$,

$$
\begin{aligned}
\dot{\bar{u}} & =-(\lambda+1) \bar{u}-\lambda(\lambda+1) e^{2 t}-p(\lambda+1) A e^{\lambda t}+O\left(e^{\min \{4,2 \lambda\} t}\right), \\
{\left[e^{(\lambda+1) t} \bar{u}\right]^{\cdot} } & =-\lambda(\lambda+1) e^{(\lambda+3) t}-p(\lambda+1) A e^{(2 \lambda+1) t}+O\left(e^{\min \{\lambda+5,3 \lambda+1\} t}\right), \\
\bar{u}(t) & =-\frac{\lambda(\lambda+1)}{\lambda+3} e^{2 t}-\frac{p(\lambda+1)}{2 \lambda+1} A e^{\lambda t}+O\left(e^{\min \{4,2 \lambda\} t}\right), \\
u(t) & =\lambda+1-\frac{\lambda(\lambda+1)}{\lambda+3} e^{2 t}-\frac{p(\lambda+1)}{2 \lambda+1} A e^{\lambda t}+O\left(e^{\min \{4,2 \lambda\} t}\right),
\end{aligned}
$$


and with (4.5).

$v(t)=A e^{\lambda t}\left[1-\frac{\lambda(\lambda+1)}{2(\lambda+3)} e^{2 t}-\frac{p(\lambda+1)-(2 \lambda+1)}{\lambda(2 \lambda+1)} A e^{\lambda t}+O\left(e^{\min \{4,2 \lambda\} t}\right)\right]$.

The asymptotics for $u, v$ imply with (2.2.5)

$$
\begin{aligned}
\phi^{p-1}(r) & =\left(1+r^{2}\right)^{\lambda / 2} r^{-\lambda} u(\ln r) v(\ln r) \\
& \rightarrow q A=\alpha^{p-1}(r \rightarrow 0)
\end{aligned}
$$

This shows a).

We still have to show the last statement:

$$
\begin{aligned}
& \hat{v}^{\prime}(q)=\lim _{t \rightarrow-\infty} \frac{v(t)}{u(t)-q} \\
& =\lim _{t \rightarrow-\infty} \frac{\frac{\alpha^{p-1}}{(\lambda+1)} e^{\lambda t}\left[1-\frac{[p(\lambda+1)-(2 \lambda+1)] \alpha^{p-1}}{\lambda(\lambda+1)(2 \lambda+1)} e^{\lambda t}-\frac{\lambda(\lambda+1)}{2(\lambda+3)} e^{2 t}+O\left(e^{\min \{4,2 \lambda\} t}\right)\right]}{-\frac{p \alpha^{p-1}}{2 \lambda+1} e^{\lambda t}-\frac{\lambda(\lambda+1)}{(\lambda+3)} e^{2 t}+O\left(e^{\min \{4,2 \lambda\} t}\right)} \\
& =\left\{\begin{array}{c}
-\frac{2 \lambda+1}{p(\lambda+1)}, \text { if } \lambda<2, \\
-\frac{5 \alpha^{p-1}}{3\left(p \alpha^{p-1}+6\right)}, \text { if } \lambda=2, \\
0, \text { if } \lambda>2 .
\end{array}\right.
\end{aligned}
$$

On the other hand

$$
\begin{aligned}
& \lim _{u \rightarrow q} \hat{v}^{\prime}(u)=\lim _{t \rightarrow-\infty} \frac{v(-1+u+v)}{u(q(t)-u-p v)} \\
& =\frac{\lambda}{\lambda+1} \lim _{t \rightarrow-\infty} \frac{v}{q(t)-u-p v}=\frac{\lambda}{\lambda+1} \lim _{t \rightarrow-\infty} \frac{1}{\frac{q(t)-u}{v}-p}, \\
& \frac{q(t)-u}{v}=\frac{q-\lambda g(t)-u}{v} \\
& =\frac{\lambda+1-\lambda e^{2 t}-\left[\lambda+1-\frac{p \alpha^{p-1}}{2 \lambda+1} e^{\lambda t}-\frac{\lambda(\lambda+1)}{(\lambda+3)} e^{2 t}\right]+O\left(e^{\min \{4,2 \lambda\} t}\right)}{\frac{\alpha^{p-1}}{(\lambda+1)} e^{\lambda t}\left[1+O\left(e^{\min \{2, \lambda\} t}\right)\right]} \\
& =\frac{\frac{p \alpha^{p-1}}{2 \lambda+1} e^{\lambda t}-\frac{2 \lambda}{\lambda+3} e^{2 t}+O\left(e^{\min \{4,2 \lambda\} t}\right)}{\frac{\alpha^{p-1}}{(\lambda+1)} e^{\lambda t}\left[1+O\left(e^{\min \{2, \lambda\} t}\right)\right]} \rightarrow\left\{\begin{array}{c}
\frac{p(\lambda+1)}{2 \lambda+1}, \text { if } \lambda<2, \\
\frac{3\left(p \alpha^{p-1}-4\right)}{5 \alpha^{p-1}}, \text { if } \lambda=2, \\
-\infty, \text { if } \lambda>2,
\end{array}\right.
\end{aligned}
$$

so that

$$
\lim _{u \rightarrow q} \hat{v}^{\prime}(u)=\frac{\lambda}{\lambda+1} \lim _{t \rightarrow-\infty} \frac{1}{\frac{q(t)-u}{v}-p}=\hat{v}^{\prime}(q)
$$




\section{5. $M$-solutions}

In this section we let $p, q>1$, and consider solutions $\phi$ on $(0, R), 0<$ $R \leq \infty$, and the corresponding $\varphi$ on $(-\infty, T), T:=\ln R$. Our aim is the characterization and description of the $M$-solutions. We have to distinguish between the four different cases: $q>p$ (with the three subcases, $q>p+1$, $q=p+1, p<q<p+1), q=p, q<p$ (but $q \neq \frac{p+1}{2}$ ), and $q=\frac{p+1}{2}$.

\subsection{The case $q>p$}

In this section we show that the $M$-solutions are characterized by the property that their $\varphi(t)$ tend to $P_{2}$ as $t \rightarrow-\infty$. Though the existence of such solutions will follow later, the asymptotical stability of $P_{2}$ for $t \rightarrow-\infty$ follows from specializing the general linearization (2.4.1) to $P_{2}$ :

$$
\left(\begin{array}{c}
u \\
\bar{v}
\end{array}\right)=A\left(\begin{array}{c}
u \\
\bar{v}
\end{array}\right)+\left(\begin{array}{c}
-u^{2}-p u \bar{v} \\
u \bar{v}+\bar{v}^{2}
\end{array}\right)+\left(\begin{array}{c}
-(q-1) \frac{e^{2 t}}{1+e^{2 t}} u \\
0
\end{array}\right)
$$

with

$$
A:=\left(\begin{array}{cr}
q-p & 0 \\
1 & 1
\end{array}\right), \quad \bar{v}=v-1
$$

$A$ has the two positive eigenvalues $\lambda_{1}=q-p>0, \lambda_{2}=1$. It is possible to extend the classical theorem for asymptotic stability to this case where a time-dependent perturbation with the estimate $c \cdot e^{2 t}|u|$ appears. We omit the details. Hence any solution $\varphi$ starting in a small neighborhood of $P_{2}$ with a sufficiently negative $t_{0}$ will converge to $P_{2}$ as $t \rightarrow-\infty$.

Theorem 5.1. Let $q>p$. The following conditions are equivalent:

a) $\varphi$ is an $M$-solution.

b) There exists $c>0$ such that

$$
\left.\begin{array}{l}
\phi(r)=\frac{c}{r}[1+o(1)], \\
\phi^{\prime}(r)=-\frac{c}{r^{2}}[1+o(1)],(r \rightarrow 0) .
\end{array}\right\}
$$

c) There exists $c>0$ such that

$$
\begin{aligned}
u(t) & =c^{p-1} e^{(q-p) t}[1+o(1)], \\
v(t)-1 & =o(1)(t \rightarrow-\infty) .
\end{aligned}
$$

d) $\varphi(t) \rightarrow P_{2}(t \rightarrow-\infty)$.

In this case

$$
r^{2} \phi^{\prime}=-c-\int_{0}^{r} s^{2} K(s) \phi^{p} d s,
$$

and $c$ is uniquely determined. 
Proof. For a) $\rightarrow$ b) we extend arguments for $\left(\mathrm{EF}_{p, q}\right)$ [5, p.504] to our case. The function $z(t):=\phi\left(\frac{1}{t}\right)$ defined on $\left(\frac{1}{R}, \infty\right)$ is an unbounded solution of the equation

$$
z^{\prime \prime}(t)+f_{\lambda}(t) z^{p}=0, \text { where } f_{\lambda}(t):=\frac{1}{t^{2}\left(1+t^{2}\right)^{\lambda / 2}} .
$$

We multiply (5.1.3) with $z^{\prime}$ and integrate by parts from $t$ to $t_{0}>\frac{1}{R}$ :

$$
\frac{1}{2} z^{\prime}(t)^{2}+f_{\lambda}(t) \frac{z^{p+1}(t)}{p+1}-\int_{t_{0}}^{t} f_{\lambda}^{\prime}(s) \frac{z^{p+1}(s)}{p+1} d s=c_{1}+f_{\lambda}\left(t_{0}\right) \frac{z^{p+1}\left(t_{0}\right)}{p+1} .
$$

Because $f_{\lambda}^{\prime}<0, z^{\prime}$ is bounded, and there exists a constant $K \geq 0$ such that

$$
z(t) \leq K \cdot f_{\lambda}(t)^{-\frac{1}{p+1}} .
$$

Because for $t, \tau>t_{0}$ with (5.1.3)

$$
z^{\prime}(\tau)-z^{\prime}(t)=-\int_{t}^{\tau} f_{\sigma}(s) z^{p}(s) d s
$$

and

$$
f_{\lambda}(s) z^{p}(s) \leq K^{p} f_{\lambda}^{1-\frac{p}{p+1}}=K^{p}\left[t^{2}\left(1+t^{2}\right)^{\lambda / 2}\right]^{-\frac{1}{p+1}}
$$

is integrable $\left(-(2+\lambda) \frac{1}{p+1}<-1 \Longleftrightarrow 1+q>p+1 \Longleftrightarrow q>p\right)$, the limit $\lim _{t \rightarrow \infty} z^{\prime}(t)=: c$ exists. Because $z$ is unbounded, $c \geq 0$. Assume $c=0$. Then

We define

$$
z^{\prime}(t)=\int_{t}^{\infty} f_{\lambda}(s) z^{p}(s) d s .
$$

$$
r_{0}:=\inf \left\{r \in \mathbb{R} ; z^{\prime}(t)=O\left(t^{r}\right)\right\} .
$$

Because $z^{\prime}$ is bounded, we have $r_{0} \leq 0$. For $r>r_{0}$ we have with (5.1.4)

$$
z(t)=O\left(t^{r+1}\right) \quad \text { and } \quad z^{\prime}(t)=O\left(t^{-2-\lambda+p(r+1)+1}\right),
$$

hence $r_{0} \leq-2-\lambda+p\left(r_{0}+1\right)+1=p\left(r_{0}+1\right)-(\lambda+1) \Longleftrightarrow r_{0}+q \leq$ $p r_{0}+p \Longleftrightarrow q-p \leq(p-1) r_{0} \Longrightarrow r_{0}>0$, which is a contradiction. Hence $c>0$ and

$$
\begin{aligned}
z^{\prime}(t) & =c[1+o(1)] \quad(t \rightarrow-\infty), \\
r^{2} \cdot \phi^{\prime}(r) & =-c[1+o(1)] \quad(r \rightarrow 0),
\end{aligned}
$$

and b) follows.

b) $\rightarrow$ c): With (2.2.4) and (5.1.1)

$$
\begin{aligned}
& u(t)=\frac{r^{\lambda-1}}{\left(1+r^{2}\right)^{\lambda / 2}} \cdot \frac{\frac{c^{p}}{r^{p}}[1+o(1)]}{\frac{c}{r^{2}}[1+o(1)]}=c^{p-1} r^{\lambda+1-p}[1+o(1)] \longrightarrow 0, \\
& v(t)=r \frac{\frac{c}{r^{2}}[1+o(1)]}{\frac{c}{r}[1+o(1)]}=1+o(1), \quad r=e^{t},(t \rightarrow-\infty) .
\end{aligned}
$$


c) $\rightarrow$ d) is immediate.

d) $\rightarrow$ a): $\phi$ is an $M$ - or an $E$-solution. In the latter case, $\varphi(t) \rightarrow P_{3}^{-}$by Theorem 4.1, which is a contradiction to $\mathrm{d}$ ).

Finally, (5.1.3) follows by integration, because $r^{\lambda} \phi^{p}$ is integrable at 0 .

In the sequel we represent the $M$-solutions $\phi$ in the form

$$
\phi=S+\Theta
$$

where $S$ is a singular term of the form $S=\frac{c}{r} P(r)$ with an elementary explicitly given function $P$ of $r$ with $P(r)=1+o(1) \quad(r \rightarrow 0)$, while $\Theta$ is a (regular) solution of the initial value problem

$$
\left.\begin{array}{l}
\frac{1}{r^{2}}\left(r^{2} \Theta^{\prime}\right)^{\prime}=-K(r)(\Theta+S)^{p}-\frac{1}{r^{2}}\left(r^{2} S^{\prime}\right)^{\prime}, 0<r<R \\
\Theta(0):=\lim _{r \rightarrow 0} \Theta(r)=\beta \in \mathbb{R} .
\end{array}\right\}
$$

In terms of $P$, we have

$$
\begin{aligned}
\frac{1}{r^{2}}\left(r^{2} \Theta^{\prime}\right)^{\prime}= & -K(r)\left(\frac{c}{r} P+\Theta\right)^{p}-\frac{c}{r} P^{\prime \prime} \\
= & -K(r) \frac{c^{p}}{r^{p}} P^{p}\left(\left(1+\frac{r}{c P} \Theta\right)^{p}-1\right) \\
& +\left(-\frac{c}{r} P^{\prime \prime}-c^{p} r^{\mu-3} \frac{P^{p}}{\left(1+r^{2}\right)^{\lambda / 2}}\right) \\
= & : h_{1}(r, \Theta)+h_{2}(r) .
\end{aligned}
$$

Because

$$
K(r) \frac{c^{p}}{r^{p}} P^{p}(r)=O\left(r^{q-p-3}\right) \text { and }\left(1+\frac{r}{c P} \Theta\right)^{p}-1=O(r) \quad(r \rightarrow 0)
$$

we remark that $h_{1}(r, \Theta)$ satisfies the assumptions of Lemma 2.1. We are going to derive the different forms of $P$ and the asymptotic expansions for $\Theta$ in the subcases $q>p+1, q=p+1, p<q<p+1$ in the following three subsections.

5.1.1. The case $q>p+1$

Theorem 5.2. Let $q>p+1$. Then

a) Every $M$-solution $\phi$ has the form $\phi=S+\Theta$, where

$$
S(r)=\frac{c}{r}
$$

and $\Theta$ solves (5.1.6) and has the expansions

$$
\begin{aligned}
\Theta(r) & =\beta-\frac{c^{p}}{q-p}\left[\frac{1}{q-p-1} r^{q-p-1}+\frac{p \beta}{c(q-p+1)} r^{q-p}\right]+o\left(r^{q-p}\right) \\
\Theta^{\prime}(r) & =-c^{p}\left[\frac{1}{q-p} r^{q-p-2}+\frac{p \beta}{c(q-p+1)} r^{q-p-1}\right]+o\left(r^{q-p-1}\right)
\end{aligned}
$$

for some uniquely defined constants $c>0, \beta \in R$. 
b) Conversely, given any $c>0$ and $\beta \in \boldsymbol{R}$, there exists a unique solution $\Theta$ of (5.1.6) with $S(r):=\frac{c}{r}$ and $\phi:=S+\Theta$ (as long as $\phi$ is positive) is an $M$-solution.

In this case we have

$$
\left.\begin{array}{l}
\Theta(r)=\beta-\int_{0}^{r}\left(1-\frac{s}{r}\right) s K(s)\left[\frac{c}{s}+\Theta(s)\right]^{p} d s, 0<r<R, \\
u(t)=c^{p-1} e^{(q-p) t}\left[1+\frac{p \beta e^{t}}{c}+O\left(e^{\min \{2, q-p\} t}\right)\right], \\
v(t)=1-\frac{\beta e^{t}}{c}+\frac{c^{p-1} e^{(q-p) t}}{q-(p+1)}+O\left(e^{(q+1-p) t}\right), \quad(t \rightarrow-\infty),
\end{array}\right\}
$$

and

$$
\lim _{u \rightarrow 0+} \hat{v}^{\prime}(u)=\hat{v}^{\prime}(0)=\left\{\begin{array}{c}
\infty, \text { if } \beta<0, \\
\frac{1}{q-(p+1)}, \text { if } \beta=0, \\
-\infty, \text { if } \beta>0 .
\end{array}\right.
$$

Proof. a) Let $c>0$ be determined by Theorem 5.1. Then $\frac{1}{r^{2}} \int_{0}^{r} s^{2} K(s) \phi^{p} d s=$ $O\left(r^{\lambda-p-1}\right)$ is integrable because $\lambda-p>0 \Leftrightarrow q>p+1$. Hence, we have from (5.1.2) with $r_{0} \in(0, R)$

$$
\begin{aligned}
\phi(r) & =\frac{c}{r}-\frac{c}{r_{0}}+\phi\left(r_{0}\right)-\int_{r_{0}}^{r} \frac{d \sigma}{\sigma^{2}} \int_{0}^{\sigma} s^{2} K(s) \phi^{p}(s) d s, \\
& =: \frac{c}{r}+\beta-\int_{r_{0}}^{r} \frac{d \sigma}{\sigma^{2}} \int_{0}^{\sigma} s^{2} K(s) \phi^{p}(s) d s \\
& =: \frac{c}{r}+\Theta(r), 0<r<R .
\end{aligned}
$$

We define $S(r):=\frac{c}{r}$ and see that $\Theta$ satisfies (5.1.5) and (5.1.6) with $\frac{1}{r^{2}}\left(r^{2} S^{\prime}\right)^{\prime}=$ 0 . Hence (5.1.8) follows by partial integration. Any equation $\frac{c_{1}}{r}+\Theta_{1}(r)=$ $\frac{c_{2}}{r}+\Theta_{2}(r)$ implies $c_{1}=c_{2}$ and $\Theta_{1}(r)=\Theta_{2}(r)$, which shows the uniqueness of $c, \Theta$ and $\beta$. Now we remember $\Theta(r)=\beta+o(1)$ and have

$$
\begin{aligned}
\Theta^{\prime}(r) & =-\frac{1}{r^{2}} \int_{0}^{r} s^{2} K(s)\left[\frac{c}{s}+\Theta(s)\right]^{p} d s \\
& =-\frac{c^{p}}{r^{2}} \int_{0}^{r} s^{\lambda-p}\left(1+s^{2}\right)^{-\frac{\lambda}{2}}\left[1+\frac{s \Theta(s)}{c}\right]^{p} d s \\
& =-\frac{c^{p}}{r^{2}} \int_{0}^{r} s^{\lambda-p}\left[1+O\left(s^{2}\right)\right]\left[1+\frac{p \beta}{c} s+o(s)\right] d s \\
& =-\frac{c^{p}}{r^{2}} \int_{0}^{r} s^{\lambda-p}\left[1+\frac{p \beta}{c} s+o(s)\right] d s \\
& =-\frac{c^{p}}{r^{2}} \int_{0}^{r}\left[s^{\lambda-p}+\frac{p \beta}{c} s^{\lambda+1-p}+o\left(s^{\lambda+1-p}\right)\right] d s \\
& =-c^{p}\left[\frac{1}{\lambda+1-p} r^{\lambda-1-p}+\frac{p \beta}{c(\lambda+2-p)} r^{\lambda-p}\right]+o\left(r^{\lambda-p}\right)
\end{aligned}
$$


and the expansion for $\Theta$ follows by integration. This shows a). b) Given $c, \beta$, we define $S(r):=\frac{c}{r}$. Lemma 2.1 tells us that (5.1.6) (where $\left(r^{2} S^{\prime}\right)^{\prime}=0$ ) has a unique solution $\Theta$, and $\phi:=S+\Theta$ is an $M$-solution. Finally, using (2.2.4) and $\phi=S+\Theta$ we get

$u(t)$

$$
\begin{aligned}
& =\frac{r^{\lambda-1}}{\left(1+r^{2}\right)^{\lambda / 2}} \frac{\left[\frac{c}{r}+\beta-\frac{c^{p}}{\lambda+1-p}\left[\frac{1}{\lambda-p} r^{\lambda-p}+\frac{p \beta}{c(\lambda+2-p)} r^{\lambda+1-p}\right]+o\left(r^{\lambda+1-p}\right)\right]^{p}}{\frac{c}{r^{2}}+c^{p}\left[\frac{1}{\lambda+1-p} r^{\lambda-1-p}+\frac{p \beta}{c(\lambda+2-p)} r^{\lambda-p}\right]+o\left(r^{\lambda-p}\right)} \\
& =\frac{c^{p-1} r^{\lambda+1-p}}{\left(1+r^{2}\right)^{\lambda / 2}} \frac{\left[1+\frac{\beta r}{c}+O\left(r^{\lambda+1-p}\right)\right]^{p}}{1+O\left(r^{\lambda+1-p}\right)} \\
& =\frac{c^{p-1} r^{\lambda+1-p}}{1+O\left(r^{2}\right)} \frac{1+p\left[\frac{\beta r}{c}+O\left(r^{\lambda+1-p}\right)\right]+O\left(r^{2}\right)}{1+O\left(r^{\lambda+1-p}\right)} \\
& =c^{p-1} r^{\lambda+1-p} \frac{1+p \frac{\beta r}{c}+O\left(r^{\min \{2, \lambda+1-p\}}\right)}{1+O\left(r^{\min \{2, \lambda+1-p\}}\right)} \\
& =c^{p-1} r^{\lambda+1-p}\left[1+\frac{p \beta r}{c}+O\left(r^{\min \{2, \lambda+1-p\}}\right)\right]
\end{aligned}
$$

and similarly

$$
\begin{aligned}
v(t) & =r \frac{-\phi^{\prime}(r)}{\phi(r)} \\
& =r \frac{\frac{c}{r^{2}}+c^{p}\left[\frac{1}{\lambda+1-p} r^{\lambda-1-p}+\frac{p \beta}{c(\lambda+2-p)} r^{\lambda-p}\right]+o\left(r^{\lambda-p}\right)}{\frac{c}{r}+\beta-\frac{c^{p}}{\lambda+1-p}\left[\frac{1}{\lambda-p} r^{\lambda-p}+\frac{p \beta}{c(\lambda+2-p)} r^{\lambda+1-p}\right]+o\left(r^{\lambda+1-p}\right)} \\
& =\frac{1+c^{p-1} r^{\lambda+1-p}\left[\frac{1}{\lambda+1-p}+\frac{p \beta}{c(\lambda+2-p)} r\right]+o\left(r^{\lambda+2-p}\right)}{1+\frac{\beta r}{c}-\frac{c^{p-1} r^{\lambda+1-p}}{\lambda+1-p}\left[\frac{1}{\lambda-p}+\frac{p \beta}{c(\lambda+2-p)} r\right]+o\left(r^{\lambda+2-p}\right)} \\
& =1-\frac{\beta r}{c}+\frac{c^{p-1} r^{\lambda+1-p}}{\lambda-p}+O\left(r^{\lambda+2-p}\right) .
\end{aligned}
$$


Hence

$$
\begin{aligned}
\hat{v}^{\prime}(0) & =\lim _{t \rightarrow-\infty} \frac{v(t)-1}{u(t)} \\
& =\lim _{t \rightarrow-\infty} \frac{-\frac{\beta e^{t}}{c}+\frac{c^{p-1} e^{(\lambda+1-p) t}}{\lambda-p}+O\left(e^{(\lambda+2-p) t}\right)}{c^{p-1} e^{(\lambda+1-p) t}\left[1+\frac{p \beta e^{t}}{c}+O\left(e^{\min \{2, \lambda+1-p\} t}\right)\right]} \\
& =\left\{\begin{array}{l}
\infty, \text { if } \beta<0, \\
\frac{1}{\lambda-p}, \text { if } \beta=0, \\
-\infty, \text { if } \beta>0 .
\end{array}\right.
\end{aligned}
$$

and

$\lim _{u \rightarrow 0} \hat{v}^{\prime}(u)=\lim _{t \rightarrow-\infty} \frac{\dot{v}(t)}{\dot{u}(t)}=\lim _{t \rightarrow-\infty} \frac{v(-1+u+v)}{u(q(t)-u-p v)}=\frac{\hat{v}^{\prime}(0)+1}{q-p}=\hat{v}^{\prime}(0)$.

\subsubsection{The case $q=p+1$}

Theorem 5.3. Let $q=p+1$, i.e. $\lambda=p$. Then:

a) Every $M$-solution has the form $\phi=S+\Theta$, where

$$
S(r)=\frac{c}{r}-c^{p} \ln r
$$

and $\Theta$ solves (5.1.6) and has the expansions

$$
\begin{gathered}
\Theta(r)=\beta+\frac{p}{2} c^{2 p-1} r \ln r-\frac{p}{4} c^{p-1}\left(3 c^{p}+2 \beta\right) r+o(r) \\
\Theta^{\prime}(r)=\frac{p}{2} c^{2 p-1} \ln r-\frac{p}{4} c^{p-1}\left(3 c^{p}+2 \beta\right)+o(1), \quad(r \rightarrow 0)
\end{gathered}
$$

for some uniquely defined constants $c>0, \beta \in \mathbb{R}$.

b) Conversely, given any $c>0$ and $\beta \in \mathbb{R}$, there exists a unique solution $\Theta$ of (5.1.6) with $S$ given by (5.1.10), and $\phi:=S+\Theta$ is an $M$-solution (as long as $\phi$ is positive).

In this case $\Theta$ satisfies (5.1.7) with $P(r)=1-c^{p-1} r \ln r, P^{\prime \prime}(r)=$ $-\frac{c^{p-1}}{r}$, so that

$$
\begin{gathered}
h_{2}(r)=\frac{c^{p}}{r^{2}}\left(1-\frac{P^{p}(r)}{\left(1+r^{2}\right)^{p / 2}}\right)=\frac{p}{2} c^{p}(1+o(1)), \\
u(t)=c^{p-1} e^{t}\left[1-p c^{p-1} t e^{t}+O\left(e^{t}\right)\right] \\
v(t)=1+c^{p-1} t e^{t}+O\left(e^{t}\right)
\end{gathered}
$$

and

$$
\lim _{u \rightarrow 0+} \hat{v}^{\prime}(u)=\hat{v}^{\prime}(0)=-\infty
$$


Proof. a) Let $c>0$ be given by Theorem 5.1, which also gives

$$
\begin{aligned}
s^{2} K(s) \phi^{p}(s) & =\frac{s^{\lambda}}{\left(1+s^{2}\right)^{\lambda / 2}} \frac{c^{p}}{s^{p}}\left(\frac{s}{c} \phi(s)\right)^{p}=c^{p}\left(1+O\left(s^{2}\right)\right)(1+o(1))^{p} \\
& =c^{p}(1+o(1)), \\
\phi^{\prime}(r) & =-\frac{c}{r^{2}}-\frac{1}{r^{2}} \int_{0}^{r} s^{2} K(s) \phi^{p}(s) d s=-\frac{c}{r^{2}}-\frac{c^{p}}{r}+o\left(\frac{1}{r}\right), \\
\phi(r) & =\frac{c}{r}-c^{p} \ln r+o(\ln r)=\frac{c}{r}\left(1-c^{p-1} r \ln r+o(r \ln r)\right), \\
s^{2} K(s) \phi^{p}(s) & =c^{p}\left(1-p c^{p-1} s \ln s+o(s \ln s)\right), \\
\phi^{\prime}(r) & =-\frac{c}{r^{2}}-\frac{c^{p}}{r}+\frac{p}{2} c^{2 p-1} \ln r+o(\ln r), \\
\phi(r) & =\frac{c}{r}-c^{p} \ln r+\frac{p}{2} c^{2 p-1} r \ln r+o(r \ln r) .
\end{aligned}
$$

We define $S(r):=\frac{c}{r}-c^{p} \ln r$ and $\Theta(r):=\phi(r)-S(r)$. Then $\Theta$ satisfies the differential equation in (5.1.6), and because $\Theta^{\prime}=\phi^{\prime}-S^{\prime}$ is integrable, $\Theta(0)=: \beta$ exists. Again,

$$
\begin{aligned}
s^{2} K(s) \phi^{p}(s) & =s^{2} K(s) \frac{c^{p}}{s^{p}}\left(\frac{s}{c} \phi(s)\right)^{p} \\
& =c^{p}\left(1+O\left(s^{2}\right)\right)\left(1-c^{p-1} s \ln s+\frac{s}{c}(\beta+o(1))\right)^{p}, \\
\phi^{\prime}(r) & =-\frac{c}{r^{2}}-\frac{1}{r^{2}} \int_{0}^{r} s^{2} K(s) \phi^{p}(s) d s \\
& =-\frac{c}{r^{2}}-\frac{c^{p}}{r^{2}} \int_{0}^{r}\left(1-p c^{p-1} s \ln s+\frac{p \beta}{c} s+o(s)\right) d s \\
& =-\frac{c}{r^{2}}-\frac{c^{p}}{r}+\frac{p}{2} c^{2 p-1} \ln r-\frac{p}{4} c^{2 p-1}-\frac{p \beta}{2} c^{p-1}+o(1), \\
\Theta^{\prime}(r) & =\frac{p}{2} c^{2 p-1} \ln r-\frac{p}{4} c^{2 p-1}-\frac{p \beta}{2} c^{p-1}+o(1) .
\end{aligned}
$$

The expansion for $\Theta$ follows by integration and the uniqueness is shown as above. This shows a).

b) Given $c, \beta$, we define $S(r):=\frac{c}{r}-c^{p} \ln r=\frac{c}{r} P(r)$ and (5.1.11) follows. Together with the remark following (5.1.7) we see that the assumptions of Lemma 2.1 are satisfied, and (5.1.6) has a unique solution $\Theta$. Then $\phi:=S+\Theta$ is an $M$-solution.

Finally,

$u(t)$

$$
\begin{aligned}
& =\frac{r^{\lambda-1}}{\left(1+r^{2}\right)^{\lambda / 2}} \frac{\phi^{\lambda}(r)}{-\phi^{\prime}(r)}=\frac{r^{\lambda-1}}{\left(1+r^{2}\right)^{\lambda / 2}} \cdot \frac{\frac{c^{p}}{r^{p}}\left[1-c^{p-1} r \ln r+O(r)\right]^{p}}{-\frac{c}{r^{2}}\left[1-c^{p-1} r+\frac{p}{2} c^{p-2} r^{2} \ln r+O\left(r^{2} \ln r\right)\right]} \\
& =\frac{c^{p-1} r^{\lambda+1-p}}{\left(1+r^{2}\right)^{\lambda / 2}} \frac{\left[1-c^{p-1} r \ln r+O(r)\right]^{p}}{1-c^{p-1} r+\frac{p}{2} c^{p-2} r^{2} \ln r+O\left(r^{2} \ln r\right)} \\
& =c^{p-1} r\left[1-p c^{p-1} r \ln r+O(r)\right], r=e^{t}
\end{aligned}
$$


and

$$
\begin{aligned}
& v(t)=r \frac{-\phi^{\prime}(r)}{\phi(r)}=r \frac{\frac{c}{r^{2}}+c^{\lambda}\left[\frac{1}{r}-\frac{\lambda c^{\lambda-1}}{2} \ln r+\frac{\lambda\left(c^{\lambda}+2 \beta\right)}{4 c}\right]+o(1)}{\frac{c}{r}-c^{\lambda} \ln r+\beta r+\frac{p}{2} c^{2 p-1} r \ln r+O(r)} \\
& =\frac{1+c^{\lambda-1}\left[r-\frac{\lambda c^{\lambda-1}}{2} r^{2} \ln r+\frac{\lambda\left(c^{\lambda}+2 \beta\right)}{4 c} r^{2}\right]+o\left(r^{2}\right)}{1-c^{\lambda-1} r \ln r+\frac{\beta}{c} r+\frac{p}{2} c^{2 p-2} r^{2} \ln r+O\left(r^{2}\right)} \\
& =1+c^{\lambda-1} r \ln r+O(r),
\end{aligned}
$$

hence

$$
\begin{aligned}
\hat{v}^{\prime}(0) & =\lim _{t \rightarrow-\infty} \frac{v(t)-1}{u(t)} \\
& =\lim _{t \rightarrow-\infty} \frac{c^{\lambda-1} t e^{t}+O\left(e^{t}\right)}{c^{\lambda-1} e^{t}\left[1-\lambda c^{\lambda-1} t e^{t}+O\left(e^{t}\right)\right]} \\
& =-\infty .
\end{aligned}
$$

Because $\lim _{u \rightarrow 0} \hat{v}^{\prime}(u)=\frac{\hat{v}^{\prime}(0)+1}{q-p}$ as in (5.1.9) this shows (5.1.12).

\subsubsection{The case $p<q<p+1$}

Theorem 5.4. Let $p<q<p+1$. Let $\mu:=q-p \in(0,1)$ and $k_{0} \in \mathbb{N}$ such that $k_{0} \mu<1 \leq\left(k_{0}+1\right) \mu$. Then there exist constants $d_{1}:=\frac{1}{\mu(1-\mu)}, d_{2}, \ldots, d_{k_{0}+2}$ depending upon $p$ and $q$ with the following properties:

a) Every $M$-solution $\phi$ has the form $\phi=S+\Theta$, where

$$
S(r)=\left\{\begin{array}{r}
\frac{c}{r}\left[1+\sum_{i=1}^{k_{0}} d_{i} \rho^{i}\right], \rho:=c^{p-1} r^{\mu}, \\
\text { if }\left(k_{0}+1\right) \mu>1, \\
\frac{c}{r}\left[1+\sum_{i=1}^{k_{0}} d_{i} \rho^{i}+d_{k_{0}+1} c^{\left(k_{0}+1\right)(p-1)} r \ln r\right], \\
\text { if }\left(k_{0}+1\right) \mu=1,
\end{array}\right\}
$$

and $\Theta$ is a solution of (5.1.6) with the expansions

$$
\Theta(r)=\left\{\begin{aligned}
\beta+d_{k_{0}+1} c^{\left(k_{0}+1\right)(p-1)+1} r^{\left(k_{0}+1\right) \mu-1}+o\left(r^{\left(k_{0}+1\right) \mu-1}\right), & \text { if }\left(k_{0}+1\right) \mu>1, \\
\beta+d_{k_{0}+2} c^{\left(k_{0}+1\right)(p-1)+1} r^{\mu} \ln r+o\left(r^{\mu} \ln r\right), & \text { if }\left(k_{0}+1\right) \mu=1,
\end{aligned}\right.
$$




$$
\Theta^{\prime}(r)=\left\{\begin{aligned}
\left(\left(k_{0}+1\right) \mu-1\right) d_{k_{0}+1} c^{\left(k_{0}+1\right)(p-1)+1} r^{\left(k_{0}+1\right) \mu-2}+o\left(r^{\left(k_{0}+1\right) \mu-2}\right), & \text { if }\left(k_{0}+1\right) \mu>1, \\
\mu d_{k_{0}+2} c^{\left(k_{0}+1\right)(p-1)+1} r^{\mu-1} \ln r+o\left(r^{\mu-1} \ln r\right), & \text { if }\left(k_{0}+1\right) \mu=1,
\end{aligned}\right.
$$

for uniquely determined constants $c>0, \beta \in \mathbb{R}$.

b) Conversely, given any $c>0$ and $\beta \in \mathbb{R}$, there exists a unique solution $\Theta$ of (5.1.6) with $S$ given by (5.1.13) and $\phi:=S+\Theta$ is an $M$-solution (as long as $\phi$ is positive).

In this case $\Theta$ satisfies (5.1.7) with $P(r)=\frac{r}{c} S(r)$ and

$$
\begin{aligned}
& h_{2}(r)=\left\{\begin{array}{l}
O\left(r^{\left(k_{0}+1\right) \mu-3}\right), \text { if }\left(k_{0}+1\right) \mu>1, \\
O\left(r^{\mu-2} \ln r\right), \text { if }\left(k_{0}+1\right) \mu=1,
\end{array}\right. \\
& u(t)=c^{p-1} e^{\mu t}\left[1+\frac{q-1}{\mu(1-\mu)} c^{p-1} e^{\mu t}\right]+o\left(e^{2 \mu t}\right), \\
& v(t)=1-\frac{1}{\mu} c^{p-1} e^{\mu t}+o\left(e^{\mu t}\right),
\end{aligned}
$$

and

$$
\lim _{u \rightarrow 0} \hat{v}^{\prime}(u)=\hat{v}^{\prime}(0)=-\frac{1}{1-\mu} .
$$

Proof. a) Let $c$ be determined by Theorem 5.1, which also gives

$$
\begin{aligned}
s^{2} K(s) \phi^{p}(s) & =c^{p} s^{\mu-1}(1+o(1)), \\
\phi^{\prime}(r) & =-\frac{c}{r^{2}}-\frac{c^{p}}{\mu} r^{\mu-2}+o\left(r^{\mu-2}\right), \\
\phi(r) & =\frac{c}{r}-\frac{c^{p}}{\mu(\mu-1)} r^{\mu-1}+o\left(r^{\mu-1}\right)+A \\
& =\frac{c}{r}+\frac{c^{p}}{\mu(1-\mu)} r^{\mu-1}+o\left(r^{\mu-1}\right)=\frac{c}{r}\left[1+d_{1} c^{p-1} r^{\mu}+o\left(r^{\mu}\right)\right]
\end{aligned}
$$

(see the proof of a) of Theorem 5.3), because $\mu<1$. More generally, assume

$$
\phi(r)=\frac{c}{r}\left[1+d_{1} c^{p-1} r^{\mu}+\cdots+d_{k}\left(c^{p-1} r^{\mu}\right)^{k}+o\left(r^{k \mu}\right)\right]
$$

with constants $d_{i}=d_{i}(p, q), i=1, \ldots, k$, where $k \mu<1$. Let us introduce the notation

$$
\Sigma:=d_{1} \rho+\cdots+d_{k} \rho^{k}, \sigma:=\Sigma+o\left(\rho^{k}\right) .
$$

Then for $j \in \mathbb{N}$,

$$
\Sigma^{j}=O\left(\rho^{j}\right), \sigma^{j}=\sum_{l=0}^{j}\left(\begin{array}{l}
j \\
l
\end{array}\right) \Sigma^{j-l} O\left(\rho^{\ell k}\right)=\Sigma^{j}+o\left(\rho^{k}\right),
$$


so that

$$
\begin{aligned}
(1+\sigma)^{p} & =1+p \sigma+\cdots+\frac{p(p-1)(p-k+1)}{k !} \sigma^{k}+O\left(\sigma^{k+1}\right) \\
& =1+p \Sigma+\cdots+\frac{p(p-1)(p-k+1)}{k !} \Sigma^{k}+O\left(\rho^{k+1}\right)+o\left(\rho^{k}\right)+O\left(\Sigma^{k+1}\right) \\
& =1+\tilde{d}_{1} \rho+\cdots+\tilde{d}_{k} \rho^{k}+o\left(\rho^{k}\right)
\end{aligned}
$$

with appropriate definition of $\tilde{d}_{i}=\tilde{d}_{i}(p, q), i=1, \ldots, k$. Hence

$$
\begin{aligned}
s^{2} K(s) \phi^{p}(s) & =c^{p} s^{\mu-1}\left(1+O\left(s^{2}\right)\right)(1+\sigma)^{p} \\
& =c^{p} s^{\mu-1}\left[1+\sum_{i=1}^{k} \tilde{d}_{i} c^{i(p-1)} s^{i \mu}+o\left(s^{\mu k}\right)\right] \\
& =c\left[c^{p-1} s^{\mu-1}+\sum_{i=1}^{k} \tilde{d}_{i} c^{(i+1)(p-1)} s^{(i+1) \mu-1}+o\left(s^{(k+1) \mu-1}\right)\right], \\
\int_{0}^{r} s^{2} K(s) \phi^{p}(s) d s & =c\left[c^{p-1} \frac{r^{\mu}}{\mu}+\sum_{i=1}^{k} \tilde{d}_{i} c^{(i+1)(p-1)} \frac{r^{(i+1) \mu}}{(i+1) \mu}+o\left(s^{(k+1) \mu}\right)\right] \\
\phi^{\prime}(r) & =-\frac{c}{r^{2}}-c\left[\frac{1}{\mu} c^{p-1} r^{\mu-2}+\sum_{i=1}^{k} \frac{\tilde{d}_{i}}{(i+1) \mu} c^{(i+1)(p-1)} r^{(i+1) \mu-2}+o\left(r^{(k+1) \mu-2}\right)\right] \\
& =\quad-\frac{c}{r^{2}}-c\left[\frac{1}{\mu} c^{p-1} r^{\mu-2}+\sum_{i=2}^{k} \frac{\tilde{d}_{i-1}}{i \mu} c^{i(p-1)} r^{i \mu-2}\right. \\
& \left.\quad \frac{\tilde{d}_{k}}{(k+1) \mu} c^{(k+1)(p-1)} r^{(k+1) \mu-2}+o\left(r^{(k+1) \mu-2}\right)\right] .
\end{aligned}
$$

The first $k$ terms in the bracket are not integrable at 0 because $k \mu<1$. Integrating from $r_{0}>0$ to $r$ we get, with some $\beta \in \mathbb{R}$,

$$
\begin{aligned}
\phi(r) & =\frac{c}{r}+c\left[\frac{1}{\mu(1-\mu)} c^{p-1} r^{\mu-1}+\sum_{i=2}^{k} \frac{\tilde{d}_{i-1}}{i \mu(1-i \mu)} c^{i(p-1)} r^{i \mu-1}\right] \\
& +\beta-c \frac{\tilde{d}_{k}}{(k+1) \mu} c^{(k+1)(p-1)} \int_{r_{0}}^{r} s^{(k+1) \mu-2} d s+o\left(r^{(k+1) \mu-1}\right)
\end{aligned}
$$

If $(k+1) \mu<1$, we find $d_{i}=\frac{\tilde{d}_{i-1}}{i \mu(1-i \mu)}, i=2, \ldots, k$ and

$$
\phi(r)=\frac{c}{r}\left[1+d_{1} \rho+\cdots+d_{k} \rho^{k}+d_{k+1} \rho^{k+1}+o\left(r^{(k+1) \mu}\right)\right],
$$

where $d_{k+1}:=\frac{\tilde{d}_{k}}{(k+1) \mu(1-(k+1) \mu)}$, that is, (5.1.14) holds for $k+1$. By induction, we can repeat the process until the biggest $k$ with $k \mu<1$, which is $k_{0}$. Hence 
(5.1.14) also holds for $k_{0}$. If $\left(k_{0}+1\right) \mu>1$, we have for $k=k_{0}$

$$
\phi(r)=\frac{c}{r}\left[1+\sum_{i=1}^{k_{0}} d_{i} \rho^{i}\right]+\beta+d_{k_{0}+1} c^{\left(k_{0}+1\right)(p-1)+1} r^{\left(k_{0}+1\right) \mu}+o\left(r^{\left(k_{0}+1\right) \mu}\right) .
$$

The singular term $S$ and the regular term $\Theta$ can now be read off. In the case $\left(k_{0}+1\right) \mu=1$, we let $D=-\tilde{d}_{k_{0}} c^{\left(k_{0}+1\right)(p-1)+1}$ and we have

$$
\begin{aligned}
\phi(r) & =\frac{c}{r}\left[1+\sum_{i=1}^{k_{0}} d_{i} s^{i}\right]+\beta-\tilde{d}_{k_{0}} c^{\left(k_{0}+1\right)(p-1)+1}\left(\ln r-\ln r_{0}\right)+o(\ln r) \\
& =\frac{c}{r}\left[1+\sum_{i=1}^{k_{0}} d_{i} s^{i}+D \cdot r \ln r+o(r \ln r)\right] .
\end{aligned}
$$

We need a further iteration. We let

$$
\Sigma:=d_{1} \rho+\cdots+d_{k_{0}} \rho^{k_{0}}, L(r):=D r \ln r, \sigma:=\Sigma+L+o(r \ln r) .
$$

Then for $j \geq 2$,

$$
\begin{aligned}
\sigma^{j} & =\Sigma^{j}+\sum_{i=1}^{j-1}\left(\begin{array}{l}
j \\
i
\end{array}\right) \Sigma^{j-i}(L+o(r \ln r))^{i}+(L+o(r \ln r))^{j} \\
& =\Sigma^{j}+\sum_{i=1}^{j-1} O\left(\rho^{j-i}\right) L^{i}(1+o(1))+L^{j}(1+o(1))=\Sigma^{j}+o(r \ln r),
\end{aligned}
$$

so that

$$
\begin{aligned}
(1+\sigma)^{p}= & 1+p \sigma+\frac{p(p-1)}{2} \sigma^{2}+\cdots+\frac{p(p-1) \cdots\left(p-k_{0}+1\right)}{k !} \sigma^{k_{0}}+O\left(\sigma^{k_{0}+1}\right) \\
= & 1+p \Sigma+\frac{p(p-1)}{2} \Sigma^{2}+\cdots+\frac{p(p-1) \cdots\left(p-k_{0}+1\right)}{k !} \Sigma^{k_{0}}+p L \\
& +O\left(\Sigma^{k_{0}+1}\right)+o(r \ln r) \\
= & 1+\sum_{i=1}^{k_{0}} \tilde{d}_{i} \rho^{i}+O\left(\rho^{k_{0}+1}\right)+p L+O\left(\rho^{k_{0}+1}\right)+o(r \ln r) \\
= & 1+\sum_{i=1}^{k_{0}} \tilde{d}_{i} \rho^{i}+p L+o(r \ln r)
\end{aligned}
$$


for suitably chosen $\tilde{d}_{i}=\tilde{d}_{i}(p, q), i=1, \ldots, k_{0}$; note that $\rho^{k_{0}+1}=c^{\left(k_{0}+1\right)(p-1)} r$.

Hence as above,

$$
\begin{aligned}
s^{2} K(s) \phi^{p}(s) & =c^{p} s^{\mu-1}\left[1+\sum_{i=1}^{k_{0}} \tilde{d}_{i} c^{i(p-1)} s^{i \mu}+p D s \ln s+o(s \ln s)\right] \\
& =c\left[c^{p-1} s^{\mu-1}+\sum_{i=1}^{k_{0}} \tilde{d}_{i} c^{(i+1)(p-1)} s^{(i+1) \mu-1}+p D s^{\mu} \ln s+o\left(s^{\mu} \ln s\right)\right], \\
\phi^{\prime}(r) & =-\frac{c}{r^{2}}-c\left[\frac{1}{\mu} c^{p-1} r^{\mu-2}+\sum_{i=2}^{k_{0}} \frac{\tilde{d}_{i-1}}{i \mu} c^{i(p-1)} r^{i \mu-2}+\tilde{d}_{k_{0}} c^{\left(k_{0}+1\right)(p-1)} r^{-1}\right. \\
& \left.-\frac{p D}{\mu+1} r^{\mu-1} \ln r+o\left(r^{\mu-1} \ln r\right)\right] \\
\phi(r) & =\frac{c}{r}+c\left[\frac{1}{\mu(1-\mu)} c^{p-1} r^{\mu-1}+\sum_{i=2}^{k_{0}} \frac{\tilde{d}_{i-1}}{i \mu(1-i \mu)} c^{i(p-1)} r^{i \mu-1}+d_{k_{0}+1} c^{\left(k_{0}+1\right)(p-1)} \ln r\right] \\
& +\beta+\frac{p D}{\mu(\mu+1)} c^{\left(k_{0}+1\right)(p-1)+1} r^{\mu} \ln r+o\left(r^{\mu} \ln r\right) \\
& =\frac{c}{r}\left[1+\sum_{i=1}^{k_{0}} d_{i} \rho^{i}+d_{k_{0}+1} c^{\left(k_{0}+1\right)(p-1)} r \ln r\right] \\
& +\beta+d_{k_{0}+2} c^{\left(k_{0}+1\right)(p-1)+1} r^{\mu} \ln r+o\left(r^{\mu} \ln r\right),
\end{aligned}
$$

where $d_{k_{0}+1}:=\tilde{d}_{k_{0}}, d_{k_{0}+2}=\frac{-p \tilde{d}_{k_{0}}}{\mu(\mu+1)}$, and $S$ and $\Theta$ can be read off again.

This shows a).

b) With the constants given we define $S$ as in a) and $P(r):=\frac{r}{c} S(r)$. Let $\left(k_{0}+1\right) \mu=1$. Using the relation between the $d_{i}$ 's and the $\tilde{d}_{i}$ 's one easily shows

$$
P^{\prime \prime}(r)=-c^{p-1} r^{\mu+2}\left[1+\sum_{i=1}^{k_{0}} \tilde{d}_{i} \rho^{i}\right] .
$$

Furthermore, we remember

$$
P^{p}(r)=(1+\Sigma+L)^{p}=1+\sum_{i=1}^{k_{0}} \tilde{d}_{i} \rho^{i}+p L+o(r \ln r) .
$$

Hence with (5.1.7),

$$
\begin{aligned}
h_{2}(r) & =-\frac{c}{r} P^{\prime \prime}(r)-c^{p} \frac{r^{\mu-3}}{\left(1+r^{2}\right)^{\lambda / 2}} P^{p}(r) \\
& =-\frac{c}{r} P^{\prime \prime}(r)-c^{p} r^{\mu-3} P^{p}(r)+c^{p} r^{\mu-3} P^{p}(r)\left(1-\left(1+r^{2}\right)^{-\lambda / 2}\right) \\
& =c^{p} r^{\mu-3}\left[1+\sum_{i=1}^{k_{0}} \tilde{d}_{i} \rho^{i}-\left(1+\sum_{i=1}^{k_{0}} \tilde{d}_{i} \rho^{i}+p L+o(r \ln r)\right)\right]+O\left(r^{\mu-1}\right) \\
& =r^{\mu-3} \cdot O(r \ln r)=O\left(r^{\mu-2} \ln r\right) .
\end{aligned}
$$


The case $\left(k_{0}+1\right) \mu>1$ is similar. Hence $h_{2}$ satisfies the assumptions of Lemma 2.1, and together with the remark following (5.1.7) we see that there exists a unique solution $\Theta$ of (5.1.6).

Finally,

$$
\begin{aligned}
& u(t)=\frac{r^{\lambda-1}}{\left(1+r^{2}\right)^{\lambda / 2}} \frac{\phi(r)^{p}}{-\phi^{\prime}(r)} \\
& =\frac{r^{\lambda-1}}{\left(1+r^{2}\right)^{\lambda / 2}} \frac{\left[\frac { c } { r } \left(1+\frac{1}{r^{2}}\left[1+\frac{1}{\mu} c^{p-1} r^{\mu}+\ldots+\frac{d_{k_{0}}}{\left(k_{0}+1\right) \mu}\left(c^{p-1} r^{\mu}\right)^{k_{0}+1}+o\left(r^{\mu\left(k_{0}+1\right)}\right)\right]\right.\right.}{\left(1+r^{2}\right)^{\lambda / 2}} \frac{\left[1+\frac{1}{\mu(1-\mu)} c^{p-1} r^{\mu}+\ldots+c^{k_{0}(p-1)} d_{k_{0}} r^{k_{0} \mu}+o\left(r^{k_{0} \mu}\right)\right]^{p}}{c^{p-1} r^{\mu}+\ldots+\frac{d_{k_{0}}}{\left(k_{0}+1\right) \mu}\left(c^{p-1} r^{\mu}\right)^{k_{0}+1}+o\left(r^{\mu\left(k_{0}+1\right)}\right)} \\
& =\frac{c^{p-1} r^{q-p}}{\left(1+k^{k_{0}}+1\right)} d_{\left.k^{k_{0} \mu}+o\left(r^{k_{0} \mu}\right)\right)}^{p} \\
& =c^{p-1} r^{q-p}\left[1+\frac{q-1}{\mu(1-\mu)} c^{p-1} r^{\mu}+o\left(r^{\mu}\right)\right]
\end{aligned}
$$

and

$v(t)$

$$
\begin{aligned}
& =r \frac{-\phi^{\prime}(r)}{\phi(r)}=r \frac{\frac{c}{r^{2}}\left[1+\frac{1}{\mu} c^{p-1} r^{\mu}+\ldots+\frac{d_{k_{0}}}{\left(k_{0}+1\right) \mu}\left(c^{p-1} r^{\mu}\right)^{k_{0}+1}+o\left(r^{\mu\left(k_{0}+1\right)}\right)\right]}{\frac{c}{r}\left(1+\frac{1}{\mu(1-\mu)} c^{p-1} r^{\mu}+\ldots+c^{k_{0}(p-1)} d_{k_{0}} r^{k_{0} \mu}+o\left(r^{k_{0} \mu}\right)\right)} \\
& =\frac{1+\frac{1}{\mu} c^{p-1} r^{\mu}+\ldots+\frac{d_{k_{0}}}{1+\frac{1}{\mu(1-\mu)}}\left(c^{p-1} c^{p-1} r^{\mu}+\ldots+c^{k_{0}(p-1)} d_{k_{0}} r^{k_{0} \mu}+o\left(r^{k_{0} \mu}\right)\right.}{1-\frac{1}{1-\mu} c^{p-1} r^{\mu}+o\left(r^{\mu}\right) .} \\
& =1-
\end{aligned}
$$

Hence, with (5.1.9),

$$
\begin{aligned}
\hat{v}^{\prime}(0) & =\lim _{t \rightarrow-\infty} \frac{v(t)-1}{u(t)}=\lim _{t \rightarrow-\infty} \frac{-\frac{1}{1-\mu} c^{p-1} e^{\mu t}+o\left(e^{\mu t}\right)}{c^{p-1} e^{\mu t}\left[1+\frac{p+\mu-1}{\mu(1-\mu)} c^{p-1} e^{\mu t}+o\left(e^{\mu t}\right)\right]} \\
& =-\frac{1}{1-\mu}=\lim _{u \rightarrow 0} \hat{v}^{\prime}(\mu) \cdot \square
\end{aligned}
$$




\subsection{The case $q=p$}

There exist solutions $\varphi$ for which $\varphi(t) \rightarrow P_{2}(t \rightarrow-\infty)$. In fact, because $q>\frac{p+1}{2}$, by Lemma 2.3 , if $\varphi\left(t_{0}\right) \in G_{-}, \varphi(t) \in G_{-}$for $t \leq t_{0}$. By Thieme's theorem and the absence of Cases (B) and (C) for the limit system $\left(\operatorname{EFS}_{p, q}\right)[4$, case $n=m+3$ ], $\varphi$ must converge to a stationary point. It cannot converge to $P_{1}$ by Lemma 2.4 , and $P_{3}^{-} \notin \bar{G}_{-}$because $q>\frac{p+1}{2}$. Hence $\varphi$ converges to $P_{2}$.

Before proving the main theorem of this section we first establish the following lemmas.

Lemma 5.5. Consider the differential equation $y^{\prime}=r(t) y+b(t)$ on $\left(-\infty, t_{0}\right]$. If $r(t) \rightarrow r_{0}>0, b(t) \rightarrow b_{0} \in \mathbb{R}(t \rightarrow-\infty)$, then

$$
y(t) \rightarrow-\frac{b_{0}}{r_{0}} \quad(t \rightarrow-\infty) .
$$

Proof. We let $R(t)=\int_{t_{0}}^{t} r(s) d s$ and have

$$
\begin{aligned}
y(t) & =e^{R(t)}\left[y\left(t_{0}\right)-\int_{t}^{t_{0}} b(s) e^{-R(s)} d s\right] \\
& =e^{R(t)}\left[y\left(t_{0}\right)-\int_{t}^{t_{0}}\left(-r(s) \frac{b_{0}}{r_{0}}+b(s)\right) e^{-R(s)} d s\right]-\frac{b_{0}}{r_{0}}\left(1-e^{R(t)}\right), t \leq t_{0} .
\end{aligned}
$$

Given $\varepsilon>0$, there exists $t_{1}<t_{0}$ such that for $s \leq t_{1}$

$$
\left|-r(s) \frac{b_{0}}{r_{0}}+b(s)\right|<\frac{\varepsilon}{2} r_{0}<\varepsilon r(s) .
$$

For $t \leq t_{1}$ we write $\int_{t}^{t_{0}}=\int_{t}^{t_{1}}+\int_{t_{1}}^{t_{0}}$ and then have

$$
\begin{aligned}
\left|\int_{t}^{t_{1}}\left(-r(s) \frac{b_{0}}{r_{0}}+b(s)\right) e^{-R(s)} d s\right| & <\varepsilon \int_{t}^{t_{1}} r(s) e^{-R(s)} d s \\
& =\varepsilon\left(e^{-R(t)}-e^{-R\left(t_{1}\right)}\right)<\varepsilon e^{-R(t)}
\end{aligned}
$$

which shows $y(t) \rightarrow-\frac{b_{0}}{r_{0}}$ because $e^{R(t)} \rightarrow 0$.

Lemma 5.6. Consider the differential equation $y^{\prime}=r(t) y+b(t)$ on $\left(-\infty, t_{0}\right]$. If $r(t)=O\left((-t)^{-1-\delta}\right)$ for some $\delta>0, b(t) \rightarrow b_{0} \neq 0$ as $t \rightarrow-\infty$, then

$$
\frac{y(t)}{t} \rightarrow b_{0} \text { as } t \rightarrow-\infty
$$


Proof. We let $R(t):=\int_{t_{0}}^{t} r(s) d s=A+B(t)$, where $A:=\int_{t_{0}}^{-\infty} r(s) d s$ and $B(t):=\int_{-\infty}^{t} r(s) d s=O\left((-t)^{-\delta}\right)$. Then

$$
\begin{aligned}
\frac{y(t)}{t} & =\frac{e^{R(t)} y\left(t_{0}\right)}{t}+\frac{\int_{t_{0}}^{t} b(s) e^{R(t)-R(s)} d s}{t} \\
& =\frac{e^{R(t)} y\left(t_{0}\right)}{t}+e^{B(t)} \frac{\int_{t_{0}}^{t} b(s) e^{-B(s)} d s}{t} .
\end{aligned}
$$

It is clear that $\frac{e^{R(t)} y\left(t_{0}\right)}{t} \rightarrow 0, e^{B(t)} \rightarrow 1$, and $\int_{t_{0}}^{t} b(s) e^{-B(s)} d s \rightarrow-\operatorname{sign}\left(b_{0}\right) \infty$. Applying L'Hospital's rule then gives

$$
\lim _{t \rightarrow-\infty} \frac{y(t)}{t}=\lim _{t \rightarrow-\infty} b(t) e^{-B(t)}=b_{0}
$$

Remark 2. The above two lemmas can be easily extended to the case $t \rightarrow$ $\infty$. For example, in Lemma 5.5. we have to require $r_{0}<0$ in this case.

Theorem 5.7. Let $q=p$. The following conditions are equivalent:

a) $\phi$ is an $M$-solution.

b) We have

$$
\begin{aligned}
\phi(r) & =\frac{1}{\left(-\ln r^{\lambda}\right)^{1 / \lambda}} \cdot \frac{1}{r}(1+o(1)), \\
\phi^{\prime}(r) & =-\frac{1}{\left(-\ln r^{\lambda}\right)^{1 / \lambda}} \cdot \frac{1}{r^{2}}(1+o(1)) \quad(r \rightarrow 0) .
\end{aligned}
$$

c) We have

$$
\begin{aligned}
u(t) & =-\frac{1}{\lambda} \cdot \frac{1}{t}(1+o(1)), \\
v(t)-1 & =\frac{1}{\lambda} \cdot \frac{1}{t}(1+o(1)) \quad(t \rightarrow-\infty) .
\end{aligned}
$$

d) $\varphi(t) \rightarrow P_{2}(t \rightarrow-\infty)$.

In this case

$$
\lim _{u \rightarrow 0+} \hat{v}^{\prime}(u)=\hat{v}^{\prime}(0)=-1 .
$$

Proof. a) $\rightarrow$ d): By Theorem 3.1 on $F$-solutions, we conclude that $C^{-}(\varphi)$ is bounded. By Thieme's theorem and the absence of Cases (B) and (C), $\varphi$ must converge to a stationary point of $\left(\mathrm{EF}_{p, q}\right)[4$, case $n=m+3]$. The convergence to $P_{1}$ and $P_{3}^{-}$is not possible by Lemma 2.4 and Theorem 4.1 on $E$-solutions. Hence $\varphi(t) \rightarrow P_{2}$. 
d) $\rightarrow$ c): We define $y:=\frac{v-1}{u}$. Then

$$
\begin{aligned}
\dot{y} & =\frac{u \dot{v}-(v-1) \dot{u}}{u^{2}}=\frac{v(-1+u+v)}{u}-\frac{v-1}{u}(q(t)-u-p v) \\
& =v y+v-q(t) y+v-1+p v y \\
& =((p+1) v-q(t)) y+2 v-1
\end{aligned}
$$

where

$$
((p+1) v-q(t)) \rightarrow 1,2 v-1 \rightarrow 1(t \rightarrow-\infty) .
$$

This implies that $y(t) \rightarrow-1$ as $t \rightarrow-\infty$ by Lemma 5.5 . Now

$$
\begin{aligned}
\left(\frac{1}{u}\right)^{\cdot} & =-\frac{\dot{u}}{u^{2}}=-\frac{u(q(t)-u-p v)}{u^{2}}=-q(t) \frac{1}{u}+1+p \frac{v-1}{u}+p \frac{1}{u} \\
& =(q-q(t)) \frac{1}{u}+q y+1=: g(t) \frac{1}{u}+q y+1, \text { where } \\
g(t) & =\lambda \frac{e^{2 t}}{1+e^{2 t}}=O\left(e^{2 t}\right), q y+1 \rightarrow-q+1=-\lambda(t \rightarrow-\infty),
\end{aligned}
$$

which implies that $\frac{1}{t} \cdot \frac{1}{u(t)} \rightarrow-q+1=-\lambda$ as $t \rightarrow-\infty$ by Lemma 5.6. Hence

$$
\lim _{t \rightarrow-\infty} t(v(t)-1)=\lim _{t \rightarrow-\infty} t u(t) y(t)=\frac{1}{\lambda} .
$$

This shows c).

c) $\rightarrow$ b): c) means

$$
u(\ln r)=-\frac{1}{\lambda} \cdot \frac{1}{\ln r}(1+o(1))=-\frac{1}{\ln r^{\lambda}}(1+o(1)),
$$

Hence with (2.2.5)

$$
\begin{aligned}
\phi(r) & =\left(\left(1+r^{2}\right)^{\lambda / 2} \cdot r^{-\lambda} \cdot u(\ln r) \cdot v(\ln r)\right)^{\frac{1}{\lambda}} \quad(\lambda=p-1) \\
& =\frac{1}{r} \cdot\left(-\frac{1}{\ln r^{\lambda}}(1+o(1))\right)^{1 / \lambda}(1+o(1)) \\
& =\frac{1}{\left(-\ln r^{\lambda}\right)^{1 / \lambda}} \cdot \frac{1}{r}(1+o(1)) \\
\phi^{\prime}(r) & =-\frac{1}{r} \phi(r)(1+o(1)) .
\end{aligned}
$$

This shows b).

b) $\rightarrow$ a) is trivial.

Finally, (5.2.2) means

$$
\dot{y}=r(t) y+b(t), \text { with } r(t)=(p+1) v-q(t), b(t)=2 v-1,
$$

where c) implies

$$
r(t)=1+\frac{\lambda+2}{\lambda t}[1+o(1)], b(t)=1+\frac{2}{\lambda t}[1+o(1)] .
$$


Let us define $y_{1}(t):=t(y(t)+1)$. Then

$$
\begin{aligned}
\dot{y}_{1} & =\left[r(t)+\frac{1}{t}\right] y_{1}+t[b(t)-r(t)], \\
r(t)+\frac{1}{t} & \rightarrow 1, t[b(t)-r(t)] \rightarrow-1,
\end{aligned}
$$

so that again by Lemma 5.5 we have $y_{1} \rightarrow 1$ as $t \rightarrow-\infty$. Hence by c)

$$
\begin{aligned}
\lim _{u \rightarrow 0+} \hat{v}^{\prime}(u) & =\lim _{t \rightarrow-\infty} \frac{v(-1+u+v)}{u(q(t)-u-p v)}=\lim _{t \rightarrow-\infty} \frac{v\left(\frac{v-1}{u}+1\right)}{q(t)-p-u-p(v-1)} \\
& =\lim _{t \rightarrow-\infty} v \frac{y_{1}}{t(q(t)-p)-t u-p t(v-1)} \\
& =1 \cdot \frac{1}{0+\frac{1}{\lambda}-p \frac{1}{\lambda}}=-1=-\hat{v}^{\prime}(0) .
\end{aligned}
$$

\subsection{The case $q<p, q \neq \frac{p+1}{2}$}

For $q<p$ there are no solutions $\varphi$ which converge to $P_{2}$ for $t \rightarrow-\infty$. In fact, if $\frac{q}{p}<1$, then $P_{2} \in \overline{S_{-}(t)}$ for all $t$ (see Section 2.6). Hence $\dot{u}(t)<0$ for sufficiently small $t$, which implies $u(t) \nrightarrow 0$. On the other hand, there are always $\varphi$ converging to $P_{4}$. It is easy to see this in the case $\frac{p+1}{2}<q$. In fact, $q<p$ implies $P_{4} \in \bar{G}_{-}$and $\frac{p+1}{2}<q$ implies $P_{3}^{-} \notin \bar{G}_{-}$, so that all solutions starting in $G_{-}$at some $t_{0}<T^{*}$ stay in $G_{-}$by Lemma 2.3 and necessarily converge to $P_{4}$ by Thieme's theorem. For $a \neq 0$ (2.4.1) does not have 0 as a solution.

The picture is not so clear for $q<\frac{p+1}{2}$, when $\operatorname{Re} \lambda_{i}<0, i=1,2$. Let us convert $\left(\mathrm{MS}_{p, q}\right)$ into an autonomous system by adding the additional variable $W(t):=e^{2 t}$. By $(2.2 .6)$,

$$
q(t)=q-(q-1) W(t)+(q-1) \frac{W^{2}(t)}{1+W(t)},
$$

so that $t \mapsto(u(t), v(t), W(t))$ satisfies the 3 -dimensional system

$$
\left.\begin{array}{l}
\dot{U}=U(q-U-p V)-(q-1) U W+(q-1) \frac{U W^{2}}{1+W}, \\
\dot{V}=V(-1+U+V), \\
\dot{W}=2 W .
\end{array}\right\} \quad\left(\overline{\mathrm{MS}}_{p}, \lambda\right)
$$

Its linearization at the stationary point $\bar{P}_{4}:=\left(u_{4}, v_{4}, 0\right)$ is, with $\bar{U}:=U-$ $u_{4}, \bar{V}:=V-v_{4}$,

$$
\left(\begin{array}{c}
\bar{U} \\
\bar{V} \\
W
\end{array}\right)=\bar{A}\left(\begin{array}{c}
\bar{U} \\
\bar{V} \\
W
\end{array}\right)+\left(\begin{array}{c}
-\bar{U}^{2}-p \bar{U} \bar{V}-(q-1) \bar{U} W \\
\bar{U} \bar{V}-\bar{V}^{2} \\
0
\end{array}\right)+\left(\begin{array}{c}
(q-1)\left(\bar{U}+u_{4} \frac{W^{2}}{1+W}\right) \\
0 \\
0
\end{array}\right)
$$


with $\bar{A}=\left(\begin{array}{c|c}A & -(q-1) u_{4} \\ \hline 00 & 2\end{array}\right)$.

Because $\operatorname{Re} \lambda_{i}<0, i=1,2$ and $\lambda_{3}=2$ (see Section 2.4), $\bar{P}_{4}$ is a hyperbolic point for $\left(\overline{\mathrm{MS}}_{p, \lambda}\right)$. In the case $\frac{p+1}{2}<q, \bar{P}_{4}$ has a 3 -dimensional stable manifold for $t \rightarrow-\infty$. If $q<\frac{p+1}{2}$, the stable manifold for $t \rightarrow-\infty$ has dimension one, whose tangent vector is given by an eigenvector to $\lambda_{3}$, which is

$$
\begin{aligned}
& \xi_{3}=\left(-(q-1) \frac{\left(2-v_{4}\right)\left(1-v_{4}\right)}{\left(3-v_{4}\right)\left(2-v_{4}\right)+p\left(1-v_{4}\right) v_{4}}\right. \\
&\left.-(q-1) \frac{\left(1-v_{4}\right) v_{4}}{\left(3-v_{4}\right)\left(2-v_{4}\right)+p\left(1-v_{4}\right) v_{4}}, 1\right) .
\end{aligned}
$$

Hence there exists a solution $\psi=(U, V, W)$ converging to $\bar{P}_{4}$ and for which $W\left(t_{0}\right)>0$ for some $t_{0} \in \mathbb{R}$. It follows that $W(t)=W\left(t_{0}\right) e^{2\left(t-t_{0}\right)}$, and there exists $t_{1}$ with $W\left(t_{1}\right)=1$, so that $W\left(t_{0}\right) \cdot e^{2\left(t_{1}-t_{0}\right)}=1$. Then $t \mapsto\left(U\left(t+t_{1}\right), V\left(t+t_{1}\right), W\left(t+t_{1}\right)=e^{2 t}\right)$ is also a solution of $\left(\overline{\mathrm{MS}}_{p, \lambda}\right)$ converging to $\bar{P}_{4}$, and $\varphi(t):=\left(U\left(t+t_{1}\right), V\left(t+t_{1}\right)\right)$ is a solution of $\left(\mathrm{MS}_{p, \lambda}\right)$ converging to $P_{4}$.

The following theorem shows that $P_{4}$ takes the role of $P_{2}$ for $q<p$, $q \neq \frac{p+1}{2}$.

Theorem 5.8. Let $q<p, q \neq \frac{p+1}{2}$. The following conditions are equivalent:

a) $\phi$ is an $M$-solution.

b) We have

$$
\begin{aligned}
\phi(r) & =c_{p, \lambda} r^{-\frac{\lambda}{p-1}}(1+o(1)), \\
\phi^{\prime}(r) & =-c_{p, \lambda} \frac{\lambda}{p-1} r^{-\frac{\lambda}{p-1}-1}(1+o(1)), \quad c_{p, \lambda}:=\left(\frac{(p-\lambda-1) \lambda}{(p-1)^{2}}\right)^{\frac{1}{p-1}} .
\end{aligned}
$$

c) We have

$$
\begin{aligned}
u(t) & =\frac{p-\lambda-1}{p-1}(1+o(1)), \\
v(t) & =\frac{\lambda}{p-1}(1+o(1)) \quad(t \rightarrow-\infty) .
\end{aligned}
$$

d) $\varphi(t) \rightarrow P_{4}$.

Proof. a) $\rightarrow$ d): Similarly as in a) $\rightarrow$ d) of the proof of Theorem 5.7.

d) $\leftrightarrow$ c) and b) $\rightarrow$ a) are trivial. 
c) $\rightarrow$ b): c) implies with (2.2.2)

$$
\begin{aligned}
\phi(r) & =\left(\left(1+r^{2}\right)^{\lambda / 2} r^{-\lambda} \frac{p-q}{p-1}(1+o(1)) \frac{q-1}{p-1}(1+o(1))\right)^{\frac{1}{p-1}} \\
& =c_{p, \lambda} r^{-\frac{\lambda}{p-1}}(1+o(1)), \\
\phi^{\prime}(r) & =-\frac{1}{r} \frac{q-1}{p-1}(1+o(1)) \cdot \phi(r),
\end{aligned}
$$

which is b).

$$
\text { 5.4. The case } q=\frac{p+1}{2}
$$

We will use the functional

$$
H(u, v)=(q-u-q v) u^{\frac{1}{\lambda}} v^{1+\frac{1}{\lambda}}=-G(u, v) u^{\frac{1}{\lambda}} v^{1+\frac{1}{\lambda}}
$$

on $\mathbb{R}_{0}^{+} \times \mathbb{R}_{0}^{+}$. We have $H>0$ on $G_{-}$(the open triangle $\left.P_{1} P_{2} P_{3}^{-}\right), H=0$ on the boundary $\partial G_{-}$of $G_{-}, H<0$ on $G_{+}$, and the maximum value is

$$
H\left(P_{4}\right)=H\left(\frac{1}{2}, \frac{1}{2}\right)=\frac{\lambda}{2^{2+2 / \lambda}}=: c_{\lambda} .
$$

$H$ is constant along the solutions of $\left(\mathrm{EFS}_{p, \frac{p+1}{2}}\right)[4$, p. 510 , case $n=3 m+5]$ in $G_{-}$. These are periodic orbits around $P_{4}$ whose graph is $H^{-1}(c), c \in\left(0, c_{\lambda}\right)$. $H^{-1}(0)$ is the union of the stationary points $P_{1}, P_{2}, P_{3}^{-}$cyclically chained by orbits of $\left(\mathrm{EFS}_{p, \frac{p+1}{2}}\right)[4$, p. 509].

To investigate $\left(M S_{p, \frac{p+1}{2}}\right)$, we need the following two lemmas.

Lemma 5.9. Let $\varphi$ be a solution of $\left(M S_{p, \frac{p+1}{2}}\right)$ on $\left(T_{0}, T\right)$. Then

$$
\frac{d}{d t} H(\varphi(t))=q \cdot u^{\frac{1}{\lambda}} v^{1+\frac{1}{\lambda}} \frac{e^{2 t}}{1+e^{2 t}} W(u, v), \quad u=u(t), v=v(t) .
$$

Consequently, if $B$ is a bounded subset of $\mathbb{R}^{+} \times \mathbb{R}^{+}$,

$$
M_{B}:=\sup _{(u, v) \in B} q u^{\frac{1}{\lambda}} v^{1+\frac{1}{\lambda}}|W(u, v)|
$$

and $\varphi(t) \in B$ on $\left[t_{1}, t_{2}\right] \subset\left(T_{0}, T\right)$ then

$$
\left|H\left(\varphi\left(t_{2}\right)\right)-H\left(\varphi\left(t_{1}\right)\right)\right| \leq \frac{1}{2} M_{B}\left(e^{2 t_{2}}-e^{2 t_{1}}\right) .
$$


Proof. We have, with $q(t)=q-g(t), g(t)=\lambda \frac{e^{2 t}}{1+e^{2 t}}$,

$$
\begin{aligned}
& \frac{d}{d t} H(\varphi(t))=(q-u-q v)^{\cdot} u^{\frac{1}{\lambda}} v^{1+\frac{1}{\lambda}}+(q-u-q v) \frac{1}{\lambda} u^{\frac{1}{\lambda}-1} \dot{u} v^{1+\frac{1}{\lambda}} \\
& +(q-u-q v) u^{\frac{1}{\lambda}}\left(1+\frac{1}{\lambda}\right) v^{\frac{1}{\lambda}} \cdot \dot{v} \\
& =u^{\frac{1}{\lambda}} v^{1+\frac{1}{\lambda}}(-u(q(t)-u-p v)-q v(-1+u+v) \\
& +\frac{1}{\lambda}(q-u-q v)(q(t)-u-p v) \\
& \left.+(q-u-q v)\left(1+\frac{1}{\lambda}\right)(-1+u+v)\right) \\
& =u^{\frac{1}{\lambda}} v^{1+\frac{1}{\lambda}}\left((q(t)-u-p v)\left(-u+\frac{1}{\lambda}(q-u-q v)\right)\right. \\
& \left.+(-1+u+v)\left(-q v+\left(1+\frac{1}{\lambda}\right)(q-u-q v)\right)\right) \\
& =u^{\frac{1}{\lambda}} v^{1+\frac{1}{\lambda}}\left((q(t)-u-p v)\left(-\left(1+\frac{1}{\lambda}\right) u+\frac{q}{\lambda}-\frac{q}{\lambda} v\right)\right. \\
& +(-1+u+v)\left(\left(1+\frac{1}{\lambda}\right) q-\left(1+\frac{1}{\lambda}\right) u\right. \\
& \left.\left.-\left(q+\frac{q^{2}}{\lambda}\right) v\right)\right) \\
& =u^{\frac{1}{\lambda}} v^{1+\frac{1}{\lambda}}\left((q-g(t)-u-p v) \frac{q}{\lambda}(1-u-v)\right. \\
& \left.+(-1+u+v)\left(\frac{q^{2}}{\lambda}-\frac{q}{\lambda} u-\left(1+\frac{q}{\lambda}\right) v\right)\right) \\
& =u^{\frac{1}{\lambda}} v^{1+\frac{1}{\lambda}}\left(\frac{q}{\lambda} g(t)(-1+u+v)\right. \\
& +(-1+u+v) \cdot 0) \text { (because } p-\lambda-q=0), \\
& =\frac{q}{\lambda} u^{\frac{1}{\lambda}} v^{1+\frac{1}{\lambda}} g(t) W(u, v)=q \cdot u^{\frac{1}{\lambda}} v^{1+\frac{1}{\lambda}} \frac{e^{2 t}}{1+e^{2 t}} W(u, v) .
\end{aligned}
$$

The last assertion follows immediately.

In the sequel we will apply the name $F$-, $E$-, $M$-solution also to the corresponding $\varphi$.

Lemma 5.10. Let $\varphi$ be a solution of $\left(M S_{p, \frac{p+1}{2}}\right)$ on $\left(T_{0}, T\right)$. Then $\varphi$ is an $F$-solution iff there exists $t_{0}$ with $\varphi\left(t_{0}\right) \in G_{+} \cup G_{0}$. In this case $\varphi(t) \in G_{+}$ for $t<t_{0}$. 
Proof. The necessity of the condition follows from Theorem 3.1.d). Conversely, if $\varphi\left(t_{0}\right) \in G_{+}$, then $\varphi(t) \in G_{+}$for $t \leq t_{0}$ by Lemma 2.3. Because $G_{+} \subset W_{+}$, we have $\frac{d}{d t} H(\varphi(t))>0$ by (5.4.1), which implies

$$
H(\varphi(t))<H\left(\varphi\left(t_{0}\right)\right)=: c_{0} \text { for } t<t_{0} .
$$

If $\varphi$ were not an $F$-solution, by Theorem $3.1 L^{-}(\varphi)$ would be a bounded set lying outside $H^{-1}\left(\left(c_{0}, c_{\lambda}\right]\right)$, which is an open neighborhood of $G_{-} \cup G_{0}$, because $c_{0}<0$. But this contradicts Thieme's theorem. Hence $\varphi$ is an $F$ solution. If $\varphi\left(t_{0}\right) \in G_{0}$, then $\varphi(t) \in G_{+}$for $t<t_{0}$ by Lemma 2.3 and the conclusion follows again.

Lemma 5.10 implies that the $F$-solutions, which are all in $G_{+}$for small $t$ by Theorem 3.1 either stay in $G_{+}$or (see Lemma 9.5) enter $G_{-}$and stay in $G_{-}$for larger $t$ by Lemma 2.3. It also implies that the $E$ - and $M$-solutions are exactly those which totally lie in $G_{-}$. For the $E$-solutions $\varphi$, we always have $H(\varphi(-\infty)):=\lim _{t \rightarrow-\infty} H(\varphi(t))=0$ by Theorem 4.1 .

The $M$-solutions are the object of the following theorem. For $c \in\left(0, c_{\lambda}\right)$, let $\Gamma_{c}=H^{-1}(c)$; for a set $K \in \mathbb{R}^{2}, r>0$ let $B_{r}(K):=\cup_{z \in K} B_{r}(z)$ (the open ball about $z$ with radius $r$ ), and for any $z \in \mathbb{R}^{2} \backslash\left\{P_{4}\right\}$ let $R(z)$ be the ray $\left\{P_{4}+s\left(z-P_{4}\right), s \geq 0\right\}$. And let $\Omega_{c}:=\{(u, v): H(u, v)>c\}$, the region inside $\Gamma_{c}$.

Theorem 5.11. Let $q=\frac{p+1}{2}$.

a) If $\varphi$ is an $M$-solution, then $\varphi$ lies in $G_{-}$and $H(\varphi(-\infty)) \in\left[0, c_{\lambda}\right]$ exists. Conversely we have:

b) $\forall c \in\left(0, c_{\lambda}\right), \forall \varepsilon<\frac{1}{2} \min \left(\left(c, c_{\lambda}-c\right) \exists \tau \in \mathbb{R} \quad \forall t_{0} \leq \tau, \forall z \in \Gamma_{c} \exists z_{0} \in\right.$ $B_{\varepsilon}(z) \cap R(z)$ :

$$
H\left(\varphi\left(-\infty, t_{0}, z_{0}\right)\right)=c .
$$

c) For $c=c_{\lambda}$ there exists a unique $M$-solution $\varphi$ such that $H(\varphi(-\infty))=$ $c_{\lambda}$.

Proof. a) follows from the preceding observations and (5.4.2). As for b), for $(u, v) \in G_{-}$, let us consider the solution $\varphi\left(\cdot, t_{0}\right)$ of the initial value problem $\varphi\left(t_{0}\right)=(u, v)$ with $t_{0} \in \mathbb{R}$. Obviously,

$$
\mathbb{R}=A \cup B \cup C
$$

is a partition of $\mathbb{R}$ into the subsets

$$
\begin{aligned}
& A:=\left\{t_{0} \in \mathbb{R} ; \varphi\left(\cdot, t_{0}\right) \text { is an } M \text {-solution with } H\left(\varphi\left(-\infty, t_{0}\right)\right)>0\right\} \\
& B:=\left\{t_{0} \in \mathbb{R} ; \varphi\left(\cdot, t_{0}\right) \text { is an } F \text {-solution }\right\} \\
& C:=\left\{t_{0} \in \mathbb{R} ; \varphi\left(\cdot, t_{0}\right) \text { is a solution in } G_{-} \text {with } H\left(\varphi\left(-\infty, t_{0}\right)\right)=0\right\}
\end{aligned}
$$


The set $A$ contains the interval $\left(-\infty, s_{0}\right)$, where $s_{0}$ is defined by $M_{G_{-}} e^{2 s_{0}}=$ $H(u, v)$. In fact, if $t_{0}<s_{0}$, then $M_{G_{-}} e^{2 t_{0}}<H(u, v)$, and, as long as $\varphi(s) \in G_{-}$on $\left(t, t_{0}\right]$, we have with (5.4.2)

$$
H(u, v)-H\left(\varphi\left(t, t_{0}\right)\right) \leq \frac{1}{2} M_{G_{-}}\left(e^{2 t_{0}}-e^{2 t}\right)<\frac{1}{2} H(u, v),
$$

which implies

$$
H\left(\varphi\left(t, t_{0}\right)\right)>\frac{1}{2} H(u, v)>0, \varphi\left(t, t_{0}\right) \in G_{-} \text {for all } t \leq t_{0},
$$

so that $\left(-\infty, s_{0}\right) \subset A$ and $\left(-\infty, s_{0}\right) \cap B=\left(-\infty, s_{0}\right) \cap C=\varnothing$. Now we vary $(u, v) \in G_{-}$and consider $s_{0}=s_{0}(u, v):=\frac{1}{2} \ln \frac{H(u, v)}{M_{G_{-}}}$and $A=A(u, v)$. Let $U$ be an open set in $G_{-}$with closure $\bar{U} \subset G_{-}$and define

$$
\sigma_{U}:=\min _{(u, v) \in \bar{U}} s_{0}(u, v)>-\infty .
$$

Given $\varepsilon>0$, it follows from (5.4.2) with $t_{1}=-\infty$ and $t_{2}:=t$ that

$$
\begin{aligned}
& \exists s_{\varepsilon}<\sigma_{U}, \forall t \leq s_{\varepsilon}, \forall(u, v) \in U, \forall t_{0} \in A(u, v): \\
& \left|H\left(\varphi\left(-\infty, t_{0}, u, v\right)\right)-H\left(\varphi\left(t, t_{0}, u, v\right)\right)\right|<\varepsilon .
\end{aligned}
$$

Now we show that i) the set

$$
\Omega:=\left\{\left(t_{0}, u, v\right) ;(u, v) \in G_{-}, t_{0} \in A(u, v)\right\}
$$

is open in $\mathbb{R} \times \mathbb{R}_{+}^{2}$ and ii) the mapping

$$
\mathcal{H}: \Omega \rightarrow\left(0, c_{\lambda}\right], \quad\left(t_{0}, u, v\right) \longmapsto H\left(\varphi\left(-\infty, t_{0}, u, v\right)\right)
$$

is continuous. For i) let $\left(t_{1}, u_{1}, v_{1}\right) \in \Omega$. Because $t_{1}<A\left(u_{1}, v_{1}\right)$, the set $\left\{\varphi\left(t, t_{1}, u_{1}, v_{1}\right), t \leq t_{1}\right\}$ has a positive distance from $H^{-1}(0)$ and there exists an open set $U$ with closure $\bar{U} \subset G_{-}$such that $\left\{\varphi\left(t, t_{1}, u, v\right), t \leq t_{1}\right\} \subset$ $U$. Now let $\varepsilon>0, s_{\varepsilon}<\sigma_{U}$ be chosen as above, $\delta:=\sigma_{U}-s_{\varepsilon}>0$, and $s_{\varepsilon}^{*}:=\min \left(s_{\varepsilon}-\delta, t_{1}-\delta\right)$. Then for all $(u, v) \in U$ we have $\sigma_{\varepsilon}^{*} \in A(u, v)$ (because $\left.s_{\varepsilon}^{*}<s_{\varepsilon}<\sigma_{U} \leq \sigma_{0}(u, v)\right)$ and for all $t \leq s_{\varepsilon}^{*}\left(<s_{\varepsilon}\right)$ we have with (5.4.3)

$$
\left|H\left(\varphi\left(-\infty, \sigma_{\varepsilon}^{*}, u, v\right)\right)-H\left(\varphi\left(t, \sigma_{\varepsilon}^{*}, u, v\right)\right)\right|<\varepsilon .
$$

The continuous dependence of the initial conditions implies the existence of a neighborhood of $\left(t_{1}, u_{1}, v_{1}\right)$ which has the form $\left(t_{1}-s_{1}, t_{1}+\delta_{1}\right) \times V, \delta_{1}<$ $\delta, \bar{V} \subset U$, such that for all $t_{2} \in\left(t_{1}-\delta_{1}, t_{1}+\delta_{1}\right)$ and $(u, v) \in V$ we have $s_{\varepsilon}^{*}<t_{2}$ and

$$
\varphi\left(s_{\varepsilon}^{*}, t_{2}, u, v\right) \in U .
$$

This implies $s_{\varepsilon}^{*} \in A\left(\varphi\left(s_{\varepsilon}^{*}, t_{2}, u, v\right)\right)$ as above and $\varphi\left(\cdot, t_{2}, u, v\right)$ $=\varphi\left(\cdot, \sigma_{\varepsilon}^{*}, \varphi\left(s_{\varepsilon}^{*}, t_{2}, u, v\right)\right)$ is an $M$-solution with $H$-limit $>0$, that is, $t_{2} \in$ $A(u, v)$. This proves i). Furthermore, for the same $\left(t_{2}, u, v\right)$ we have by (5.4.4),

$$
\left|H\left(\varphi\left(-\infty, t_{2}, u, v\right)\right)-H\left(\varphi\left(s_{\varepsilon}^{*}, t_{2}, u, v\right)\right)\right|<\varepsilon,
$$


in particular,

$$
\left|H\left(\varphi\left(-\infty, t_{1}, u_{1}, v_{1}\right)\right)-H\left(\varphi\left(s_{\varepsilon}^{*}, t_{1}, u_{1}, v_{1}\right)\right)\right|<\varepsilon,
$$

and $\delta_{1}$ and $V$ can be assumed to be small enough for having

$$
\left|H\left(\varphi\left(s_{\varepsilon}^{*}, t_{2}, u, v\right)\right)-H\left(\varphi\left(s_{\varepsilon}^{*}, t_{1}, u_{1}, v_{1}\right)\right)\right|<\varepsilon .
$$

This shows ii) also.

To prove b), let $c$ and $\varepsilon$ be given. Let $B=\bar{\Omega}_{c-\frac{1}{2} \min \left(\left(c, c_{\lambda}-c\right)\right.}$. It is easy to see that there exists $\tau \leq \sigma_{B}$ and $\delta<\frac{\varepsilon}{4}$ such that for all $\forall t_{0} \leq \tau, t \leq$ $t_{0}, z, z^{\prime} \in B$ with $\left|z-z^{\prime}\right|<\delta$ we have

$$
\left|H\left(\varphi\left(t, t_{0}, z_{0}\right)\right)-H\left(z^{\prime}\right)\right|<\frac{\varepsilon}{4}
$$

(one uses Lemma 5.9 and the continuity of $\mathcal{H}$ ).This implies:

$$
\forall z \in B_{\delta}\left(\Gamma_{d_{i}}\right) \forall t \leq t_{0}: \varphi\left(t, t_{0}, z_{0}\right) \in B_{\frac{\varepsilon}{4}}\left(\Gamma_{d_{i}}\right) i=1,2
$$

where $d_{1}:=c-\frac{\varepsilon}{2}$ and $d_{2}:=c+\frac{\varepsilon}{2}$, in particular we have

$$
\begin{aligned}
& \forall z \in B_{\delta}\left(\Gamma_{d_{1}}\right): \mathcal{H}\left(t_{0}, z\right) \leq d_{1}+\frac{\varepsilon}{4}<c, \\
& \forall z \in B_{\delta}\left(\Gamma_{d_{2}}\right): \mathcal{H}\left(t_{0}, z\right) \geq d_{2}-\frac{\varepsilon}{4}>c .
\end{aligned}
$$

Now, if $z \in \Gamma_{c}$ and $\left\{z_{i}\right\}:=\Gamma_{d_{i}} \cap R(z), i=1,2$, then $\mathcal{H}\left(t_{0}, z_{1}\right)<c<$ $\mathcal{H}\left(t_{0}, z_{2}\right)$, and the continuity of $\mathcal{H}$ implies the existence of some $z_{0} \in R(z)$ between $z_{1}$ and $z_{2}$ (so that $z_{0} \in B_{\varepsilon}(z)$ ) with $\mathcal{H}\left(t_{0}, z_{0}\right)=c$.

To prove c), we first derive an a-priori estimate for such a $\varphi$. We have $\varphi(t) \in G_{-}$for all $t$, and with (5.4.2)

$$
\left|H(u(t), v(t))-H\left(P_{4}\right)\right| \leq \frac{1}{2} M_{G_{-}} e^{2 t} .
$$

By Taylor's formula, for all $(u, v)$ in a neighborhood of $P_{4}$ one has

$$
\begin{aligned}
H(u, v)-H\left(P_{4}\right) & =\frac{p+1}{(p-1) 2^{\frac{p+2}{p-1}}}\left(u-\frac{1}{2}\right)^{2}+2\left(u-\frac{1}{2}\right)\left(v-\frac{1}{2}\right) \\
& +p\left(v-\frac{1}{2}\right)^{2}+o\left(\left|\left(u-\frac{1}{2}, v-\frac{1}{2}\right)\right|^{2}\right),
\end{aligned}
$$

which implies

$$
\left|u-\frac{1}{2}\right|^{2}+\left|v-\frac{1}{2}\right|^{2} \leq C_{1}\left|H(u, v)-H\left(P_{4}\right)\right|
$$

for some $C_{1}>0$ in a neighborhood of $\left(\frac{1}{2}, \frac{1}{2}\right)$. The decomposition (2.4.1) for $P_{4}$ is

$$
\dot{\psi}(t)=A \psi(t)+g_{1}(\psi(t))+g_{2}(t, \psi(t))
$$


with

$$
\begin{aligned}
& A=\left(\begin{array}{rr}
-\frac{1}{2} & -\frac{p}{2} \\
\frac{1}{2} & \frac{1}{2}
\end{array}\right), \quad \lambda_{1,2}= \pm \frac{1}{2} \sqrt{p-1} \cdot i, \\
& g_{1}(z):=\left(\begin{array}{c}
-\bar{u}^{2}-p \bar{u} \bar{v} \\
\bar{u} \bar{v}+\bar{v}^{2}
\end{array}\right), g_{2}(t, z):=\left(\begin{array}{c}
-(q-1)\left(\bar{u}+\frac{1}{2}\right) \frac{e^{2 t}}{1+e^{2 t}} \\
0
\end{array}\right) \text {, }
\end{aligned}
$$

(see Section 2.4). Hence for small $t, \sigma \in \mathbb{R}$,

$$
\psi(t)=e^{(t-\sigma) A} \psi(\sigma)+\int_{\sigma}^{t} e^{(t-s) A}\left(g_{1}(\psi(s))+g_{2}(s, \psi(s))\right) d s .
$$

We want to let $\sigma \rightarrow-\infty$. To this end, we note that it follows from (5.4.5) and (5.4.6) that

$$
|\psi(t)|=O\left(e^{t}\right), t \rightarrow-\infty .
$$

Furthermore, $s \longmapsto e^{s A}$ is bounded on $\mathbb{R}$, and (5.4.7) and (5.4.8) imply for $\sigma \rightarrow-\infty$

$$
\psi(t)=\int_{-\infty}^{t} e^{(t-\sigma) A}\left(g_{1}(\psi(s))+g_{2}(s, \psi(s))\right) d s .
$$

Now we are going to construct a $\varphi(t) \rightarrow P_{4}$, that is, a solution $\psi(t) \rightarrow 0$ of (5.4.9). The estimate (5.4.8) suggests to consider the Banach space

$$
X_{S}:=\left\{\psi \in C(-\infty, S], t \longmapsto e^{-t}|\psi(t)| \text { is bounded on }(-\infty, S]\right\}
$$

equipped with the norm

$$
\|\psi\|:=\sup _{t \leq S} e^{-t}|\psi(t)|,|z|:=\left|z_{1}\right|+\left|z_{2}\right|, z=\left(z_{1}, z_{2}\right) \in \mathbb{R}^{2} .
$$

If we denote by $F \psi(t)$ the right hand side of (5.4.9), the definitions of $g_{1}, g_{2}$ and (5.4.8) give with constants $C_{2}, C_{3}, C_{4}$,

$$
\begin{aligned}
\|F \psi\| & \leq \sup _{t \in S} e^{-t} \int_{-\infty}^{t} C_{2}\left(|\psi(s)|^{2}+|\psi(s)|+1\right) e^{2 s} d s \\
& \leq C_{2} \sup _{t \in S} e^{-t} \int_{-\infty}^{t}\left(\left(\|\psi\|^{2}+1\right) e^{2 s}+\|\psi\| e^{3 s}\right) d s \\
& \leq C_{2}\left(\left(\|\psi\|^{2}+1\right) e^{S}+\|\psi\| e^{2 S}\right), \\
\left\|F \psi_{1}-F \psi_{2}\right\| & \leq \sup _{t \in S} e^{-t} \int_{-\infty}^{t} C_{3}\left(\left|\bar{u}_{1}^{2}-u_{2}^{-2}\right|\right. \\
& \left.+\left|\bar{u}_{1} \bar{v}_{1}-\bar{u}_{2} \bar{v}_{2}\right|+\left|\bar{v}_{1}^{2}-\bar{v}_{2}^{2}\right|+\left|\bar{u}_{1}-\bar{u}_{2}\right| e^{2 s}\right) d s \\
& \leq C_{4} \sup _{t \in S} e^{-t} \int_{-\infty}^{t}\left(\left\|\psi_{1}\right\|+\left\|\psi_{2}\right\|+e^{S}\right)\left\|\psi_{1}-\psi_{2}\right\| e^{2 s} d s \\
& \leq C_{4}\left(\left\|\psi_{1}\right\|+\left\|\psi_{2}\right\|+e^{S}\right) e^{S}\left\|\psi_{1}-\psi_{2}\right\| .
\end{aligned}
$$


For any $r>0, S \in \mathbb{R}$ which satisfy

$$
C_{2}\left(r^{2}+1+r e^{S}\right) e^{S} \leq r, C_{4}\left(2 r+e^{S}\right) e^{S}<1
$$

$F$ is a contraction in the closed ball in $X_{S}$ of radius $r$ about the origin. The unique fixed point $\psi$ is a solution of (5.4.9), hence $\varphi:=\psi+\left(\frac{1}{2}, \frac{1}{2}\right)$ solves $\left(M S_{p, \frac{p+1}{2}}\right)$ and $\psi(t) \rightarrow P_{4}$. Now let $\varphi_{1}, \varphi_{2}$ be two solutions with $\varphi_{1}(t) \varphi_{2}(t) \rightarrow P_{4}$. Then $\psi_{i}:=\varphi_{i}-\left(\frac{1}{2}, \frac{1}{2}\right)$ satisfies (5.4.9) and there exist $r_{i}>0, S_{i} \in \mathbb{R}$ with $\left\|\psi_{i}\right\| \leq r_{i}$ in $X_{S_{i}}, i=1,2$. There exists $S \leq \min \left(S_{1}, S_{2}\right)$ which satisfies (5.4.10) for $r:=\max \left(r_{1}, r_{2}\right)$ and $\left\|\psi_{i}\right\| \leq r$ in $X_{S}$. Hence $\psi_{1}=\psi_{2}$ and $\varphi_{1}=\varphi_{2}$.

Remark 3. We do not know if there exists an $M$-solution $\varphi$ with $L^{-}(\varphi)=$ $\partial G_{-}$(C) of Thieme's theorem) and if for all $(u, v) \in G_{-}$there exists $t_{o}$ such that $\varphi\left(\cdot, t_{0}, u, v\right)$ is an $E$-solution.

\section{Solutions with $R<\infty$}

In this section, we consider solutions $\phi$ on $\left(R_{0}, R\right), 0 \leq R_{0}<R \leq \infty$ and corresponding $\varphi$ on $\left(T_{0}, T\right),-\infty \leq T_{0}<T \leq+\infty, T_{0}=\ln R_{0}, T=\ln R$. The following theorem implies the existence of $\phi$ with $R<\infty$, that is, $\lim _{r \uparrow R} \phi(r)=0$ for finite $R>0$.

Theorem 6.1. The following conditions are equivalent:

a) $R<\infty$.

b) $T<\infty$.

c) $C^{+}(\varphi)$ is unbounded as $t \uparrow T$.

d) $\exists t_{0} \in\left(T_{0}, T\right)$ with $v\left(t_{0}\right)>1$.

e) $v(t) \uparrow \infty$ as $t \uparrow T$.

In this case, $u(t) \downarrow 0$ as $t \uparrow T$, and $L^{+}(\varphi)=\varnothing($ we write $\varphi \rightarrow \varnothing)$.

Proof. a) $\leftrightarrow$ b) follows from the definitions of $R$ and $T$, and e) $\rightarrow$ d) is clear.

b) $\rightarrow$ c): If $C^{+}(\varphi)$ were bounded, the closure of $C^{+}(\varphi)$ would be a compact subset of $\mathbb{R}_{0}^{+} \times \mathbb{R}_{0}^{+}$. But because $T<\infty$, it cannot contain a point of the axes, it is a subset of $\mathbb{R}^{+} \times \mathbb{R}^{+}$. This implies that $\varphi$ can be extended beyond $T$, which is a contradiction.

c) $\rightarrow$ d): If d) were not true, $u$ is unbounded as $t \rightarrow-\infty$. We have

$$
\dot{u} \leq u(q(t)-u)<u(q-u) .
$$

The solution of the initial-value problem $\dot{z}=z(q-z), z\left(t_{0}\right)=u\left(t_{0}\right)=: u_{0}$ for some $t_{0} \in\left(T_{0}, T\right)$ is

$$
z(t)=\frac{q}{1+c e^{-q t}}, \quad c:=\frac{q-u_{0}}{u_{0}} e^{q t_{0}}
$$


on the interval $\left(-\frac{1}{q} \ln \left(-\frac{1}{c}\right), \infty\right)$ for $u_{0}>q$ and on $\mathbb{R}$ for $u_{0} \leq q$, and $u(t) \leq z(t)$ for $t_{0} \leq t<T$. Because $z(t) \rightarrow q, u$ must be bounded, which is a contradiction.

$\mathrm{d}) \rightarrow \mathrm{b})$, e): We have $\dot{v}>v(-1+v)$. The solution $z$ of the initial-value problem $\dot{z}=z(-1+z), z\left(t_{0}\right)=v\left(t_{0}\right)=: v_{0}$ is

$$
z(t)=\frac{1}{1-c e^{t}}, \quad c:=\frac{v_{0}-1}{v_{0}} e^{t_{0}}>0
$$

on the interval $\left(-\infty, \ln \frac{1}{c}\right)$, and $z(t)<v(t)$ for those $t \geq t_{0}$ where $z$ and $v$ exist. This implies $T<\infty$ and necessarily $v(t) \uparrow \infty$ as $t \uparrow T$.

Now we show $u(t) \downarrow 0$ as $t \uparrow T$. Without loss of generality we can assume $v_{0}>\max \left\{1, \frac{q}{p}\right\}$, which implies $\varphi(t) \in W_{+} \cap S_{-}(t)$ on $\left(t_{0}, T\right)$. Hence the function $\hat{v}(u):=v(t(u))$ exists for $\left(L, u_{0}\right), L:=\lim _{t \uparrow T} u(t)$. $\hat{v}$ satisfies the differential equation

$$
\begin{aligned}
\hat{v}^{\prime}(u) & =\frac{\dot{v}(t(u))}{\dot{u}(t(u))}=\frac{v(t(u))(-1+u+v(t(u)))}{u(q(t(u))-u-v(t(u)))} \\
& =\frac{\hat{v}(u)(-1+u+\hat{v}(u))}{u(q(t(u))-u-\hat{v}(u))}, \quad L \leq u \leq u_{0} .
\end{aligned}
$$

We consider the function $y(u):=c\left(\frac{u_{0}}{u}\right)^{\frac{p+1}{p}}$ for some $c>\max \left\{v_{0}, \frac{(q-1) p+q}{p^{2}}\right\}$. Then we have for $L \leq u \leq u_{0}$ :

1.

$$
\begin{gathered}
y(u) \geq y\left(u_{0}\right)=c \geq v_{0}>\max \left\{1, \frac{q}{p}\right\}, \\
\text { hence } \quad q-u-p \cdot y(u)<0 \text { and }-1+u+y(u)>0,
\end{gathered}
$$

2.

$$
\begin{aligned}
p^{2} y(u) & >((q-1) p+q)\left(\frac{u_{0}}{u}\right)^{\frac{p+1}{p}}>(q-1) p+q \\
& \Longrightarrow-q p-q+p u+p y(u)+p^{2} y(u)>-p+p u+p y(u) \\
& \Longrightarrow-(p+1)(q-u-p y(u))>p(-1+u+y(u)) \\
& \Longrightarrow-\frac{p+1}{p}<\frac{-1+u+y(u)}{q-u-p y(u)} \text { with }(6.3) \\
& \Longrightarrow y^{\prime}(u)=-\frac{p+1}{p} \frac{y(u)}{u}<\frac{y(u)(-1+u+y(u))}{u(1-u-y(u))} .
\end{aligned}
$$

It follows from (6.1), (6.4) and (6.2) that

$$
\hat{v}(u)<y(u), \quad u \leq u_{0},
$$

hence $u(t)^{\frac{p+1}{p}}<c u_{0}^{\frac{p+1}{p}} v(t)^{-1} \rightarrow 0(t \uparrow T)$ with c).

Corollary 6.2. The following conditions are equivalent: 
a) $R=\infty$.

b) $T=\infty$.

c) $C^{+}(\varphi)$ is bounded as $t \rightarrow \infty$.

d) $v \leq 1$.

e) $\varphi$ converges to a fixed point of $\left(\operatorname{EFS}_{p, q}\right)$, that is, either $\varphi(t) \rightarrow P_{2}$ or $\varphi(t) \rightarrow P_{3}^{+}$for $t \rightarrow \infty$.

Proof. The equivalence a) $\leftrightarrow \mathrm{b}) \leftrightarrow \mathrm{c}) \leftrightarrow$ d) follows from Theorem 6.1.

c) $\rightarrow$ e) follows from Thieme's theorem and the absence of the situations (B) and (C) at $\left(\mathrm{EFS}_{p, 1}\right)$ (see Section 2.5).

e) $\rightarrow$ c) is obvious.

Definition. The solutions $\phi$ of $\left(\mathrm{M}_{p, \lambda}\right)$ with $R=\infty$ for which $\varphi(t) \rightarrow P_{2}$, will be called $P_{2}$-solutions; those for which $\varphi(t) \rightarrow P_{3}^{+}$, will be called $P_{3}^{+}$solutions. In the literature, these solutions are called solutions of "fast" and "slow" decay, respectively.

We will investigate the existence and the properties of the $P_{2^{-}}$and $P_{3}^{+}$solutions in the next two sections.

\section{7. $P_{2}$-solutions (solutions of fast decay)}

In this section we consider $p, q>1$ and solutions $\phi$ on $\left(R_{0}, \infty\right)$ and the corresponding $\varphi$ on $\left(T_{0}, \infty\right), T_{0}:=\ln R_{0}$.

We convince ourselves that there are solutions $\varphi$ tending to $P_{2}$ as $t \rightarrow$ $+\infty$. As in Section 5.3, we convert ( $\left.\mathrm{MS}_{p, \lambda}\right)$ into an autonomous system by adding the additional variable $W(t):=e^{-2 t}$. By $(2.2 .7)$,

$$
q(t)=1+(q-1) W(t)-(q-1) \frac{W^{2}(t)}{1+W(t)},
$$

so that $t \mapsto(u(t), v(t), W(t))$ satisfies the 3-dimensional system

$$
\left.\begin{array}{rl}
\dot{U} & =U(1-U-p V)+(q-1) U W-(q-1) \frac{U W^{2}}{1+W}, \\
\dot{V} & =V(-1+U+V), \\
\dot{W} & =-2 W .
\end{array}\right\}
$$

Its linearization at the stationary point $\bar{P}_{2}:=(1,0,0)$ with $\bar{V}=V-1$, is

$$
\left(\begin{array}{c}
U \\
\bar{V} \\
W
\end{array}\right)=\bar{A}\left(\begin{array}{c}
U \\
\bar{V} \\
W
\end{array}\right)+\left(\begin{array}{c}
-U^{2}-p U \bar{V}+(q-1) U W-(q-1) \frac{W^{2}}{1+W} \\
U \bar{V}+\bar{V}^{2} \\
0
\end{array}\right)
$$

with $\bar{A}=\left(\begin{array}{ccc}-(p-1) & 0 & 0 \\ 1 & 1 & 0 \\ 0 & 0 & -2\end{array}\right)$. The eigenvalues are $\lambda_{1}=-(p-1), \lambda_{2}=1$, $\lambda_{3}=-2$, and the corresponding eigenvectors $\bar{\xi}_{1}=(p,-1,0), \bar{\xi}_{2}=(0,1,0)$, $\bar{\xi}_{3}=(0,0,1)$. 
The plane through $\bar{\xi}_{1}$ and $\bar{\xi}_{3}$ is tangential to the two-dimensional stable manifold in $\bar{P}_{2}$. Consequently, there is a solution $\psi=(U, V, W)$ of $\left(\overline{\mathrm{MP}_{p, \lambda}}\right)$ tending to $\bar{P}_{2}$ for $t \rightarrow+\infty$ such that there exists $t_{0} \in \mathbb{R}$ with $\psi(t) \in$ $\mathbb{R}^{+} \times \mathbb{R}^{+} \times \mathbb{R}^{+}$for $t \geq t_{0}$. We have $W(t)=W\left(t_{0}\right) e^{-2\left(t-t_{0}\right)}$. We define $t_{1}$ by $W\left(t_{0}\right) e^{-2\left(t_{1}-t_{0}\right)}=1$. Then $t \mapsto \psi\left(t+t_{1}\right)$ is also a solution in $\mathbb{R}^{+} \times$ $\mathbb{R}^{+} \times \mathbb{R}^{+}$for $t \geq t_{0}-t_{1}$ and $W\left(t+t_{1}\right)=W\left(t_{0}\right) e^{-2\left(t+t_{1}-t_{0}\right)}=e^{-2 t}$, so that $\varphi(t):=\left(U\left(t+t_{1}\right), V\left(t+t_{1}\right)\right) \in \mathbb{R}^{+} \times \mathbb{R}^{+}$for $t \geq t_{0}-t_{1}$ solves $\left(\mathrm{MS}_{p, \lambda}\right)$ and converges to $P_{2}$ as $t \rightarrow \infty$.

We first state the following lemma.

Lemma 7.1. Let $\alpha, \gamma>0 ; K, L, M \geq 0$ with $\beta:=\frac{L}{\alpha}+\frac{M}{\gamma}<1$. Let $u$ : $[\tau, \infty) \rightarrow[0, \infty)$ be continuous and bounded, and assume for $t \geq \tau$

$$
u(t) \leq K e^{-\alpha t}+L \int_{\tau}^{t} e^{-\alpha(t-s)} u(s) d s+M \int_{\tau}^{\infty} e^{-\gamma s} u(t+s) d s .
$$

Then for $t \geq \tau$

$$
u(t) \leq K^{*} e^{-\left(\frac{L}{1-\beta}-\alpha\right) t}, K^{*}:=\frac{K}{1-\beta} e^{-\frac{L}{1-\beta} \tau} .
$$

Sketch of proof: Define $v(t):=\sup _{s \geq t} u(s)$ and $z(t):=e^{\alpha t} v(t)$. Then

$$
z(t) \leq \frac{K}{1-\beta}+\frac{L}{1-\beta} \int_{\tau}^{t} z(s) d s
$$

and Gronwall's inequality proves the assertion.

The following theorem characterizes the $P_{2}$-solutions.

Theorem 7.2. The following conditions are equivalent:

a) $\phi$ is a $P_{2}$-solution, that is, $\varphi(t) \rightarrow P_{2}(t \rightarrow \infty)$.

b) There exists $c>0$ such that

$$
\phi(r)=\frac{c}{r}(1+o(1)), \quad \phi^{\prime}(r)=-\frac{c}{r^{2}}(1+o(1)) \quad(r \rightarrow \infty) .
$$

c) There exist $c_{1}>0$ and $c_{2} \in \mathbb{R}$ such that

$$
\begin{aligned}
u(t) & =e^{-(p-1) t} c_{1}(1+o(1)), \\
v(t)-1 & =e^{-(p-1) t} c_{2}(1+o(1)) \quad(t \rightarrow \infty) .
\end{aligned}
$$

In this case, $c_{1}=c^{p-1}, c_{2}=-\frac{1}{p} c^{p-1}$, and $\lim _{u \rightarrow 0} \hat{v}^{\prime}(u)=\hat{v}^{\prime}(0)=-\frac{1}{p}$.

Proof. a) $\rightarrow$ b), c): The linearization at $P_{2}$ is (see Section 2.5 and $(2.2 .7)$ )

$$
\left(\begin{array}{l}
u \\
\bar{v}
\end{array}\right)=A\left(\begin{array}{c}
u \\
\bar{v}
\end{array}\right)+\left(\begin{array}{c}
-u^{2}-p u \bar{v} \\
u \bar{v}+\bar{v}^{2}
\end{array}\right)+\left(\begin{array}{c}
(q-1) u \frac{e^{-2 t}}{1+e^{-2 t}} \\
0
\end{array}\right)
$$

or

$$
\dot{\psi}(t)=A \psi(t)+g_{1}(\psi(t))+g_{2}(t, \psi(t))
$$


where $\psi=(u, \bar{v}), \bar{v}=v-1$, with

$$
\begin{aligned}
A & :=\left(\begin{array}{cr}
1-p & 0 \\
1 & 1
\end{array}\right), \\
g_{1}(z) & =\left(\begin{array}{c}
-u^{2}-p u \bar{v} \\
u \bar{v}+\bar{v}^{2}
\end{array}\right), g_{2}(t, z)=\left(\begin{array}{c}
(q-1) u \frac{e^{-2 t}}{1+e^{-2 t}} \\
0
\end{array}\right),
\end{aligned}
$$

$z=(u, \bar{v})$.

We remember $\lambda_{1}=1-p<0, \lambda_{2}=1, \xi_{1}=(p,-1), \xi_{2}=(0,1)$ from Section 2.5. The stable manifold of the linear part of $\left(\mathrm{EFS}_{p, 1}\right)$ is given by $S=\mathbb{R} \xi_{1}$, the unstable manifold by $U=\mathbb{R} \xi_{2}$. Let

$$
\begin{aligned}
& Q_{S}: \mathbb{R}^{2} \rightarrow S, \\
& Q_{U}: \mathbb{R}^{2} \rightarrow U,
\end{aligned}
$$

be the canonical projections. There exists $a>0$ so that $\left|Q_{S} x\right| \leq a|x|$, $\left|Q_{U} x\right| \leq a|x|$. Furthermore,

$$
Q_{S} e^{t A}=e^{t A} Q_{S}=e^{\lambda_{1} t} Q_{S}, \quad Q_{U} e^{t A}=e^{t A} Q_{U}=e^{\lambda_{2} t} Q_{U} .
$$

The solution $\psi$ of (7.1) satisfies for $\sigma, t \geq \tau>T_{0}$

$$
\psi(t)=e^{(t-\sigma) A} \psi(\sigma)+\int_{\sigma}^{t} e^{(t-s) A} g_{1}(\psi(s)) d s+\int_{\sigma}^{t} e^{(t-s) A} g_{2}(s, \psi(s)) d s
$$

and from a) we have $\psi(t) \rightarrow 0(t \rightarrow \infty)$. We apply $Q_{S}$ for $\sigma=\tau$ and $Q_{U}$ and get

$$
\begin{aligned}
\psi(t)=e^{\lambda_{1}(t-\tau)} Q_{S} \psi(\tau)+\int_{\tau}^{t} e^{\lambda_{1}(t-s)} Q_{S} g_{1}(\psi(s)) d s \\
\quad+\int_{\tau}^{t} e^{\lambda_{1}(t-s) A} Q_{S} g_{2}(s, \psi(s)) d s+e^{t-\sigma} Q_{U} \psi(\sigma) \\
\quad+\int_{\sigma}^{t} e^{t-s} Q_{U} g_{1}(\psi(s)) d s+\int_{\sigma}^{t} e^{t-s} Q_{U} g_{2}(s, \psi(s)) d s .
\end{aligned}
$$

Let $\delta>0$ be such that

$$
\frac{a(p+q) p}{p-1} \cdot \delta<1, \quad \frac{a(p+q) \delta}{1-\frac{a(p+q)}{p-1} \cdot \delta}<\min \left(\frac{p-1}{2}, 2\right)
$$

and let $\tau$ be so large that

$$
|\psi(t)| \leq \delta, \quad \frac{e^{-2 t}}{1+e^{-2 t}} \leq \delta \quad \text { for } t \geq \tau .
$$

The estimates

$$
\begin{array}{rlrl}
\left|Q_{U} \psi(\sigma)\right| \leq a \cdot \delta, & & \left|Q_{U} g_{1}(\psi(s))\right| \leq a(p+1) \delta^{2}, \\
& \left|Q_{U} g_{2}(\psi(s))\right| \leq a(q-1) e^{-2 s}
\end{array}
$$


show that we can let $\sigma \rightarrow+\infty$ in (7.2) and we get

$$
\begin{gathered}
\psi(t)=e^{\lambda_{1}(t-\tau)} Q_{S} \psi(\tau)+\int_{\tau}^{t} e^{\lambda_{1}(t-s)} Q_{S} g_{1}(\psi(s)) d s+\int_{\tau}^{t} e^{\lambda_{1}(t-s)} Q_{S} g_{2}(s, \psi(s)) d s \\
-\int_{t}^{\infty} e^{t-s} Q_{U} g_{1}(\psi(s)) d s-\int_{t}^{\infty} e^{t-s} Q_{U} g_{2}(s, \psi(s)) d s
\end{gathered}
$$

The next step is to deduce an estimate for $\psi$. We have for $Q=Q_{U}$ or $Q=Q_{S}$, with (7.4),

$$
\left.\begin{array}{rl}
\left|Q g_{1}(\psi(s))\right| & \leq a(p+1) \delta|\psi(s)| \\
\left|Q g_{2}(s, \psi(s))\right| & \leq a(q-1) \frac{e^{-2 t}}{1+e^{-2 t}}|\psi(s)| \leq a(q-1) \delta|\psi(s)|,
\end{array}\right\}
$$

so that (7.5) yields

$$
\begin{aligned}
|\psi(t)| & \leq e^{\lambda_{1} t} e^{-\lambda_{1} \tau}\left|Q_{S} \psi(\tau)\right|+a(p+q) \delta \int_{\tau}^{t} e^{\lambda_{1}(t-s)}|\psi(s)| d s \\
& +a(p+q) \delta \int_{t}^{\infty} e^{(t-s)}|\psi(s)| d s \\
& =: K e^{-\alpha t}+L \int_{\tau}^{t} e^{-\alpha(t-s)}|\psi(s)| d s+M \int_{0}^{\infty} e^{-\gamma s}|\psi(s+t)| d s
\end{aligned}
$$

with $\alpha:=-\lambda_{1}=p-1>0, \gamma:=1$. We may apply Lemma 7.1 , because

$$
\beta:=\frac{L}{\alpha}+\frac{M}{\gamma}=a(p+q)\left(\frac{1}{p-1}+1\right) \delta=\frac{a(p+q) p}{p-1} \cdot \delta<1,
$$

and we get

$$
|\psi(t)| \leq K^{*} e^{\left(\frac{L}{1-\beta}-\alpha\right) t}, \quad t \geq \tau
$$

Multiplying (7.5) by $e^{-\lambda_{1} t}$, we get

$$
\begin{aligned}
e^{-\lambda_{1} t} \psi(t) & =e^{-\lambda_{1} \tau} Q_{S} \psi(\tau)+\int_{\tau}^{t} e^{-\lambda_{1} s} Q_{S} g_{1}(\psi(s)) d s \\
& +\int_{\tau}^{t} e^{-\lambda_{1} s} Q_{S} g_{2}(s, \psi(s)) d s-e^{-\lambda_{1} t} \int_{t}^{\infty} e^{t-s} Q_{U} g_{1}(\psi(s)) d s \\
& -e^{-\lambda_{1} t} \int_{t}^{\infty} e^{t-s} Q_{U} g_{2}(s, \psi(s)) d s \\
& =: e^{-\lambda_{1} t} Q_{S} \psi(\tau)+\text { I }+ \text { II }+ \text { III }+ \text { IV }
\end{aligned}
$$

We want to show that the limits of all terms exist. Let us define $\varepsilon:=\frac{L}{1-\beta}$. From (7.3) and (7.7) we have

$$
\varepsilon<\frac{p-1}{2} \text { and } \varepsilon<2
$$


(I): The estimates

$$
e^{-\lambda_{1} s}\left|Q_{S} g_{1}(\psi(s))\right| \leq e^{-\lambda_{1} s} a(p+1) K^{* 2} e^{2(\varepsilon-\alpha) s}
$$

(with (7.8)) and $-\lambda_{1}+2 \varepsilon-2 \alpha=p-1+2 \varepsilon-2(p-1)=2 \varepsilon-(p-1)<0$ (with (7.9)) show that the integrand is integrable on $[\tau, \infty$ ).

(II): The estimates

$$
\begin{aligned}
e^{-\lambda_{1} s}\left|Q_{S} g_{2}(s, \psi(s))\right| & \leq e^{-\lambda_{1} s} a(q-1) e^{-2 s}|\psi(s)| \\
& \leq a(q-1) K^{*} e^{-\lambda_{1} s} e^{-2 s} e^{(\varepsilon-\alpha) s}
\end{aligned}
$$

(with (7.6) and (7.8)) and $-\lambda_{1}-2+\varepsilon-\alpha=-2+\varepsilon<0$ (with (7.9)) show that the integrand is also integrable on $[\tau, \infty)$.

(III):

$$
\begin{aligned}
e^{-\lambda_{1} t} \int_{t}^{\infty} e^{t-s}\left|Q_{U} g_{1}(\psi(s))\right| d s & \leq e^{p t} \int_{t}^{\infty} e^{-s} a(p+1)|\psi(s)|^{2} d s \\
& \leq e^{p t} \int_{t}^{\infty} e^{-s} a(p+1) K^{* 2} e^{2(\varepsilon-\alpha) s} d s \\
& <\frac{a(p+1) K^{* 2}}{-(-1+2(\varepsilon-\alpha))} e^{p t} e^{(-1+2(\varepsilon-\alpha)) t}
\end{aligned}
$$

(with (7.8)) and $p-1+2 \varepsilon-2 \alpha=p-1+2 \varepsilon-2(p-1)=2\left(\varepsilon-\frac{p-1}{2}\right)<0$ (with (7.9)) show that III $\rightarrow 0$.

(IV):

$$
\begin{aligned}
e^{-\lambda_{1} t} \int_{t}^{\infty} e^{t-s}\left|Q_{U} g_{2}(s, \psi(s))\right| d s & \leq e^{p t} \int_{t}^{\infty} e^{-s} a(q-1) e^{-2 s}|\psi(s)| d s \\
& \leq a(q-1) K^{*} e^{p t} \int_{t}^{\infty} e^{-s} \cdot e^{-2 s} e^{(\varepsilon-\alpha) s} d s \\
& <\frac{a(q-1) K^{*}}{-(-1+2+\varepsilon-\alpha)} e^{p t} e^{(-1-2+\varepsilon-\alpha) t}
\end{aligned}
$$

(with (7.8)) and $p-3+\varepsilon-\alpha=p-3+\varepsilon+1-p=\varepsilon-2<0$ (with (7.9)) show that IV $\rightarrow 0$.

Hence there exist $c_{1}, c_{2} \in \mathbb{R}$ such that

$$
\begin{aligned}
& e^{(p-1) t} u(t) \rightarrow c_{1}, \quad e^{(p-1) t}(v(t)-1) \rightarrow c_{2} \quad(t \rightarrow \infty), \\
& r^{p-1} u(\ln r) \rightarrow c_{1}, \quad r^{p-1}(v(\ln r)-1) \rightarrow c_{2} \quad(r \rightarrow \infty) .
\end{aligned}
$$

With (2.2.5),

$$
\begin{gathered}
r^{p-1} \phi^{p-1}(r)=r^{p-1} \frac{\left(1+r^{2}\right)^{\lambda / 2}}{r^{\lambda}} u(\ln r) v(\ln r) \rightarrow c_{1} \geq 0, \\
r \phi(r) \rightarrow c_{1}^{\frac{1}{p-1}}, \quad-r^{2} \cdot \phi^{\prime}(r)=r \phi(r) \cdot v(\ln r) \rightarrow c_{1}^{\frac{1}{p-1}} .
\end{gathered}
$$


Integration of $\left(\mathrm{M}_{p, \lambda}\right)$ gives

$$
-r^{2} \phi^{\prime}(r)=-r_{0}^{2} \phi^{\prime}\left(r_{0}\right)+\int_{r_{0}}^{r} \frac{s^{\lambda}}{\left(1+r^{2}\right)^{\lambda / 2}} \phi(s)^{p} d s>-r_{0}^{\prime} \phi^{\prime}\left(r_{0}\right)>0,
$$

which implies that $c_{1}>0$. Hence a) $\left.\rightarrow \mathrm{b}\right)$, c) with $c:=c_{1}^{\frac{1}{p-1}}$.

b) $\rightarrow$ a): With (2.2.4),

$$
\begin{aligned}
\begin{aligned}
u(t) & =\frac{r^{\lambda-1}}{\left(1+r^{2}\right)^{\lambda / 2}} \frac{\phi^{p}(r)}{-\phi^{\prime}(r)}=\frac{r^{\lambda-1}}{\left(1+r^{2}\right)^{\lambda / 2}} \frac{\frac{c^{p}}{r^{p}}(1+o(1))}{r^{2}}(1+o(1)) \\
& =-c^{p-1} \frac{r^{\lambda}}{\left(1+r^{2}\right)^{\lambda / 2}} \frac{r^{2}}{r \cdot r^{p}}(1+o(1))=-\frac{c^{p-1}}{r^{p-1}}(1+o(1)) \rightarrow 0, \\
v(t) & =r \frac{-\phi^{\prime}(r)}{\phi(r)}=\frac{-r^{2} \phi^{\prime}(r)}{r^{2} \phi(r)}=\frac{c(1+o(1))}{c(1+o(1))} \rightarrow 1 .
\end{aligned} \\
c) \rightarrow \text { a) is obvious. }
\end{aligned}
$$

We need to show the remaining assertions. For $q(t)<p$, we have $P_{2} \in \overline{S_{-}(t)}$ (see Section 2.6). Hence there exist the inverse function $t=t(u)$ and the function $\hat{v}(u)=v(t(u))$. Furthermore,

$$
\begin{aligned}
\hat{v}^{\prime}(u(t)) & =\frac{\dot{v}(t)}{\dot{u}(t)}=\frac{v(t)(-1+u(t)+v(t))}{u(t)(q(t)-u(t)-p v(t))} \\
& =\frac{v(t)}{q(t)-u(t)-p v(t)}\left(\frac{e^{-(p-1) t}(v(t)-1)}{e^{-(p-1) t} u(t)}+1\right) \\
& \rightarrow \frac{1}{1-p}\left(\frac{c_{2}}{c_{1}}+1\right) .
\end{aligned}
$$

This shows that the limit

$$
\lim _{u \rightarrow 0} \hat{v}^{\prime}(u)=\lim _{t \rightarrow \infty} \hat{v}^{\prime}(u(t))=\frac{1}{1-p}\left(\frac{c_{2}}{c_{1}}+1\right)=: L
$$

exists, and, by de l'Hospital's rule,

$$
\frac{c_{2}}{c_{1}}=\lim _{t \rightarrow \infty} \frac{v(t)-1}{u(t)}=L
$$

From this we get the equation

$$
L=\frac{1}{1-p}(L+1)
$$

which gives $L=-\frac{1}{p}$ and $c_{2}=-\frac{1}{p} \cdot c_{1}=-\frac{1}{p} c^{p-1}$. 


\section{8. $P_{3}^{+}$-solutions (solutions of slow decay)}

The general assumption of this section is again the one of Section 7 .

The following theorem characterizes the $P_{3}^{+}$-solutions, from which their existence will follow.

Theorem 8.1. Let $p, q>1$. The following conditions are equivalent:

a) $\phi$ is a $P_{3}^{+}$-solution.

b) We have

$$
\begin{aligned}
\phi(r) & =\left(\frac{1}{(p-1) \ln r}\right)^{\frac{1}{p-1}}(1+o(1)), \\
\phi^{\prime}(r) & =-\left(\frac{1}{(p-1) \ln r}\right)^{\frac{p}{p-1}} \cdot \frac{1}{r}(1+o(1))(r \rightarrow \infty) .
\end{aligned}
$$

c) We have

$$
\begin{aligned}
& u(t)=1-\frac{p}{p-1} \frac{1}{t}(1+o(1)), \\
& v(t)=\frac{1}{p-1} \frac{1}{t}(1+o(1))(t \rightarrow \infty) .
\end{aligned}
$$

d) There exists $t_{0}>T^{*}$ with $\varphi\left(t_{0}\right) \in G_{-}$.

e) There exists $t_{1}>T^{*}$ with $\varphi\left(t_{1}\right) \in W_{-}$.

In this case

$$
\lim _{u \rightarrow 1} \hat{v}^{\prime}(u)=\hat{v}^{\prime}(1)=-\frac{1}{p} .
$$

Proof. a) $\rightarrow$ c): Let $y(t):=\frac{1-u(t)}{v(t)}$ and $g(t):=(q-1) \frac{e^{-2 t}}{1+e^{-2 t}}$. Then

$$
\begin{aligned}
\dot{y} & =\frac{-v \dot{u}-(1-u) \dot{v}}{v^{2}}=-\frac{1}{v} u(1+g(t)-u-p v)-y(-1+u+v) \\
& =-u y+p u-\frac{u}{v} g(t)+y-u y+u-1 \\
& =(-2 u+1) y+(p+1) u-1-\frac{u}{v} g(t)=: r(t) y+b(t) .
\end{aligned}
$$

By a), $r(t) \rightarrow-1$. To determine the limit of $b$, we note

$$
\left(\frac{1}{v}\right)^{\cdot}=-\frac{\dot{v}}{v^{2}}=\frac{(1-u-v)}{v} \leq \frac{1}{v}, \text { hence } \frac{1}{v(t)} \leq K e^{t},
$$

for some constant $K>0$. Hence $b(t) \rightarrow p$. By Lemma 5.5 and Remark 2, $y(t) \rightarrow p$. Therefore $\left(\frac{1}{v}\right)^{\cdot}=y-1$ implies

$$
\begin{aligned}
v(t) & =\frac{1}{t} \frac{1}{\frac{1}{t} \frac{1}{v\left(t_{0}\right)}+\frac{1}{t} \int_{t_{0}}^{t}(y(s)-1) d s}=\frac{1}{t} \frac{1}{p-1}(1+o(1)), \\
1-u(t) & =v(t) y(t)=\frac{1}{t} \frac{p}{p-1}(1+o(1)),
\end{aligned}
$$


which is c).

c) $\rightarrow$ b) follows from (2.2.5).

b) implies a weaker form of c), namely

$$
\begin{aligned}
u(t) & =\frac{r^{\lambda-1}}{\left(1+r^{2}\right)^{\lambda / 2}} \frac{\phi^{p}(r)}{-\phi^{\prime}(r)} \\
& =\frac{r^{\lambda-1}}{\left(1+r^{2}\right)^{\lambda / 2}}\left(\frac{1}{(p-1) \ln r}\right)^{\frac{p}{p-1}}((p-1) \ln r)^{\frac{p}{p-1}} r(1+o(1)) \\
& =1+o(1) \\
v(t) & =r \frac{-\phi^{\prime}(r)}{\phi(r)}=r\left(\frac{1}{(p-1) \ln r}\right)^{\frac{p}{p-1}} \cdot \frac{1}{r}((p-1) \ln r)^{\frac{1}{p-1}}(1+o(1)) \\
& =\frac{1}{p-1} \frac{1}{t}(1+o(1))
\end{aligned}
$$

which already implies a).

d) $\rightarrow$ a): By Lemma 2.3, $\varphi(t) \in G_{-}$for $t \geq t_{0}$ and by Thieme's theorem, $\varphi$ tends to $P_{2}$ or $P_{3}^{+}$. However, any solution tending to $P_{2}$ must have slope $\hat{v}^{\prime}(0)=-\frac{1}{p}>-\frac{2}{p+1}$ (the slope of $\partial G_{-}$) by Theorem 7.2, which implies a).

a) $\rightarrow$ e): follows from the definition of $G_{-}$.

e) $\rightarrow$ d) is trivial because $W_{-} \subset G_{-}$.

Finally, let us consider $y_{1}:=t(y-p)$. We get

$$
\dot{y}_{1}=\frac{1}{t} y_{1}+t(r y+b)=\left(r+\frac{1}{t}\right) y_{1}+t(p r+b) .
$$

Because $r+\frac{1}{t}=(-2 u+1)+\frac{1}{t} \rightarrow 1$ and

$$
\begin{aligned}
t(p r+b) & =t\left(-2 p u+p+(p+1) u-1+\frac{p u}{v} g\right) \\
& =t(p-1)(1-u)+t \frac{p u}{v} g=p(1+o(1))+O\left(t^{2} e^{-2 t}\right)=p(1+o(1)),
\end{aligned}
$$

we have $y_{1}(t) \rightarrow b$ by Lemma 5.5 and Remark 2 . Hence

$$
\begin{aligned}
\lim _{u \rightarrow 1} \hat{v}(u) & =\lim _{t \rightarrow \infty} \frac{v(-1+u+v)}{u(1+g(t)-u-p v)}=\lim _{t \rightarrow \infty} \frac{\frac{-1+u}{v}+1}{u\left(\frac{g(t)}{v^{2}}+\frac{\frac{1-u}{v}-p}{t v}\right)} \\
& =\lim _{t \rightarrow \infty} \frac{-y+1}{u\left(\frac{g(t)}{v^{2}}+\frac{y_{1}}{t v}\right)}=\frac{-p+1}{1(0+(p-1) p)}=-\frac{1}{p}
\end{aligned}
$$

\section{Global behaviour of the solutions and their radius and mass}

The aim of this section is to investigate the question which $F-, E-, M$ solutions have finite or infinite radius and mass. We give numerous initial 
conditions in $\mathbb{R}^{+} \times \mathbb{R}^{+}$, for which the corresponding solutions have the desired properties. These initial conditions can be transformed back to conditions for $\phi\left(r_{0}\right), \phi^{\prime}\left(r_{0}\right)$ with the equations (2.2.5). The total mass of the stellar dynamic model induced by a solution $\phi$ of $\left(\mathrm{M}_{p, \lambda}\right)$ is given by

$$
\begin{aligned}
M & \left.=\bar{c} \int_{R_{-}}^{R} \frac{r^{\lambda}}{\left(1+r^{2}\right)^{\lambda / 2}} \phi(r)^{p} d r=-\bar{c} \int_{R_{-}}^{R}\left(r^{2} \phi^{\prime}(r)\right)^{\prime}\right) d r \\
& =\bar{c} \lim _{r \downarrow R_{-}} r^{2} \phi^{\prime}(r)+\bar{c} \lim _{r \uparrow R} r^{2}\left(-\phi^{\prime}(r)\right)=: L_{-}+L,
\end{aligned}
$$

with $\bar{c}$ as in (1.4).

Let us remark immediately that the limit $L_{-}$is always finite-this follows for the $F$-solutions from Section 3, for the $E$-solutions from Theorem 4.1, and for the $M$-solutions from Theorems 5.1, 5.7, 5.8 (note that $-\frac{\lambda}{p+1}-1+$ $2>0 \Leftrightarrow q<p$ ) and 5.11 (note that $r^{2} \phi^{\prime} \rightarrow 0$ for $r \rightarrow 0$ by $(2.2 .5)$ ). Hence $M<\infty \Longleftrightarrow L<\infty$.

The following theorem characterizes the solutions $\phi$ with finite and infinite radius and mass by their images $\varphi$ in $\mathbb{R}^{+} \times \mathbb{R}^{+}$.

Theorem 9.1. We have the following three equivalences:

a) $R<\infty \Longleftrightarrow \varphi \rightarrow \varnothing$. In this case $M<\infty$.

b) $R=\infty, M<\infty \Longleftrightarrow \phi$ is a $P_{2}$-solution, that is, $\varphi \rightarrow P_{2}$.

c) $R=\infty, M=\infty \Longleftrightarrow \phi$ is a $P_{3}^{+}$-solution, that is, $\varphi \rightarrow P_{3}^{+}$.

Proof. We have $R<\infty \Longrightarrow M<\infty \Longleftrightarrow L<\infty$. Hence a) follows from Theorem 6.1. Parts b) and c) follow from Theorems 7.2 and 8.1 in connection with Corollary 6.2.

\subsection{The case $q \leq \frac{p+1}{2}$}

Theorem 9.2. Let $q \leq \frac{p+1}{2}$. If $\varphi$ is any solution for which $\varphi\left(t_{0}\right) \in G_{-}$, then $\varphi(t) \rightarrow P_{3}^{+}(t \rightarrow \infty)$. Consequently, all $M$ - and E-solutions, and all $F$-solutions for which $\varphi\left(t_{0}\right) \in G_{-}$for some $t_{0}$, have $R=\infty, M=\infty$.

Proof. The first assertion follows from Theorem 8.1. The condition $\varphi\left(t_{0}\right) \in$ $G_{-}$is satisfied for $M$-solutions because $\varphi(t) \rightarrow P_{4}(t \rightarrow-\infty)$ by Theorem 5.8 , and for $E$-solutions by Theorem 4.1 in the case $q<\frac{p+1}{2}$, and by the remarks following Lemma 5.10 in the case $q=\frac{p+1}{2}$.

Remark 4. The part on $E$-solutions is known (see Section 9.4). 


\section{2. $M$-solutions for $q>\frac{p+1}{2}$}

We are going to show that in this case the three possibilities a), b), c) of Theorem 9.1 all occur. To this end, it is useful to remark that for any solution we have for $q>\frac{p+1}{2}$ :

(i) If there exists $t_{0}<T^{*}$ with $\varphi\left(t_{0}\right) \in G_{-} \cup G_{0}$, then $\varphi$ corresponds to an $M$-solution.

(ii) If there exists $t_{1} \in \mathbb{R}$ with $v\left(t_{1}\right)>1$, then $\varphi \rightarrow \varnothing(t \rightarrow \infty)$.

(iii) If there exists $t_{2}>T^{*}$ with $\varphi\left(t_{2}\right) \in G_{-}$, then $\varphi \rightarrow P_{3}^{+}(t \rightarrow \infty)$.

In fact, (i) implies with Lemma 2.3 that $\varphi\left(t_{0}\right) \in G_{-}$for all $t \leq t_{0}$. Because $L^{-}(\varphi)$ is bounded, by Thieme's theorem, $\varphi \rightarrow P_{i}$ or $P_{3}^{-}(t \rightarrow-\infty)$ where $i=2$ in the case $q \geq p$ and $i=4$ in the case $\frac{p+1}{2}<q<p$. Convergence to $P_{3}^{-}$is not possible because $q>\frac{p+1}{2}$, and $\varphi$ is an $M$-solution by Theorems 5.1, 5.7 or 5.8. (ii) follows from Theorem 6.1 and (iii) from Theorem 8.1.

Furthermore, we need the following lemma.

Lemma 9.3. Let $q>\frac{p+1}{2}$. Then there exists $\varepsilon^{*}>0, T_{1}<T^{*}$ such that for all $\left(u_{0}, v_{0}\right) \in G_{0}$ with $\left|\left(u_{0}, v_{0}\right)-P_{2}\right|<\varepsilon, 0<\varepsilon<\varepsilon^{*}$ and for all $t_{0} \leq T_{1}$

there exists $t_{1}>t_{0}$ such that $v\left(t_{1}, t_{0}, u_{0}, v_{0}\right)>1$.

Proof. For $0<\varepsilon<1$ we have $\left(u_{0}, v_{0}\right)=\left(\varepsilon \frac{p+1}{2}, 1-\varepsilon\right) \in G_{0}$. We let

$$
\begin{aligned}
x(t) & :=\frac{1}{\varepsilon} u\left(t, t_{0}, \varepsilon \frac{p+1}{2}, 1-\varepsilon\right) \\
y(t) & :=\frac{1}{\varepsilon}\left[1-v\left(t, t_{0}, \varepsilon \frac{p+1}{2}, 1-\varepsilon\right)\right],
\end{aligned}
$$

and we are going to specify $t_{1}, t_{0}$. The functions $x, y$ satisfy

$$
\begin{aligned}
& \dot{x}=x[q(t)-\varepsilon x-p(1-\varepsilon y)], \\
& \dot{y}=-(1-\varepsilon y)(x-y) .
\end{aligned}
$$

We consider the limit case $\varepsilon \rightarrow 0$ and arrive at

$$
\begin{aligned}
& \dot{x}=x[q(t)-p]=x(q-p-g(t)), \\
& \dot{y}=-x+y, \text { where } g(t)=\lambda \frac{e^{2 t}}{1+e^{2 t}},
\end{aligned}
$$


so that for $t \geq t_{0}$

$$
\begin{aligned}
x(t) & =\frac{p+1}{2} e^{(q-p)\left(t-t_{0}\right)}\left(\frac{1+e^{2 t_{0}}}{1+e^{2 t}}\right)^{\frac{\lambda}{2}}, \\
y(t) & =e^{t-t_{0}}\left(1-\int_{t_{0}}^{t} x(s) e^{-\left(s-t_{0}\right)} d s\right) \\
& =e^{t-t_{0}}\left(1-\int_{0}^{t-t_{0}} x\left(s+t_{0}\right) e^{-s} d s\right) \\
& =e^{t-t_{0}}\left(1-\frac{p+1}{2} \int_{0}^{t-t_{0}} e^{(q-p-1) s}\left(\frac{1+e^{2 t_{0}}}{1+e^{2 s} e^{2 t_{0}}}\right)^{\frac{\lambda}{2}} d s\right) .
\end{aligned}
$$

If we fix $\tau:=t-t_{0}>1$ and let $t_{0} \rightarrow-\infty$, the above integral converges to

$$
\int_{0}^{\tau} e^{(q-p-1) s} d s
$$

Hence there exists $T_{1}<T^{*}$ such that for $t_{0} \leq T_{1}$

$$
\int_{0}^{\tau} e^{(q-p-1) s}\left(\frac{1+e^{2 t_{0}}}{1+e^{2 s} e^{2 t_{0}}}\right)^{\frac{\lambda}{2}} d s>\int_{0}^{\tau-1} e^{(q-p-1) s} d s .
$$

On the other hand we have

$$
\frac{p+1}{2} \int_{0}^{\infty} e^{(q-p-1) s} d s= \begin{cases}\infty & \text { for } q \geq p+1 \\ \frac{1}{p+1-q}>1 & \text { for } \frac{p+1}{2}<q<p+1\end{cases}
$$

Therefore we may assume that $\tau$ had been chosen so large that

$$
\frac{p+1}{2} \int_{0}^{\tau-1} e^{(q-p-1) s} d s>1
$$

Then for $t_{1}:=\tau+t_{0}$ we have $y\left(t_{1}\right)<0$ and, by continuity, $v\left(t_{1}\right)>1$ for all sufficiently small $\varepsilon>0$.

Theorem 9.4. Let $q>\frac{p+1}{2}$.

(a) If $\left(u_{0}, v_{0}\right) \in G_{0}$ is close to $P_{2}$ and $t_{0}<T_{1}$ as in Lemma 9.3, then the $M$-solution $\varphi\left(\cdot, t_{0}, u_{0}, v_{0}\right)$ has $R<\infty$ and $M<\infty$.

(b) If $\left(u_{1}, v_{1}\right) \in G_{-} \cup G_{0}$, then the $M$-solution $\varphi\left(\cdot, T^{*}, u_{1}, v_{1}\right)$ has $R=\infty$ and $M=\infty$.

(c) For $t_{0},\left(u_{0}, v_{0}\right)$ from (a) and $\left(u_{1}, v_{1}\right)$ from (b), there exists some $s \in(0,1)$ such that for $\left(t_{s}, u_{s}, v_{s}\right)=(1-s)\left(t_{0}, u_{0}, v_{0}\right)+s\left(T^{*}, u_{1}, v_{1}\right)$, $\varphi\left(\cdot, t_{s}, u_{s}, v_{s}\right)$ is an $M$-solution with $R=\infty$ and $M<\infty$.

Proof. We apply Theorem 9.1. (a) follows from the proceeding remarks (i) and (ii).

(b): Lemma 2.3 shows that $\varphi\left(t, T^{*}, u_{1}, v_{1}\right) \in G_{-}$for $t \neq T^{*}$, and remarks (i) and (iii) apply. 
(c): We let $Q(s):=\left(t_{s}, u_{s}, v_{s}\right)=(1-s)\left(t_{0}, u_{0}, v_{0}\right)+s\left(T^{*}, u_{1}, v_{1}\right): s \in$ $[0,1] \longmapsto\left[t_{0}, T^{*}\right] \times G_{-} \cup G_{0}$ and define the sets

$$
\begin{aligned}
& A:=\{s \in[0,1] ; \varphi(\cdot, Q(s)) \text { satisfies }(i) \text { and }(i i)\}, \\
& B:=\{s \in[0,1] ; \varphi(\cdot, Q(s)) \text { satisfies }(i) \text { and }(i i i)\} .
\end{aligned}
$$

We have $0 \in A, 1 \in B$. Because $\varphi$ depends continuously on the initial data, $A$ and $B$ are disjoint, non-empty and relatively open in $[0,1]$. Because $[0,1]$ is connected, there is some $s_{0} \in(0,1)$ which is not in $A \cup B$. On the other hand, $Q\left(s_{0}\right) \in\left(t_{0}, T^{*}\right) \times G_{-} \cup G_{0}$ and therefore $\varphi\left(\cdot, Q\left(s_{0}\right)\right)$ satisfies (i) but not (ii) and (iii), and hence necessarily tends to $P_{2}$. This proves (c).

\subsection{F-solutions}

Our first result in this section is that for all $p, q>1$ the three possibilities a), b), c) of Theorem 9.1 all occur. We need the following lemma.

Lemma 9.5. Let $p, q>1$. There exists $\eta>0$ such that for all $v_{0} \in(0, \eta)$ and $t_{0}>T^{*}$ there exists $t_{1}>t_{0}$ such that $\varphi\left(t_{1}, t_{0}, q, v_{0}\right) \in G_{-}$.

Proof. If $q<\frac{p+1}{2},\left(q, v_{0}\right) \in G_{-}$for small $v_{0}$ and the assertion is obvious for all $t_{0}$. Therefore we may assume $q \geq \frac{p+1}{2}$. We define $q_{0}:=q\left(t_{0}\right)<\frac{p+1}{2}$. We consider $\left(\mathrm{EF}_{p, q_{0}}\right)$ and the $F$-solution $\bar{\varphi}_{2}$ with $\bar{\varphi}_{2}(t) \rightarrow P_{2}$ (see [4, Theorem 5.7 , case $n>3 m+5])$. We may assume $\bar{u}_{2}\left(t_{0}\right)=q\left(\left(\mathrm{EF}_{p, q_{0}}\right)\right.$ is autonomous $)$ and define $\bar{v}_{2}\left(t_{0}\right)=: \eta$. Let $v_{0}<v_{1}<\eta$. From [4, Theorem 5.7] we deduce that the solution $\bar{\varphi}\left(\cdot, t_{0}, q, v_{1}\right)$ of $\left(\mathrm{EF}_{p, q_{0}}\right)$ stays below $\bar{\varphi}_{2}$ and there exist $\bar{t}_{2}>\bar{t}_{1}>t_{0}$ such that

$$
\bar{\varphi}\left(\bar{t}_{1}\right) \in G_{0} \text { and } \bar{\varphi}(t) \in S\left(p, q_{0}\right) \text { on }\left[t_{0}, \bar{t}_{2}\right) .
$$

Hence $\hat{\bar{v}}=\hat{\bar{v}}(u)$ exists on $\left[\bar{u}_{2}\left(t_{2}\right), q\right]$ with graph $\hat{\bar{v}} \subset S\left(p, q_{0}\right)_{-}$and satisfies

$$
\hat{\bar{v}}^{\prime}(u)=\frac{\hat{\bar{v}}(u)(-1+u+\hat{\bar{v}}(u))}{u\left(q_{0}-u-p \hat{\bar{v}}(u)\right)}=: \bar{k}(u, \hat{\bar{v}}(u)) .
$$

Now consider the $F$-solution $\varphi\left(\cdot, t_{0}, q_{0}, v_{0}\right)$ of $\mathrm{MS}_{p, \lambda}$. We want to show: there exists $t_{1}>t_{0}$ with $\varphi\left(t_{1}\right) \in G_{0}$ (the conclusion then follows from Lemma 2.3). We may assume that $\hat{v}$ also exists on $\left[\bar{u}\left(t_{2}\right), q\right]$ and graph $\hat{v} \subset S\left(p, q_{0}\right)_{-}$, because otherwise $\varphi$ would have crossed $S\left(p, q_{0}\right)_{0}$ and hence $G_{0}$ sometime before (see Section 2.6) and we are done. $\hat{v}$ satisfies

$$
\hat{v}^{\prime}(u)=\frac{\hat{v}(u)(-1+u+\hat{v}(u))}{u(q(t(u))-u-p \hat{v}(u))}=: k(u, \hat{v}(u)) .
$$

Because $t(u)>t_{0}$ for $u<q_{0}$, we have $q(t(u))<q\left(t_{0}\right)=q_{0}$ and

$$
k(u, v)>\bar{k}(u, v) \text { in } S\left(p, q_{0}\right)_{-} .
$$

Hence $\hat{v}(u)<\hat{\bar{v}}(u)$ on $\left[\bar{u}_{2}\left(t_{2}\right), q\right]$, and because $\bar{\varphi}$ crosses $G_{0}, \varphi$ crosses $G_{0}$ as well. 
Theorem 9.6. Let $q, p>1$. Then:

(a) For $\left(u_{0}, v_{0}\right)$ with $u_{0} \geq q, v_{0} \geq 1$ and all $t_{0}$ the F-solution $\varphi\left(\cdot, t_{0}, u_{0}, v_{0}\right)$ tends to $\varnothing$ and has $R<\infty$ and $M<\infty$.

(b) For $v_{0}<\eta$ as in Lemma 9.5 and for $t_{0}>T^{*}$ the F-solution $\varphi\left(\cdot, t_{0}, q, v_{0}\right)$ tends to $P_{3}^{+}$and has $R=\infty$ and $M=\infty$.

(c) There exists $v_{0} \in[\eta, 1]$ such that for $t_{0}>T^{*}$ the $F$-solution $\varphi\left(\cdot, t_{0}, q, v_{0}\right)$ tends to $P_{2}$ and has $R<\infty$ and $M=\infty$.

Proof. (a) and (b) follow from Theorem 3.1, Lemma 9.5 and Theorem 8.1.

As for (c), the sets

$$
\begin{aligned}
& A:=\left\{v_{0} \in(0,1]: \exists t>t_{0} \text { with } v\left(t, t_{0}, q, v_{0}\right)>1\right\} \\
& B:=\left\{v_{0} \in(0,1]: \exists t>t_{0} \text { with } \varphi\left(t, t_{0}, q, v_{0}\right) \in G_{-}\right\}
\end{aligned}
$$

are disjoint, non-empty and relatively open in $(0,1]$. Because $(0,1]$ is connected, the complement of $A \cup B$ in $(0,1]$ is non-empty and contains $v_{0}$ for which $\varphi\left(\cdot, t_{0}, q, v_{0}\right)$ necessarily tends to $P_{2}$.

Part b) of the following theorem is inspired by the work of W.-M. Ni and S. Yotsutani [24]. For $R_{0}>0$ and $\alpha>0$ we denote by $\phi\left(\cdot, R_{0}, \alpha\right)$ the $F$-solution with $\phi\left(R_{0}\right)=\alpha$ and $\phi^{\prime}\left(R_{0}\right)=0$.

Theorem 9.7. For every $R_{0}>0$, there exist constants $\alpha_{0}=\alpha_{0}\left(R_{0}, p, q\right)$ and $\alpha_{1}=\alpha_{1}\left(R_{0}, p, q\right)$ such that

a) $\phi\left(\cdot, R_{0}, \alpha\right)$ has $R<\infty$ for $\alpha \geq \alpha_{0}$.

b) $\phi\left(\cdot, R_{0}, \alpha\right)$ has $R=\infty$ for $\alpha \leq \alpha_{1}$.

Proof. a) The function $z(t):=\phi\left(\frac{1}{t}, R_{0}, \alpha\right)$ defined on $\left(\frac{1}{R}, t_{0}\right), t_{0}:=\frac{1}{R_{0}}$ is a solution of

$$
z^{\prime \prime}(t)+f_{\lambda}(t) z^{p}(t)=0, \text { where } f_{\lambda}(t):=\frac{1}{t^{2}\left(1+t^{2}\right)^{\frac{\lambda}{2}}} .
$$

It is sufficient to prove: there exists $t_{1} \in\left(\frac{1}{R}, t_{0}\right]$ such that

$$
z^{\prime}\left(t_{1}\right) \geq \frac{z\left(t_{1}\right)}{t_{1}}
$$

i.e., the tangent line passing through $\left(t_{1}, z\left(t_{1}\right)\right)$ has a zero in $\left[0, t_{1}\right)$. Because $z^{\prime \prime}<0$, this implies that $z$ has a zero in $\left(0, t_{0}\right)$ and $R<\infty$ follows.

If (9.2) were not true, we would have

$$
\frac{z^{\prime}(t)}{z(t)}<\frac{1}{t} \text { and hence } \frac{z(t)}{t}>\frac{\alpha}{t_{0}} \text { in }\left(\frac{1}{R}, t_{0}\right)
$$

by integration. Integrating (9.1) once, we obtain that

$$
\begin{aligned}
z^{\prime}(t) & =\int_{t}^{t_{0}} \frac{1}{s^{2}\left(1+s^{2}\right)^{\frac{\lambda}{2}}} z^{p}(s) d s \\
& \geq \frac{\alpha^{p}}{t_{0}^{p}} \int_{t}^{t_{0}} s^{p} f_{\lambda}(s) d s .
\end{aligned}
$$


Now there exists $t_{1} \in\left(0, t_{0}\right)$ such that $\alpha_{0}:=\left[\inf _{t \in\left(0, t_{0}\right)} \frac{t_{0}^{p}}{t \int_{t}^{t_{0}} s^{p} f_{\lambda}(s) d s}\right]^{\frac{1}{p-1}}=$ $\left[\frac{t_{0}^{p}}{t_{1} \int_{t_{1}}^{t_{0}} s^{p} f_{\lambda}(s) d s}\right]^{\frac{1}{p-1}}$. Then, if $\alpha>\alpha_{0}$, we would have $z^{\prime}\left(t_{1}\right)>\frac{z\left(t_{1}\right)}{t_{1}}$, which is a contradiction. This proves a).

b) We assume $R<\infty$,i.e. $\phi(R)=0$. By the Pohozaev-type identity [24, Theorem 1, p5.] we have for $n=3$ that

$$
\begin{gathered}
\frac{1}{2} R^{3} \phi^{\prime 2}(R)=\int_{R_{0}}^{R}\left[\frac{5-p}{2(p+1)} K(r)+\frac{1}{p+1} r K^{\prime}(r)\right] \phi^{p+1}(r) r^{2} d r \\
\quad+\frac{R_{0}^{3} K\left(R_{0}\right)}{p+1} \alpha^{p+1} \\
=\alpha^{p+1}\left\{\frac{R_{0}^{3} K\left(R_{0}\right)}{p+1}-\int_{R_{0}}^{R} G(r)\left[\frac{\phi(r)}{\alpha}\right]^{p+1} d r\right\}
\end{gathered}
$$

where

$$
G(r)=\frac{1}{2(p+1)}\left[p-1+\frac{p-2 \lambda-1}{r^{2}}\right] \frac{r^{\lambda+2}}{\left(1+r^{2}\right)^{\frac{\lambda}{2}+1}}
$$

so that

$$
G(r) \rightarrow \frac{p-1}{2(p+1)}=: G_{\infty}>0 \text { as } r \rightarrow \infty
$$

Let $R_{1} \geq R_{0}$ such that $G(r) \geq \frac{G_{\infty}}{2}$ if $r \geq R_{1}$ and let $M:=\sup _{r \geq 0}|G(r)|<$ $\infty$. Since $\phi(r) \leq \alpha$, we have

$$
\begin{aligned}
r^{2} \phi^{\prime}(r) & =-\int_{R_{0}}^{r} s^{2} K(s) \phi^{p}(s) d s \geq-\alpha^{p} \int_{R_{0}}^{r} s^{2} K(s) d s, \\
\phi^{\prime}(r) & \geq-\frac{\alpha^{p}}{r^{2}} \int_{R_{0}}^{r} s^{2} K(s) d s
\end{aligned}
$$

and hence

$$
\phi(r) \geq \alpha-\alpha^{p} \int_{R_{0}}^{r} \frac{1}{t^{2}} \int_{R_{0}}^{t} s^{2} K(s) d s d t
$$

or

$$
\frac{\phi(r)}{\alpha} \geq 1-\alpha^{p-1} g(r) \text { for } R_{0}<r<R
$$

where $g(r):=\int_{R_{0}}^{r} \frac{1}{t^{2}} \int_{R_{0}}^{t} s^{2} K(s) d s d t$. We now fix

$$
R_{2}:=R_{1}+\frac{2^{p+2}}{G_{\infty}}\left[\frac{R_{0}^{3} K\left(R_{0}\right)}{p+1}+M\left(R_{1}-R_{0}\right)\right] .
$$

Since $g \in C\left(\left[R_{0}, \infty\right)\right)$ there exists $\alpha_{1}>0$ such that for all $\alpha \in\left(0, \alpha_{1}\right]$

$$
1-\alpha^{p-1} g(r) \geq \frac{1}{2}, \text { on }\left[R_{0}, R_{2}\right],
$$


which in particular implies that $R>R_{2}, \alpha \in\left(0, \alpha_{1}\right]$. From (9.4)-(9.7) we obtain for $\alpha \leq \alpha_{1}$

$$
\begin{aligned}
\frac{1}{2} R^{3} \phi^{\prime 2}(R) & <\alpha^{p+1}\left\{\frac{R_{0}^{3} K\left(R_{0}\right)}{p+1}+\int_{R_{0}}^{R_{1}} M[1]^{p+1} d r-\int_{R_{1}}^{R_{2}} \frac{G_{\infty}}{2}\left[\frac{1}{2}\right]^{p+1} d r\right\} \\
& =\alpha^{p+1}\left\{\frac{R_{0}^{3} K\left(R_{0}\right)}{p+1}+M\left(R_{1}-R_{0}\right)-\frac{G_{\infty}}{2^{p+2}}\left(R_{2}-R_{1}\right)\right\} \\
& =0
\end{aligned}
$$

by the choice of $R_{2}$. This is a contradiction and $R=\infty$ follows.

\subsection{Known result on E-solutions}

For the sake of completeness, we collect the known results on the $E$ solutions which are relevant in our context. For $\alpha>0$ we denote by $\phi(\cdot, \alpha)$ the solution $\phi$ of $\left(M_{p, \lambda}\right)$ with $\phi(0)=\alpha$.

Theorem 9.8. a) For $q>\frac{p+1}{2}$ there exists some $\alpha_{0} \in(0, \infty)$, such that

1. ) for $\alpha>\alpha_{0}, \phi(\cdot, \alpha)$ has $R<\infty$ and $M<\infty$,

2. ) $\phi\left(\cdot, \alpha_{0}\right)$ has $R=\infty$ and $M<\infty$,

3. ) for $\alpha \in\left(0, \alpha_{0}\right) \phi(\cdot, \alpha)$ has $R=\infty$ and $M=\infty$.

b) For $q \leq \frac{p+1}{2}, \phi(\cdot, \alpha)$ has $R=\infty, M=\infty$ for all $\alpha>0$.

A number of authors contributed to this theorem in connection with a study of conjectures of Matukuma's in the case $\lambda=2$ [24, p. 2]. For example, W.-M. Ni and $S$. Yotsutani proved a) 1.) for large $\alpha>0,3$.) for all $p, q>1$ for small $\alpha$, and that for all $\alpha>0, \phi(\cdot, \alpha)$ has $R=\infty$ if $q \leq \frac{p+1}{2}$ and $M=\infty$ if $q<\frac{p+1}{2}$ [24, Theorems 2, 5 and 6]; see Y. Li and W.-M. Ni for results related to a) 3.) for $\lambda \geq 2$ [16, Theorem 3.20].

The existence of $E$-solutions with $R=\infty$ and $M<\infty$ for $q>\frac{p+1}{2}$ was proven by Y. Li and W.-M. Ni [17, Theorem 2.1]; their uniqueness by E. Yanagida [36]. Y. Li and W.-M. Ni also proved the precise asymptotics of the $E$-solutions at $r=\infty$ [16, Theorem 2.41], while Y. Li presented the asymptotic expansions of $E$-solutions with $R=\infty$ and $M=\infty$ for all $p, q>1$ in [14]. The result in its above final form was given by N. Kawano, E. Yanagida and S. Yotsutani [12, Theorem 5.2].

Remark 5. We do not know whether separation results analogous to Theorem 9.8 hold for $F$ - and $M$-solutions. 


\section{Appendix}

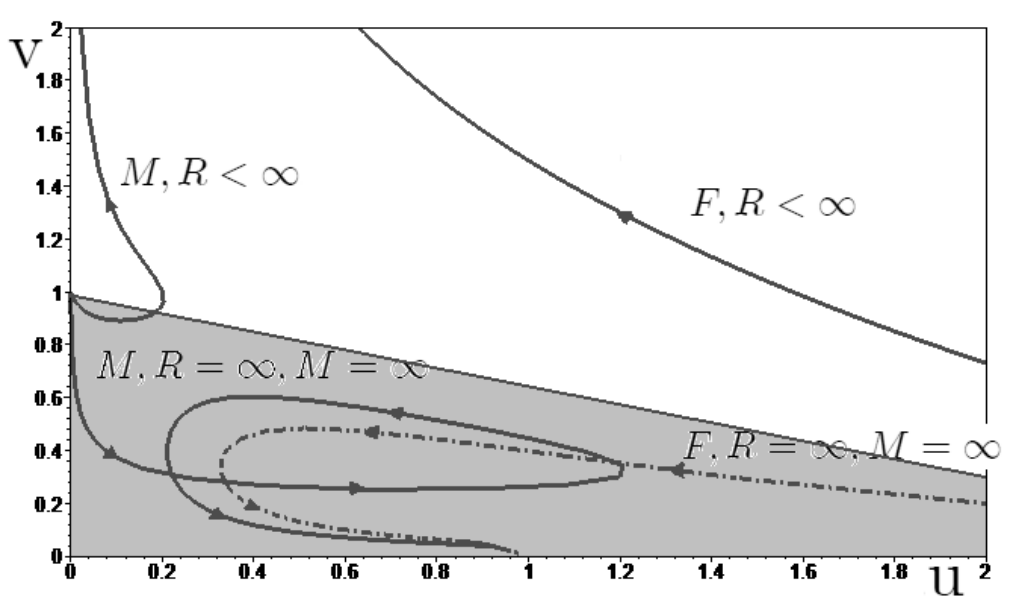

Fig. 1. Case $q>p(q=3, p=2)$. Illustration of Lemma 2.3 and Theorems 3.1, 9.6 (a), (b), 5.1 and 8.1. Note that the shaded region is the $G^{-}$(definition before Lemma 2.3) for Figure 1-4.

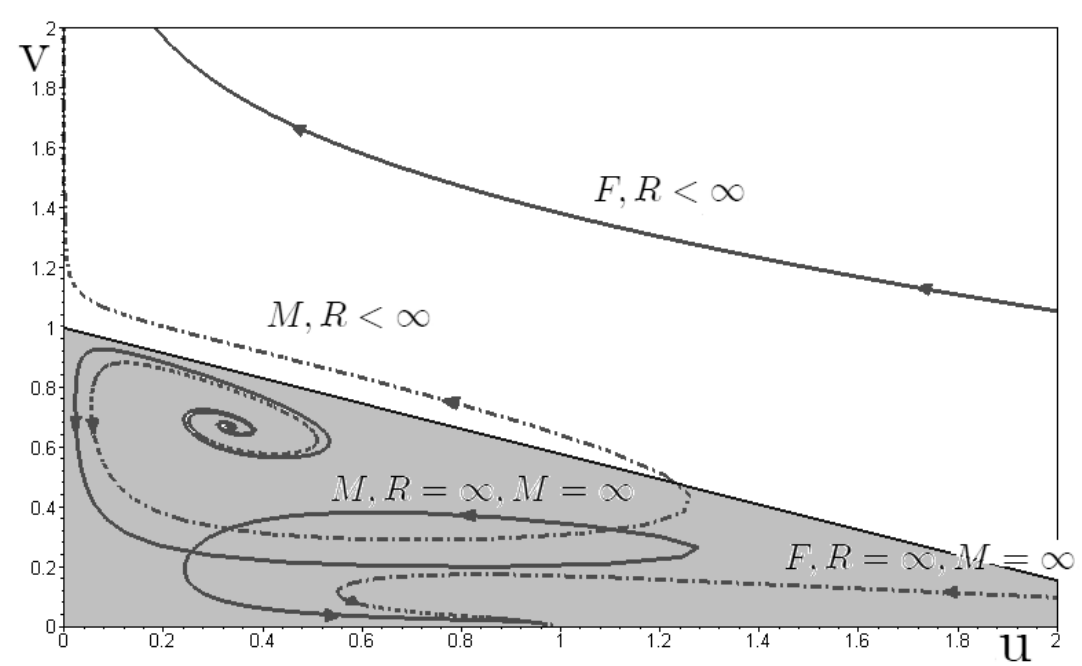

Fig. 2. Case $\frac{p+1}{2}<q<p(q=3, p=4)$. Illustration of Lemma 2.3 and Theorems 3.1, 9.6 (a), (b), 5.8 and 9.4 (a), (b). 


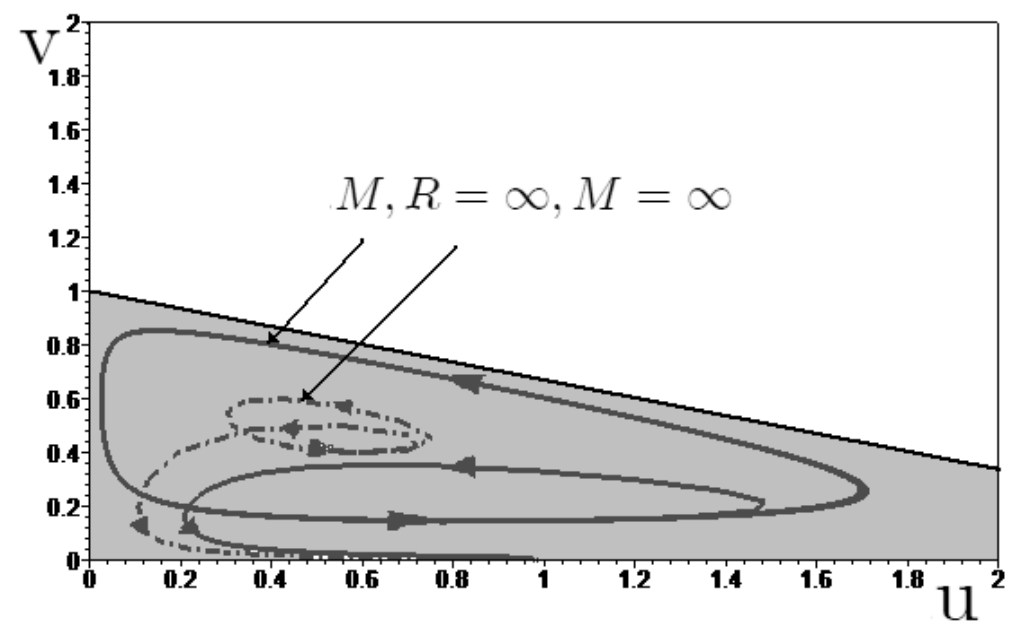

Fig. 3. Case $q=\frac{p+1}{2}(q=3, p=5)$. Illustration of Lemma 2.3 and Theorem 5.11.

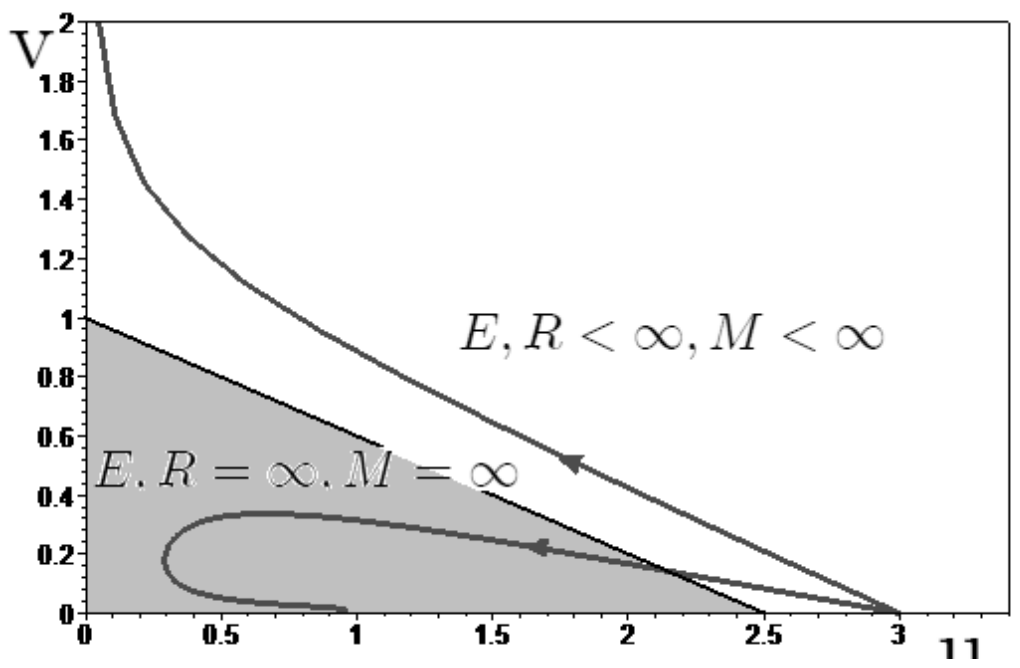

Fig. 4. Case $q>\frac{p+1}{2}(q=3, p=4)$. Illustration of Lemma 2.3 and Theorems 4.1, 8.1, and 9.8 . 
Acknowledgements. The authors are grateful to the Mathematisches Forschungs-Institut, Oberwolfach, Germany, and its Director, Prof. H.M. Greuel, for the grant of a stay of two weeks in Oberwolfach in November, 2006 within the program "Research in Pairs". The first author wants to acknowledge an encouraging correspondence with Prof. Zhien Ma, Xi'an Jiatong University, Xi'an, China. His former student Wolfgang Prenninger did the first steps in extending the paper [4] to the Matukuma equation. He wishes to thank the PDE research group at the Hunan Normal University, Changsha, China for their hospitality during his visit in July/August 2007 and the Deutsche Forschungsgemeinschaft for the travel grant (GZ: BA747/16-1). The second author was supported in part by University of Iowa International Programs, University of Iowa Office of the Vice President for Research, Stanley-UI Support Organization and Hunan Normal University Xiao-Xiang Fund and is grateful for the support.

\section{References}

1. Batt, J.: Steady state solutions of the relativistic Vlasov-Poisson system. Proceedings of the Fifth Marcel Grossmann Meeting on General Relativity, Part $B$ (Perth, 1988), World Sci. Publ., Teaneck, NJ, 1235-1247 (1989)

2. Batt, J., Faltenbacher, W., Horst, E.: Stationary spherically symmetric models in stellar dynamics. Arch. Rational Mech. Anal. 93, 159-183 (1986)

3. Batt, J., Morrison, P. J., Rein, G.: Linear stability of stationary solutions of the Vlasov-Poisson system in three dimensions. Arch. Rational Mech. Anal. 130, no. 2, 163-182 (1995)

4. Batt, J., Pfaffelmoser, K.: On the radius continuity of the models of polytropic gas spheres which correspond to the positive solutions of the generalized Emden-Fowler equation. Math. Methods Appl. Sci. 10, 499-516 (1988)

5. Bellman, R.: Stability Theory of Differential Equations. McGraw-Hill, New York-Toronto-London, 1953

6. Camm, G.L.: Self-gravitating star system II. Monthly Notices Royal Astronomical Society 112, 155-176 (1952)

7. Emden, R.: Gaskugeln. Leipzig und Berlin: Teubner 1907

8. Guo, Y.: Variational method for stable polytropic galaxies. Arch. Rational Mech. Anal. 150, 209-224 (1999)

9. Guo, Y., Rein, G.: Existence and stability of Camm type steady states in galactic dynamics. Indiana Univ. Math. J. 48, 1237-1255 (1999)

10. Guo, Y., Rein, G.: Stable steady states in stellar dynamics. Arch. Rational Mech. Anal. 147, 225-243 (1999)

11. Heinzle, J.M., Rendall, A.D., Uggla, C.: Theory of Newtonian self-gravitating stationary spherically symmetric systems. Math. Proc. Cambridge Philos. Soc. 140, 177-192 (2006)

12. Kawano, N., Yanagida, E., Yotsutani, S.: Structure theorems for positive radial solutions to $\triangle u+K(|x|) u^{p}=0$ in $\mathbf{R}^{n}$. Funkcial. Ekvac. 36, no. 3, 557-579 (1993)

13. Kwong,M.K., Li,Y.: Uniqueness of radial solutions of semilinear elliptic equations. Trans. Amer. Math. Soc. 333, 339-364 (1992)

14. Li, Y.: Asymptotic behavior of positive solutions of equation $\Delta u+K(x) u^{p}=0$ in $\mathbb{R}^{n}$. J. Differential Equations 95, 304-330 (1992)

15. Li, Y.: On the positive solutions of the Matukuma equation. Duke Math. J. 70, 575-589 (1993)

16. Li, Y., Ni, W.-M.: On conformal scalar curvature equations in $\mathbb{R}^{n}$. Duke Math. J. 57, 895-924 (1988) 
17. Li, Y., Ni, W.-M.: On the existence and symmetry properties of finite total mass solutions of the Matukuma equation, the Eddington equation and their generalizations. Arch. Rational Mech. Anal. 108, 175-194 (1989)

18. Li, Y., Ni, W.-M.: On the asymptotic behavior and radial symmetry of positive solutions of semilinear elliptic equations in $\mathbb{R}^{n}$, Part I. Asymptotic Behavior, Arch. Rational Mech. Anal. 118, 195-222 (1992)

19. Li, Y., Ni, W.-M.: On the asymptotic behavior and radial symmetry of positive solutions of semilinear elliptic equations in $\mathbb{R}^{n}$, Part II. Radial Symmetry, Arch. Rational Mech. Anal. 118, 223-244 (1992)

20. Li, Y., Santanilla, J.: Existence and nonexistence of positive singular solutions for semilinear elliptic problems with applications. Differential Integral Equations 8, 1369-1383 (1995)

21. Liu, Y., Li, Y., Deng, Y.B.: Separation property of solutions for a semilinear elliptic equation. J. Differential Equations 20, 381-406 (2000)

22. Matukuma, T.: Sur la dynamique des amas globulaires stellaires. Proc. Tmp. Acad. 6, 133-136 (1930)

23. Ni, W.-M.: Uniqueness, Nonuniqueness and related questions of nonlinear elliptic and parabolic equations. Proc. Symp. Pure Appl. Math. 45, Amer. Math. Soc., 229-241 (1986)

24. Ni, W.-M., Yotsutani, S.: Semilinear elliptic equations of Matukuma-type and related topics. Japan J. Appl. Math. 5, 1-32 (1988)

25. Rein, G.: Static shells for the Vlasov-Poisson and Vlasov-Einstein systems. Indiana Univ. Math. J. 48, 335-346 (1999)

26. Rein, G.: Flat steady states in stellar dynamics - Existence and stability. Comm. Math. Phys. 205, 229-247 (1999)

27. Rein, G.: Stationary and static stellar dynamic models with axial symmetry. Nonlinear analysis; Theory, Methods and Applications 41, 313-344 (2000)

28. Rein, G.: Static solutions of the spherically symmetric Vlasov - Einstein system. Math. Proc. Comb. Phil. Soc. 115, 559-570 (1994)

29. Rein, G.: Static shells for the Vlasov-Poisson and Vlasov-Einstein systems. Indiana University Math. J. 48, 335-346 (1999)

30. Rein, G.: Collisionless Kinetic Equations from Astrophysics - The Vlasov Poisson System in: Handbook of Differential Equations, Evolutionary Equations, Vol. 3, Chapter 5, 383-476 (C.M. Dafermos and E. Feireisl ed.), Elsevier 2007.

31. Rein, G., Rendall, A.D.: Smooth static solutions of the spherically symmetric Vlasov - Einstein system. Ann. de l'Institut H. Poincaré, Physique Théorique 59, 383-397 (1993)

32. Rein, G., Rendall, A.D.: Compact support of spherically symmetric equilibria in non-relativistic and relativistic galactic dynamics. Math. Proc. Camb. Phil. Soc. 128, 363-380 (2000)

33. Rein, G., Rendall, A.D., Schaeffer, J.: A regularity theorem for the spherically symmetric Vlasov-Einstein system. Comm. Math. Phys. 168, 467-478 (1995)

34. Thieme, H.R.: Asymptotically autonomous differential equations in the plane. Rocky Mountain J. Math. 24, 351-380 (1994)

35. Yanagida, E.: Uniqueness of positive radial solutions of $\triangle u+g(r) u+h(r) u^{p}=$ 0 in $\mathbb{R}^{n}$. Arch. Rational Mech. Anal. 115, 257-274 (1991)

36. Yanagida, E.: Structure of positive radial solutions of Matukuma's equation. Japan J. Indust. Appl. Math. 8, no. 1, 165-173 (1991)

37. Yanagida, E., Yotsutani, S.: Global Structure of Positive Solutions to Equations of Matukuma Type. Arch. Rational Mech. Anal. 134, 199-226 (1996)

38. Yanagida, E.: Extinction and blowup of positive radial solutions for a semilinear elliptic equation. Nonlinear Anal. 39, 365-377 (2000)

39. Wong, J.S.W.: On the generalized Emden-Fowler equation. SIAM Rev. 17, 339-360 (1975) 


\author{
Jürgen Batt \\ Mathematisches Institut \\ der Universität München \\ Theresienstr. 39, \\ D 80333 München, Germany \\ e-mail: batt@mathematik.uni-muenchen.de \\ Yi Li \\ Department of Mathematics \\ University of Iowa \\ Iowa City, Iowa 52242, USA \\ and \\ Department of Mathematics \\ Xian Jiaotong University \\ Xian, Shannxi, China \\ e-mail: yi-li@uiowa.edu
}

Keywords and phrases: Matukuma equation, radial solutions, semilinear elliptic equations, Emden-Fowler equation, asymptotically autonomous systems, Volterra systems, Vlasov-Poisson system, stationary stellar dynamic models 